\title{
Tsunami Coastal Hazard Along the Us East Coast From Coseismic Sources in the açores Convergence Zone and the Caribbean Arc Areas
}

Stephan Toni Grilli ( $\nabla$ grilli@egr.uri.edu )

University of Rhode Island https://orcid.org/0000-0001-8615-4585

Maryam Mohammadpour

University of Rhode Island

Lauren Schambach

University of Rhode Island

Annette Grilli

University of Rhode Island

\section{Research Article}

Keywords: Tsunami hazard assessment, Tsunami propagation, earthquakes, Boussinesq wave models

Posted Date: April 13th, 2021

DOI: https://doi.org/10.21203/rs.3.rs-404526/v1

License: (c) (i) This work is licensed under a Creative Commons Attribution 4.0 International License.

Read Full License

Version of Record: A version of this preprint was published at Natural Hazards on November 15th, 2021. See the published version at https://doi.org/10.1007/s11069-021-05103-y. 


\title{
Tsunami coastal hazard along the US East Coast from coseismic sources in the Açores Convergence Zone and the Caribbean Arc areas
}

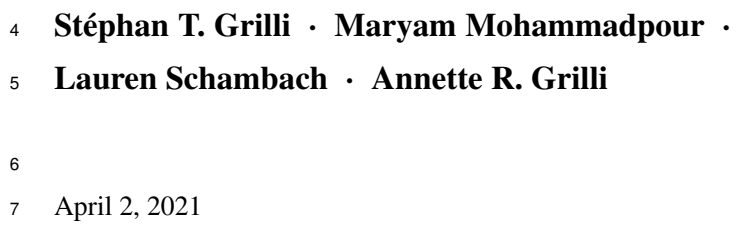

\begin{abstract}
We model the coastal hazard caused by tsunamis along the US East Coast (USEC) for far-field coseismic sources originated in the Açores Convergence Zone (ACZ), and the Puerto Rico Trench (PRT)/Caribbean Arc area. In earlier work, similar modeling was performed for probable maximum tsunamis (PMTs) resulting from coseismic, submarine mass failure and volcanic collapse sources in the Atlantic Ocean basin, based on which tsunami inundation maps were developed in high hazard areas of the USEC. Here, in preparation for a future Probabilistic Tsunami Hazard Analysis (PTHA), we model a collection of 18 coseismic sources with magnitude ranging from M8 to M9 and return periods estimated in the 100-2,000 year range. Most sources are hypothetical, based on the seismo-tectonic data known for the considered areas. However, the largest sources from the ACZ, which includes the region of the Madeira Tore Rise, are parameterized as repeats of the 1755 M8.6-9 (Lisbon) earthquake and tsunami using information from many studies published on this event, which is believed to have occurred east of the MTR. Many other large events have been doc-
\end{abstract}

S.T. Grilli

Department of Ocean Engineering, University of Rhode Island, Narragansett, RI, USA

E-mail: grilli@uri.edu

M. Mohammadpour

Department of Ocean Engineering, University of Rhode Island, Narragansett, RI, USA

E-mail: mmohammadpour@uri.edu

L. Schambach

Department of Ocean Engineering, University of Rhode Island, Narragansett, RI, USA

E-mail: 1schambach@uri.edu

\section{A.R. Grilli}

Department of Ocean Engineering, University of Rhode Island, Narragansett, RI, USA

E-mail: annette_grilli@uri.edu 
umented to have occurred in this area in the past 2,000 years. There have also been many large historical coseismic tsunamis in and near the Puerto Rico Trench (PRT) area, triggered by earthquakes with the largest in the past 225 years having an estimated M8.1 magnitude. In this area, coseismic sources are parameterized based on information from a 2019 USGS Powell Center expert, attended by the first author, and a collection of SIFT subfaults for the area (Gica et al., 2008).

For each source, regional tsunami hazard assessment is performed along the USEC at a coarse $450 \mathrm{~m}$ resolution by simulating tsunami propagation to the USEC with FUNWAVETVD (a nonlinear and dispersive (2D) Boussinesq model), in nested grids. Tsunami coastal hazard is represented by four metrics, computed along the $5 \mathrm{~m}$ isobath, which quantify inundation, navigation, structural, and evacuation hazards: (1) maximum surface elevation; (2) maximum current velocity; (3) maximum momentum force; and (4) tsunami arrival time. Overall, the first three factors are larger, the larger the source magnitude, and their alongshore variation shows similar patterns of higher and lower values, due to bathymetric control from the wide USEC shelf, causing similar wave refraction patterns of focusing/defocusing for each tsunami. The fourth factor differs mostly between sources from each area (ACZ and PRT), but less so among sources from the same area; its inverse is used as a measure of increased hazard associated with short warning/evacuation times. Finally, a new tsunami intensity index (TII) is computed, that attaches a score to each metric within 5 hazard intensity classes selected for each factor, reflecting low, medium low, medium, high and highest hazard, and is computed as a weighted average of these scores (weights can be selected to reinforce the effect of certain metrics). For each source, the TII provides an overall tsunami hazard intensity along the USEC coast that allows both a comparison among sources and a quantification of tsunami hazard as a function of the source return period. At the most impacted areas of the USEC (0.1 percentile), we find that tsunami hazard in the 100-500 year return period range is commensurate with that posed by category 3-5 tropical cyclones, taking into account the larger current velocities and forces caused by tsunami waves.

Based on results of this work, high-resolution inundation PTHA maps will be developed in the future, similar to the PMT maps, in areas identified to have higher tsunami hazard, using more levels of nested grids, to achieve a 10-30 m resolution along the coast.

Keywords Tsunami hazard assessment $\cdot$ Tsunami propagation · earthquakes · Boussinesq wave models 


\section{Introduction}

Since 2010, under the auspice of the US National Tsunami Hazard Mitigation Program (NTHMP; http://nthmp.tsunami.gov/index.html), the authors and colleagues have performed tsunami modeling work to develop high-resolution tsunami inundation maps for the US east coast (USEC), starting with the most critical or vulnerable areas, but with the goal to eventually to cover the entire coast. These so-called first generation maps were constructed as envelopes of maximum inundation caused by the most extreme near- and far-field tsunami sources in the Atlantic Ocean basin, a.k.a., Probable Maximum Tsunamis (PMTs), parameterized based on historical or hypothetical events [48]. In this first generation work, no return periods were considered for each source and probabilistic tsunami hazard analyses (PTHA) were left out for future work. Extreme tsunamigenic sources causing PMTs that can impact the USEC have been identified and modeled in past work [2,3,34,4,32,55, 17, 36, 8, 13, 23, 1, 19, 20, 24, 25, 21, 27, 57, 54, 49, 37, 45]. These sources were divided into three groups: 1) coseismic, 2) submarine mass failure (SMF), and 3) volcanic flank collapse. An overview of the global coastal hazard resulting from these sources, at the coarse regional scale, can be found in Schambach et al. [48].

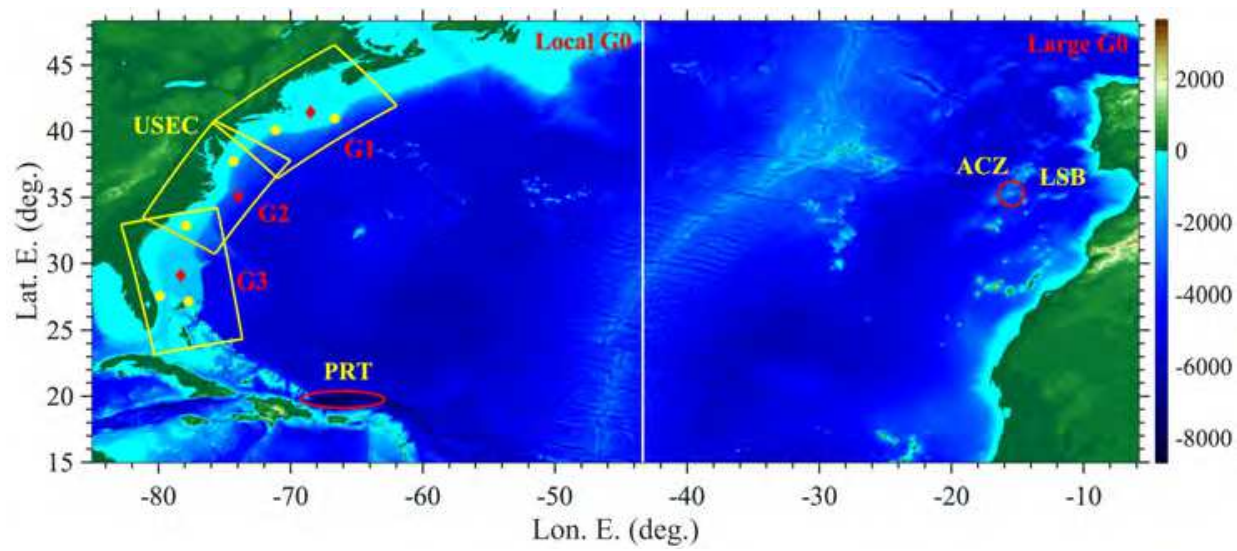

Fig. 1 Footprint and ETOPO1 bathymetry/topography (color scale in meter shows $<>0$ ) of FUNWAVE's 1 arc-min resolution grids in North Atlantic Ocean basin (Local/Large G0), with footprints of 3 regional 450 $\mathrm{m}$ nested shore-parallel Cartesian grids $(\mathrm{G} 1, \mathrm{G} 2$, G3; Table 4. Location are marked for the two areas of historical/hypothetical tsunami coseismic sources (red oval) considered, near the Açores Convergence Zone (ACZ), including Lisbon 1755 (LSB), and near and around the Puerto Rico Trench (PRT). The Madeira Torre Rise (MTR) is the shallower ridge located on the north of the ACZ circled area and the Horseshoe Plain is to the East of the ACZ and MTR. Yellow/red symbols within the regional grids mark locations of numerical wave gauge stations where time series of surface elevation are calculated in simulations for validating the one-way grid coupling (Table 2). 
The present work is part of the initial preparatory steps necessary to perform a complete PTHA for the USEC and create the next generation of probabilistic NTHMP inundation maps. PTHA, instead of only considering PMTs, requires combining simulation results for a collection of tsunami sources of different magnitudes and, hence, return periods for each type of sources. Here, we only consider far-field coseismic sources in the North Atlantic Ocean Basin (NAOB), which are the only seismic sources that can be sufficiently tsunamigenic to cause a significant tsunami hazard along the USEC, where near-field seismicity is quite moderate [55,57]. Additionally, coseismic sources, as a group, are believed to have lower return periods than the SMF and volcanic collapse sources in the NAOB [55, 57]. Hence, in its lower impact range, this work also provides a first comprehensive assessment of regional scale tsunami hazard for the entire USEC, for a range of return periods commensurate with those considered in risk analyses for coastal and ocean structures and infrastructures, for other natural hazard events such as tropical cyclones (e.g., 100 to 500 years).

We consider and model coseismic tsunamis generated by a collection of seismic sources located near and around (Fig. 11): (i) the Açores Convergence Zone (ACZ; Fig. 1] and see Fig. 18 in [57]), including the estimated location of the 1755 Lisbon (LSM) earthquake source, and (ii) the Puerto Rico Trench (PRT) and Caribbean arc area [33, 56, 28, 21]. Several large tsunamigenic earthquakes have occurred in these areas. In the ACZ/LSB area, which is located along the western segment of the Eurasia-Nubia Plate Boundary, between the Azores archipelago and the Strait of Gibraltar [7], some of these historical earthquakes have caused large transoceanic tsunamis, but the Lisbon 1755 earthquake, of estimated magnitude M8.69 [41,8], triggered the largest known historical tsunami. With 5-15 m high waves impacting the coast, this event caused extensive destruction and tens of thousands of fatalities, in the near-field in Lisbon and its area (in combination with seismicity and fires), while also reaching the coasts of Morocco, England, Newfoundland, Brazil, and the Antilles. In particular, after transoceanic propagation, the LSB 1755 tsunami still caused a few meters of inundation in the eastern Lesser Antilles [2,3,62,4,8,5,57,7]. Large coseismic tsunamis have also occurred in the PRT and Caribbean arc area [33,55,23,40], which a highly seismic area running parallel to the north shore of Hispaniola, Puerto Rico, and the north-eastern lesser Antilles [11,62,55,56, 23, 28,57] (see Fig. 11 for the observed distribution of earthquakes, in magnitude and depth). The PRT is the only subduction zone in the NAOB, in which the North America Plate subducts under the Caribbean Plate with a nearly E-W relative plate motion (i.e., largely left lateral strike slip; see large black arrows in Fig. 1) and only a small component of perpendicular convergence (3-6 mm/yr) [11 9]. Nevertheless, in: (i) 1842, a M8 earthquake in the western segments of the Septentrional fault (SF), which runs nearshore parallel to the north shore of Hispaniola, triggered a large tsunami that impacted in Haiti [12, 
16.21]; (ii) 1787, a M8.1 in the PRT triggered a moderate tsunami; and (iii) 1918, a M7.3 earthquakes in the Mona Passage, $15 \mathrm{~km}$ off the northwest coast or Puerto Rico, generated a tsunami that caused 116 fatalities and up to 6 m runup [40 35]. Since the Indian Ocean M9.2 earthquake and tsunami [22,30], we know that highly oblique subduction zones can generate devastating tsunamis if the earthquake rupture has a large thrust component. Although this is still controversial, some work has shown that a tsunamigenic M8.7-9 earthquake in the PRT would cause a devastating tsunami in the near-field and a significantly damaging event in the far-field, particularly along the upper USEC [32,23]. A recent meeting of experts at the USGS Powell Center (May 2019) reached the conclusion that such large PRT events were possible, however, the upper bound magnitude would likely require that fault segments in part of Hispaniola on the West and the Caribbean arc (up to Guadeloupe) on the East be also involved (this is further detailed later).

In this work, we parameterize and simulate tsunamis generated by coseismic sources sited in the two selected areas, ranging from magnitude M8 to M9. For the ACZ/LSB area, we will define sources sited at two different locations and having 2 different orientations (strike) of 15 and 345 deg. from North, besides different magnitude (M8-M9) and corresponding fault plate areas; this yields ten (10) different coseismic tsunami sources, four of these being the M9 PMT sources already considered in earlier NTHMP inundation mapping work [19,48]. Due the large uncertainty of sources in this area, each source is only represented by a single fault plane. As in earlier work considering PMTs [19], the two selected orientations are aimed at maximizing tsunami impact on the upper and lower USEC, respectively. Since the LSB/ACZ sources are quite distant from the USEC, tsunami propagation and refraction over the entire ocean width make details of the sources relatively less important [54], hence our earlier work that also considered additional orientations for the sources showed that these two selected strike angles were sufficient to reflect tsunami hazard along the USEC. We shall see that a repeat of the LSB M9 1755 event, particularly if it occurred in the ACZ, West of the Madeira Torre Rise (Fig. 1), a submarine ridge that somewhat diverted the transoceanic propagation of the historical event towards the USEC, would have the potential for causing high tsunami hazard along the entire USEC. In the PRT area, we only model hypothetical events, following the recommendations on fault plane segmentation made during the 2019 Powell Center meeting of expert, and consider eight (8) M8-M9 sources that combine 10 to 26 SIFT (Short-term Inundation Forecast for Tsunamis) subfaults [17], with for comparison one of these being the M9 PMT source already considered in earlier NTHMP inundation mapping work [23, 20, 48].

Tsunami simulations are performed for the 18 selected coseismic sources using the fully nonlinear and dispersive Boussinesq model FUNWAVE-TVD [61,50,31], by one-way coupling in a series of nested spherical or Cartesian coordinate grids. FUNWAVE has been 
validated for a collection of tsunami benchmarks [53,29], and used in the simulation of many historical [60, 22, 30, 51,24, $31,52,21,26,46,47]$ and hypothetical [23, $1,25,54,49,27$, 45] tsunami case studies. As we only aim at assessing the global coastal tsunami hazard caused by the selected sources along the USEC, we only use two levels of nested grids (Fig. 1): (i) two large scale spherical grids over the North Atlantic Ocean (or half of it), with a 1 arc-min resolution, and (ii) 3 smaller regional shore-parallel Cartesian grids overlapping along the USEC with a $450 \mathrm{~m}$ resolution. For each source, because of the coarse resolution of the coastal grids, we will assess tsunami hazard using several metrics, computed at a short distance from shore, along the $5 \mathrm{~m}$ isobath and will leave inundation and runup simulations out for future work using higher-resolution grids. Based on similar results obtained for a collection of PMTs selected in the North Atlantic Ocean [48], as part of NTHMP work, high-resolution inundation maps were computed for the envelope of all the PMT results in USEC areas that were deemed at higher hazard or being critical (see project webpage: https: / /www1.udel.edu/kirby/nthmp.html). To do so, additional levels of nested grids were used to achieve a 10-30 m resolution at the coast (see, for instance, [25]). Note, however, although complex coastal morphologies and highly developed areas will be under-resolved at a $450 \mathrm{~m}$ resolution, from Florida to Massachusetts, the USEC is essentially made of a series of sandy barrier beach and barrier-island whose coastal bathymetry is quite simple, and hence coarse grid results should be accurate up to a short distance from shore and reflection off the coast also be sufficiently accurately represented in the model. In their simulations of the impact of the 2004 Indian Ocean tsunami along the coasts of Thailand, using FUNWAVE in a $450 \mathrm{~m}$ resolution nested grid, Ioualalen et al. [30] showed a good agreement between the predicted and observed runups at 58 locations.

Based on results of the present work, obtained for eighteen coseismic sources of various magnitude (and hence return period), and similar results for other types of sources to be obtained in future work (i.e., for submarine landslides, volcanic flank collapse, and meteotsunamis), PTHA analyses will be conducted and probabilistic inundation maps, similar to the current first generation NTHMP maps based on PMTs, will be developed in future work in areas identified to have higher tsunami hazard. To more easily identify these areas, the coastal tsunami hazard simulated for each source considered here will be quantified by computing 4 hazard metrics along the $5 \mathrm{~m}$ isobath: (1) maximum surface elevation; (2) maximum current velocity; (3) maximum momentum force; and (4) tsunami arrival time. Overall, the first three factors are larger, the larger the source magnitude, and the fourth factor is expected to differ mostly between sources from each area (ACZ and PRT), but less among sources from the same area.

Finally, a tsunami hazard intensity index (TII) will be computed for each source, and overall, that attaches a score to selected classes of hazard metrics (here 5 classes for each 
metric), and provides an overall tsunami hazard intensity (or score) at a large number of save points along the $5 \mathrm{~m}$ isobath.Similar indices have been proposed in earlier work and shown to be usefulness in coastal tsunami hazard assessment, to discriminate between low, medium and high hazard coastal areas [42,10].

In the following, we first detail recent studies of seismic sources in the NOAB and our source selection in the two selected areas (ACZ and PRT). We then present the modeling methodology and results in terms of the various tsunami hazard metrics computed along the USEC and, based on these, we compute and discuss the TII values for each source.

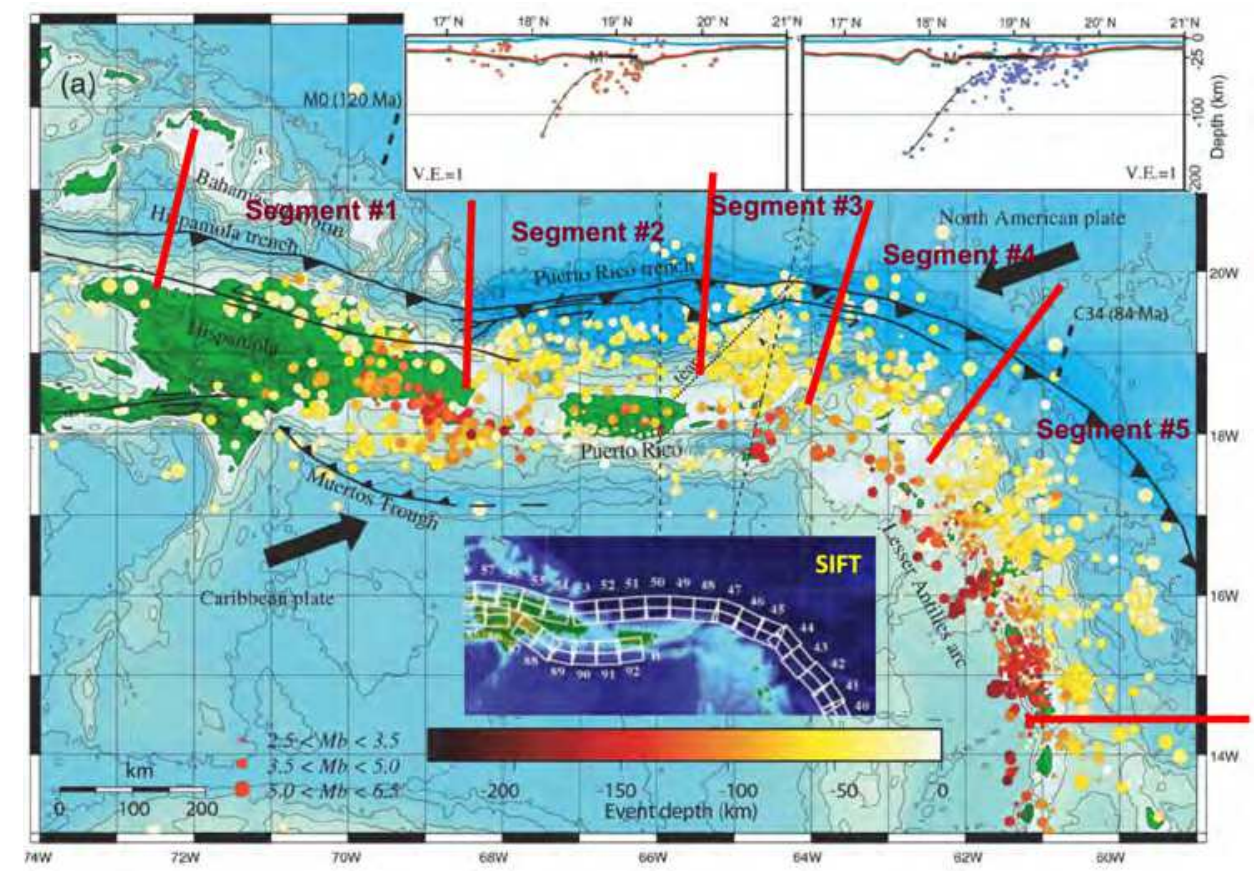

Fig. 2 Likeliest segmentation in 5 segments of the PRT/Caribbean arc, from west of Hispaniola to Guadeloupe in the eastern part, established at the May 2019 workshop of experts at the USGS Powell Center (see Fig. 1 for location). Insert shows footprints of SIFT subfaults [17] in the considered area (see Table 2 for parameter values). Large black arrows show the nearly E-W relative plate motion of the North America Plate subducting under the Caribbean Plate. 
2 Recent studies and selection of coseismic sources in the ACZ and PRT areas

\subsection{ACZ/LSB area}

By analyzing the historical sources with information about the occurrence of earthquakes and tsunamis in the region of Cape Saint Vincent-Gulf of Cádiz, Udias [59] reviewed large tsunamigenic earthquakes that occurred SW of Iberia before the 1755 Lisbon earthquake. Separating events that occurred before and after 500 A.D, the author concluded that the 1755 earthquake and tsunami was not an isolated event in SW Iberia and other similarly large events have occurred before the great Lisbon event (e.g., in 241/216 B.C., 881, 1356 and 1531), which is merely the most resent observed event in this category in the ACZ area (Fig. 1). Hence, there is a high likelihood for similarly large events to occur in the future in the ACZ/LSB area.

The exact location and parameters of the 1755 Lisbon earthquake, however, are still unknown and subject to debate. Various studies have placed its magnitude in the M8.6 to 9 range and its most likely location in the Horseshoe Fault to the East of the ACZ and Madeira Torre Rise (MTR; Fig. 1] [41,8]. MTR is an underwater ridge that likely caused westward propagating tsunami waves to be somewhat diverted from aiming at the USEC and, hence, offered some level of protection to the coast. Specifically, Baptista et al. [2,3] performed backward ray tracing and located the 1755 Lisbon source in the area between the Gorringe Bank and the southwestern end of the Portuguese coast. Based on field surveys and tsunami observations, Baptista et al. [4] later proposed a composite source in the Marquês de Pombal and Guadalquivir faults for the event. Barkan et al. [8] identified three potential coseismic tsunami sources for the historical Lisbon event located along three respective major faults, including: (i) the Gorringe Bank Fault (GBF); (ii) The Marques de Pombal Fault (MPF); (iii) The Gulf of Cadiz Fault (GCF). Based on a set of 16 potential tsunami source simulations performed around the areas of these three major faults, Barkan et al. inferred that the most likely source of the 1755 earthquake would have been located in the Horseshoe Plain thrust fault area (NW/SE strike). However, this fault may just be a paleo plate boundary [7]. Omira et al. [44] showed that a source in the Gorringe Bank would have radiated most of its energy towards the NE and Morocco, with a minor impact along the Gulf of Cadiz, and proposed two more potential locations for the source of the 1755 event, the: (iv) Horseshoe Fault and (v) Portimao Bank. They simulated tsunamis from the 5 potential areas and concluded that the most likely location was in Horseshoe Fault.

There have been a few recent tsunamigenic events in the ACZ area, which, although they did not generate large tsunamis, confirmed that large future earthquakes could potentially occur on both sides of the ASZ/MTR area. Hence, in comprehensive tsunami hazard 
assessment studies, both of these locations must be considered for siting such future events. Specifically, in 1941 a M8.3 strike-slip earthquake occurred in the Gloria fault, northwest of the ACZ. A small tsunami was registered at the tide stations of Cascais, Lagos, Portugal, Morocco, Madeira, Azores (and in the UK), with a maximum observed height of 0.45 m (peak to peak) at Casablanca in Morocco. In 1969, a M7.9 earthquake occurred in the Horseshoe Plain, directly east of the ACZ, which created a small tsunami with a maximum height of $0.06 \mathrm{~m}$ observed on the Portuguese coast in Lagos. And, in 1975, a M7.9 earthquake occurred south of the Gloria Fault, within the ACZ and southeast of the MTR [6.7] (Fig.11, which generated a tsunami recorded at a set of coastal tide gauges, with a measured amplitude of up to $0.3 \mathrm{~m}$ in Lagos.

As part of earlier NTHMP work, Grilli and Grilli [19] designed extreme M9 PMTs originated in the ACZ area, as repeats of the Lisbon 1755 event, based on the conclusions of Barkan et al.'s [8] study. To cover the range of uncertainty on source location and parameters, they modeled twelve sources of M9 magnitude sited at various locations in the ACZ, with parameters selected based on earlier published work. The strike angle, in particular, which strongly affects the tsunami directionality, was varied to cause maximum impact on various sections of the USEC. Among those sources, as expected, they found that maximum impact on the USEC, with a 1-2 m flow depth at the coast, was created by sources sited in the MTR, which is the westernmost potential site for the historical earthquake; arrival time on the USEC was between 8 and $12 \mathrm{~h}$, depending on the location. However, should a large coseismic source occur west of the MTR, it would cause an even larger impact on the USEC. Hence for extreme tsunami hazard assessment, such source locations should also be considered for future events.

Accordingly, in our recent work modeling coastal hazard caused by PMTs along the USEC as part of NTHMP [48], we considered four (4) so-called Lisbon (LSB) M9 PMTs, sited at locations both west of the MTR (M9.0-MTR1 and M9.0-MTR2) and east of the MTR (M9.0-HSP1 and M9.0-HSP2) in the Horseshoe Plain, and with strike angles of 15 or $345 \mathrm{deg}$. In combination, these sources should maximize the tsunami impact from extreme PMTs originating in this area along the entire USEC. In the absence of more detailed data, each source was made of a single fault plane, with parameters based on Barkan et al. [8] and Grilli and Grilli [19] listed in Table 1] Here, in addition to these extreme sources, to prepare for future PTHA work along the USEC, we design and parameterize 6 additional tsunamigenic coseismic sources with 3 smaller magnitudes, M8, 8.3, and 8.7, and for each the 2 strike angles, whose parameters are also listed in Table 1 All 10 sources are assumed to be similarly shallow to maximize tsunami generation, with a depth below seafloor $d=5$ $\mathrm{km}$ at their highest point, and sited at the same location west of the MTR to maximize their impact on the USEC, except for M9-HSP1 and M9-HSP2, which are sited in the horseshoe 
plain at the likeliest location for the 1755 event. While the M9 sources have a fault plane of length $L=317 \mathrm{~km}$ by width $W=126 \mathrm{~km}$ [8], the smaller magnitude sources have reduced dimensions derived based on the various references listed before. For each coseismic source, magnitudes $\mathrm{M}$ and fault slip $S$ are related by the formulas,

$$
M_{o}=\mu L W S
$$

where $M_{o}$ is the moment magnitude, $\mu=40 \mathrm{GPa}$ the material constant (i.e., Coulomb/shear modulus), and the magnitude is defined as,

$$
\mathrm{M}=\frac{2}{3} \log M_{o}-6
$$

For each source, using the fault plane parameters and location listed in Table 11. Okada's method [43] will be used to compute the initial surface elevation used to initialize the tsunami propagation model (see details later).

Although historical data is limited to accurately attach a return period to each of these selected ACZ/LSB events, the average recurrence of large LSB 1755 type events appears to be on the order of 500 years [59] (4-5 medium-large events in 2,000 years). Hence, based on the corresponding slip and fault area, one could infer that M8, M8.3, and M8.7 events would have a return periods of 70, 140, and 250 years with a large uncertainty. These return periods are listed in Table 1 for information, as probabilistic considerations will be addressed in future PTHA work.

\subsection{PRT and Caribbean Arc area}

While there have been many studies regarding the seismo-tectonic context and past nearfield tsunamis that have affected the PRT and Caribbean arc area [11,33,62, 55, 28, 57, 40] (see Fig. 11, until the 2019 Powell Center meeting of experts, no comprehensive study had considered the largest tsunamigenic coseismic sources that could affect the USEC, except perhaps work performed for the US nuclear regulatory commission [55,56].

Hence, when tsunami hazard assessment studies were initialed for the USEC, in the absence of specific guidance, Knight [32] first proposed a M9.1 PRT source as an extreme PMT, which encompassed the $600 \mathrm{~km}$ long deepest part of the PRT that runs parallels to the Puerto Rico North shore; this source was parameterized by a single fault plane. Using this and another similar source with a smaller M8.7 magnitude, Grilli et al. [23] modeled both the near-field tsunami impact on Puerto Rico and the far-field impact on the USEC. Based on earlier work [15,40,62], they assumed a predominantly (lateral) strike-slip motion of the Caribbean plate at $20 \mathrm{~mm}$ per year with respect to the North American Plate, in the ENE direction, at a 10-20 degree angle with respect to the PRT axis. Analyzing the historical 


\begin{tabular}{|c||c|c|c|c|c|c|c|c|}
\hline $\begin{array}{c}\text { Source } \\
\text { (ACZ) }\end{array}$ & $\begin{array}{c}\text { Lat. N. } \\
\text { (deg.) }\end{array}$ & $\begin{array}{c}\text { Lon. E. } \\
(\text { deg.) }\end{array}$ & $\begin{array}{c}\text { Strike } \\
\theta(\mathrm{deg} .)\end{array}$ & $\begin{array}{c}\text { Length } \\
L(\mathrm{~km})\end{array}$ & $\begin{array}{c}\text { Width } \\
W(\mathrm{~km})\end{array}$ & $\begin{array}{c}\text { Slip } \\
S(\mathrm{~m})\end{array}$ & $\begin{array}{c}\mathrm{M} \\
\sim T_{r} \\
\text { (year) }\end{array}$ \\
\hline \hline M8.0-MTR1 & 36.748 & -15.929 & 15 & 150 & 60 & 2.87 & 8.0 & 70 \\
M8.0-MTR2 & 36.748 & -15.929 & 345 & 150 & 60 & 2.87 & 8.0 & 70 \\
M8.3-MTR1 & 36.748 & -15.929 & 15 & 175 & 70 & 5.95 & 8.3 & 140 \\
M8.3-MTR2 & 36.748 & -15.929 & 345 & 175 & 70 & 5.95 & 8.3 & 140 \\
M8.7-MTR1 & 36.748 & -15.929 & 15 & 200 & 80 & 18.14 & 8.7 & 250 \\
M8.7-MTR2 & 36.748 & -15.929 & 345 & 200 & 80 & 18.14 & 8.7 & 250 \\
M9.0-MTR1 & 36.748 & -15.929 & 15 & 317 & 126 & 20 & 9.0 & 500 \\
M9.0-MTR2 & 36.748 & -15.929 & 345 & 317 & 126 & 20 & 9.0 & 500 \\
M9.0-HSP1 & 36.042 & -10.753 & 15 & 317 & 126 & 20 & 9.0 & 500 \\
M9.0-HSP2 & 36.042 & -10.753 & 345 & 317 & 126 & 20 & 9.0 & 500 \\
\hline
\end{tabular}

Table 1 Parameters of coseismic sources modeled as a single fault plane in the ASZ area (Fig. 1), to be used in Okada's method [43] to define a tsunami source. All sources are shallow, with a depth $d=5 \mathrm{~km}$ at the mid-fault highest point, and all fault planes are dipping at $\delta=40 \mathrm{deg}$., with the rake being $\gamma=90 \mathrm{deg}$. Estimates of return period $T_{r}$ for each events are provided for information only and have a large uncertainty. The M9 sources are Lisbon 1755 proxy sources based on Barkan et al. [8] and Grilli and Grilli [19], while the M8, 8.3 and 8,7 are smaller magnitude sources modeled to prepare for future PTHA along the USEC. Except for M9-HSP1 and M9-HSP2, which are sited in the horseshoe plain at the likeliest location for the 1755 event, all sources are centered at the same location west of the MTR (Fig. 1), to maximize impact on the USEC. Magnitudes and slip are based on a Coulomb modulus $\mu=40 \mathrm{GPa}$. Fig. 4 shows the initial elevation computed for the 4 largest sources; other sources have similar patterns of elevations and depression, but smaller amplitude initial surface elevations.

earthquakes and the tsunamigenic events among those, they noted that 12 earthquakes of at least a M7 magnitude had occurred in and near the PRT area in the past 500 years [14]. Among those, two had a M8.1 magnitude, and three generated a tsunami with a 5-7 $\mathrm{m}$ on Puerto Rico. Combining these observations with the plate convergence rates, they estimated that a M7.5-8.1 event in the PRT would have an 80 year return period, a M8.7 event at least a 200 year return period, and a M9 event at least a 600 year return period. In view of more recent work on the segmentation and fault locking in the area (see below), it is likely that these return periods were under-estimated.

Simulating Knight's extreme M9 event, Grilli et al. [23] showed that the generated tsunami could cause up to 2-3 m runup along the USEC and arrival times would be between 2.5 and $6 \mathrm{~h}$, depending on the location. In our initial NTHMP work, in light of new data provided on subfault parameters in the PRT/Caribbean arc area as part of NOAA's SIFT dataset [17], Grilli et al. [20] designed and modeled a different M9 PMT source, which approximately covered the same area of the PRT as Knight's [32] source, i.e., $600 \mathrm{~km}$ long by $150 \mathrm{~km}$ wide, but was parameterized as 6 by 2 (i.e., 12) individual SIFT subfaults, each 100 long by $50 \mathrm{~km}$ wide (in the oblique fault plane), which better represented the convex 
geometry of the PRT. Although this was an extreme scenario, particularly for Puerto Rico, their work on the 2004 Indian Ocean M9.2 earthquake [22,30] and devastating tsunami had convinced these authors, as others [55,56], that a large megathrust event could occur in the PRT because of the similarity between the geometry (both trenches are arched) and plate dynamics of the Puerto Rico and Sumatra-Andaman trenches. This was also supported by their work on the Tohoku 2011 M9 earthquake and tsunami, that occurred in the similar size Japan trench [24,52]. Most recently, simulations of tsunami hazard caused by PMTs on the USEC were performed at higher resolution using as one of the sources that of Grilli et al. [20], which confirmed the range of runup and arrival time predicted earlier [48].

In the present work, we needed to design and model a collection of hypothetical, but realistic, coseismic tsunami sources of various magnitude (and return periods) in the complex PRT/Caribbean arc area, that would significantly impact the USEC. As indicated before, a meeting of experts was organized in May 2019 at the USGS Powell Center, attended by the lead author, whose agenda was devoted in large part to establishing a so-called logic tree for coseismic sources in the PRT and Caribbean arc area that could cause tsunamis affecting the USEC. Such a tree visualizes the various sources and their parameter range, while attaching a probability to each branch off the tree. The workshop approach was based on the Delphi method, which is a process used to arrive at a group opinion by surveying the experts attending the venue. Through several rounds of questionnaires, the responses provided by the experts on various source parameters were transformed into tables of probabilities for classes of parameter values.

The consensus of the experts at the meeting was that a first step was, by considering existing seismo-tectonic knowledge in the area, to establish a segmentation of the entire Caribbean arc into realistic segments, most likely to fail either separately or together in clusters, and to estimate their parameters. Sources of various magnitude could then be designed by combining those segments. Additionally, in the PRT and on both sides of it, there was large uncertainty on the level of locking and the magnitude of the fault-normal convergence rate that would most contribute to causing large tsunamigenic earthquakes [9], whose range needed to be estimated. Figure 2 shows the likeliest segmentation established during the meeting, which is composed of 5 segments, of the faults encompassing subduction zones in the Hispaniola trench on the west (Segment \#1), the PRT in the middle (Segments \#2,3), and in the northern Lesser Antilles arc (Segments \#4,5). Each of these segments approximately overlaps with subfaults defined in the SIFT dataset [17] (see insert in Fig. 2). For each SIFT subfault, of dimension $L=100$ by $W=50 \mathrm{~km}$ in the trench-parallel and trench-normal directions, respectively, the dataset provides the strike and dip angles, with the rake angle assumed as $\gamma=90 \mathrm{deg}$. in all cases (to maximize the seafloor deformation), and the lat-lon coordinates of the fault centroid and depth $d$ of the fault highest point. Table 2 provides 


\begin{tabular}{|c|c|c|c|c|c|c|}
\hline $\begin{array}{c}\text { Segment } \\
\#\end{array}$ & $\begin{array}{c}\text { SIFT } \\
\text { subfault }\end{array}$ & $\begin{array}{l}\text { Lon. E. } \\
\text { (deg.) }\end{array}$ & $\begin{array}{l}\text { Lat. N. } \\
\text { (deg.) }\end{array}$ & $\begin{array}{l}\text { Depth } \\
d(\mathrm{~km})\end{array}$ & $\begin{array}{c}\text { Strike } \\
\theta \text { (deg.) }\end{array}$ & $\begin{array}{c}\text { Dip } \\
\delta \text { (deg.) }\end{array}$ \\
\hline 1 & atsza57 & -72.3535 & 19.4838 & 22.10 & 94.20 & 20.00 \\
\hline 1 & atszb57 & -72.3206 & 19.9047 & 5.00 & 94.20 & 20.00 \\
\hline 1 & atsza56 & -71.5368 & 19.3853 & 22.10 & 102.64 & 20.00 \\
\hline 1 & atszb56 & -71.4386 & 19.7971 & 5.00 & 102.64 & 20.00 \\
\hline 1 & atsza55 & -70.7045 & 19.1376 & 22.10 & 108.19 & 20.00 \\
\hline 1 & atszb55 & -70.5647 & 19.5386 & 5.00 & 108.19 & 20.00 \\
\hline 1 & atsza54 & -69.6740 & 18.8841 & 22.10 & 101.54 & 20.00 \\
\hline 1 & atszb54 & -69.5846 & 19.2976 & 5.00 & 101.54 & 20.00 \\
\hline 2 & atsza53 & -68.4547 & 18.7853 & 22.10 & 83.64 & 20.00 \\
\hline 2 & atszb53 & -68.5042 & 19.2048 & 5.00 & 83.64 & 20.00 \\
\hline 2 & atsza52 & -67.5412 & 18.8738 & 22.10 & 85.87 & 20.00 \\
\hline 2 & atszb52 & -67.5734 & 19.2948 & 5.00 & 85.87 & 20.00 \\
\hline 2 & atsza51 & -66.5742 & 18.9484 & 22.10 & 84.98 & 20.00 \\
\hline 2 & atszb51 & -66.6133 & 19.3688 & 5.00 & 84.98 & 20.00 \\
\hline 2 & atsza50 & -65.6921 & 18.9848 & 22.10 & 89.59 & 20.00 \\
\hline 2 & atszb50 & -65.6953 & 19.4069 & 5.00 & 89.59 & 20.00 \\
\hline 3 & atsza49 & -64.8153 & 18.9650 & 22.10 & 94.34 & 20.00 \\
\hline 3 & atszb49 & -64.7814 & 19.3859 & 5.00 & 94.34 & 20.00 \\
\hline 3 & atsza 48 & -63.8800 & 18.8870 & 22.10 & 95.37 & 20.00 \\
\hline 3 & atszb48 & -63.8382 & 19.3072 & 5.00 & 95.37 & 20.00 \\
\hline 4 & atsza47 & -63.1649 & 18.7844 & 22.10 & 110.46 & 20.00 \\
\hline 4 & atszb47 & -63.0087 & 19.1798 & 5.00 & 110.46 & 20.00 \\
\hline 4 & atsza46 & -62.4217 & 18.4149 & 17.94 & 117.86 & 15.00 \\
\hline 4 & atszb46 & -62.2075 & 18.7985 & 5.00 & 117.86 & 15.00 \\
\hline 4 & atsza45 & -61.5491 & 18.0566 & 17.94 & 112.84 & 15.00 \\
\hline 4 & atszb45 & -61.3716 & 18.4564 & 5.00 & 112.84 & 15.00 \\
\hline 5 & atsza44 & -61.1559 & 17.8560 & 17.94 & 141.07 & 15.00 \\
\hline 5 & atszb44 & -60.8008 & 18.1286 & 5.00 & 141.07 & 15.00 \\
\hline 5 & atsza43 & -60.5996 & 17.0903 & 17.94 & 138.71 & 15.00 \\
\hline 5 & atszb43 & -60.2580 & 17.3766 & 5.00 & 138.71 & 15.00 \\
\hline 5 & atsza42 & -59.9029 & 16.4535 & 17.94 & 136.99 & 15.00 \\
\hline 5 & atszb42 & -59.5716 & 16.7494 & 5.00 & 136.99 & 15.00 \\
\hline
\end{tabular}

Table 2 SIFT subfault and their parameters from Gica et al. [17, for each segment selected for defining coseismic tsunami sources in the PRT/Caribbean arc area (Figs. 1 and 1 using the Okada [43] method. In addition, each subfault has a (horizontal) length $L=100 \mathrm{~km}$, width $W=50 \mathrm{~km}$, and rake $\gamma=90 \mathrm{deg}$. The material constant (shear modulus) used is $\mu=40 \mathrm{GPa}$, as recommended in [17]. these parameters for all the SIFT subfaults overlapping with Segments \#1 to 5 from Fig. 2 , with the subfault locations defined in the figure inset.

The second step considered by the experts was defining the likeliest grouping of individual segments that would fail together in a single event, thus creating sources of larger 
magnitude, as well as the associated magnitude for each such event and their likelihood (i.e., probability). Although the final proceedings of the workshop are still pending, based on draft material from the workshop, Table 2.2 lists 7 new sources in the PRT/Caribbean arc area, with magnitudes M8.3, M8.7, and M9.0, that regroup 2 to 4 segments from Fig. 2; an additional eighth source M9.0-PRT3 is the extreme PMT source used in earlier work to date [20 48]. Based on the SIFT subfault dimensions and their number used for each source listed in Table 3, an associated average slip $S$ was computed for using Eqs. 1 and 2 assuming $\mu=40 \mathrm{GPa}$ as in the SIFT study. For each source, using the fault plane parameters and location listed in Table 3, Okada's method [43] will be used to compute the initial surface elevation used to initialize the tsunami propagation model (see details later).

If the maximum convergence rate of $\sim 20 \mathrm{~mm} / \mathrm{y}$ (i.e., $1 \mathrm{~m}$ of slip in 50 years) in the area contributed to the listed slip values, the return period for each source would be $\sim 75,185$, and 355 years, respectively. However, the relative subduction between the Caribbean and North-American plates is highly oblique (10-20 deg.) on the western side of the considered area and less so in the lesser Antilles (Fig. 2). Accumulated fault slip must be multiplied by the sine of the convergence angle; hence, assuming an average of $20 \mathrm{deg}$. for the entire area yields a factor of one-third or so and approximate return periods on the order of 250, 550, and 1,000 years for each source magnitude, respectively, which is consistent with estimates made during the Powell workshop; applying the same considerations to the M9.0-PRT3 source, we find an approximate return period of years of 2,000 years (i.e., about 3 times the earlier estimate that did not consider the oblique subduction [23]). The estimated return periods are listed for each source in Table 2.2 however, detailed probabilistic considerations will be left out for future PTHA work.

\section{Methodology}

\subsection{Tsunami propagation model}

For each of the selected coseismic sources, tsunami propagation to the USEC is modeled using the nonlinear and dispersive two-dimensional (2D) Boussinesq long wave model (BM) FUNWAVE [61], in a series of nested grids of increasing resolution, by a one-way coupling method. We use FUNWAVE-TVD V.3, the newer implementation of FUNWAVE, which is fully nonlinear in Cartesian grids [50] and weakly nonlinear in spherical grids [31]. The model was efficiently parallelized for use on a shared memory cluster, which allows using large grids. FUNWAVE and then FUNWAVE-TVD are open source codes that have been widely used to simulate tsunami case studies [22, 30, 51, 23, 1, 52, 24 , 31, 54, 25, 21, 27, 26. 46 47]. As discussed in the introduction, since 2010 as part of the NTHMP work (see, 


\begin{tabular}{|c||c|c|c|c|c|}
\hline $\begin{array}{c}\text { Source } \\
\text { (PRT) }\end{array}$ & $\begin{array}{c}\text { Segments } \\
\text { number }\end{array}$ & $\begin{array}{c}\text { Nb. } \\
\text { SIFT }\end{array}$ & $\begin{array}{c}\text { Slip } \\
S(\mathrm{~m})\end{array}$ & $\mathrm{M}$ & $\begin{array}{c}\sim T_{r} \\
\text { (year) }\end{array}$ \\
\hline \hline M8.3-PRT1 & $\# 2-3$ & 12 & 1.3 & 8.3 & 250 \\
M8.3-PRT2 & $\# 3-4$ & 10 & 1.6 & 8.3 & 250 \\
M8.3-PRT3 & $\# 4-5$ & 12 & 1.3 & 8.3 & 250 \\
M8.7-PRT1 & $\# 2-4$ & 18 & 3.5 & 8.7 & 550 \\
M8.7-PRT2 & $\# 3-5$ & 16 & 3.9 & 8.7 & 550 \\
M9.0-PRT1 & $\# 1-4$ & 26 & 6.8 & 9.0 & 1,000 \\
M9.0-PRT2 & $\# 2-5$ & 24 & 7.4 & 9.0 & 1,000 \\
M9.0-PRT3 & $\# 2-3$ & 12 & 14.8 & 9.0 & 2,000 \\
\hline
\end{tabular}

Table 3 Definition in terms of segmentation established at the USGS 2019 Powell Center workshop, of coseismic sources modeled as multiple SIFT subfault planes in the PRT/Caribbean arc area (Fig. 1), to be used in Okada's method [43] to define a tsunami source (Fig. 1). Magnitude and slip (based on a Coulomb modulus $\mu=40 \mathrm{GPa}$ ) are given in the table and other parameters of SIFT sources used in each segment are given in Table 2 Estimates of return period $T_{r}$ for each events are provided for information only and have a large uncertainty. The collection of sources is modeled to prepare for future PTHA along the USEC. Fig. 5 shows the initial surface elevations computed for these sources. Note that source M9.0-PRT3 is the extreme PMT source used in earlier work [23,20 48].

http://chinacat.coastal.udel.edu/nthmp.html; e.g., [1, 19,20,25|,27,54,45]), the authors have used FUNWAVE and related methodology to simulate PMTs and compute tsunami inundation maps along the USEC. The same model and approach were also used to perform several other tsunami hazard assessment studies of coastal nuclear power plants in the U.S. Both spherical and Cartesian versions of FUNWAVE-TVD were validated through benchmarking and approved for NTHMP work [53,29,38].

As they include frequency dispersion effects, BMs simulate more complete physics than models based on Nonlinear Shallow Water Equations (NSWE), which until recently were traditionally used to simulate coseismic tsunami propagation. Dispersive models are necessary to accurately simulate landslide tsunamis [18, 45], which are typically made of shorter and hence more dispersive waves than coseismic tsunamis [60]. However, dispersion is also important for modeling the propagation of coseismic tsunamis over long distances [31], and their coastal impact when using high resolution grids, since undular bores can be generated nearshore near the crest of incident long waves [39,45]. The importance of dispersion for modeling tsunami propagation was confirmed by running FUNWAVE in both BM and NSWE modes, e.g., for the 1998 Papua New Guinea landslide tsunami [51], for the 2004 Indian Ocean [30] and for the 2011 Tohoku coseismic tsunamis [31], and for the 2018 Palu tsunami [47].

Along the shore, FUNWAVE has an accurate moving shoreline algorithm that identifies wet and dry grid cells, allowing to dynamically compute tsunami inundation and runup. Bot- 


\begin{tabular}{|c||c|c|c|c|c|c|c|c|}
\hline Grid/ & $\begin{array}{c}\text { Lat. N. } \\
(\text { SW/CT }) \\
\text { Type }\end{array}$ & $\begin{array}{c}\text { Lon. E. } \\
(\mathrm{SW} / \mathrm{CT}) \\
(\mathrm{deg})\end{array}$ & $\begin{array}{c}\text { Lat. N. } \\
(\mathrm{NE})\end{array}$ & $\begin{array}{c}\text { Lon. E. } \\
\text { (deg.) }\end{array}$ & $\begin{array}{c}\text { Res. } \\
(\mathrm{deg})\end{array}$ & $N_{x}$ & $N_{y}$ & $\begin{array}{c}\text { Angle } \\
\text { (clock.) } \\
\text { (deg.) }\end{array}$ \\
\hline \hline Large G0/S & 15 & -85 & 48.32 & -5.85 & $1 \mathrm{~min}$ & 4,750 & 2,000 & 0 \\
Local G0/S & 15 & -85 & 48.32 & -43.35 & $1 \mathrm{~min}$ & 2,500 & 2,000 & 0 \\
G1/C & 41.500 & $-69.00)$ & & & $450 \mathrm{~m}$ & 2,200 & 1,416 & 39.66 \\
G2/C & 35.750 & -75.75 & & & $450 \mathrm{~m}$ & 2,100 & 1,332 & 57.90 \\
G3/C & 28.705 & -78.05 & & & $450 \mathrm{~m}$ & 2,472 & 1,536 & 101.2 \\
\hline
\end{tabular}

Table 4 Parameters of model grids used in FUNWAVE to compute the propagation of far-field coseismic sources from the ACZ/LSB and PRT areas (Figs. 113. "Res." refers to the resolution of Spherical (S) or Cartesian (C) type grids and $N_{x}$ and $N_{y}$ indicate the number of grid cells in each direction. Letters in parenthesis indicate whether coordinates are for: (SW) Southwest corner, (NW) corner, or (CT) center of grid.

\begin{tabular}{|c||c|c|}
\hline Save Point & Lat. N. (deg.) & Lon. E. (deg.) \\
\hline \hline P-G1 & 41.430 & -68.480 \\
P-G2 & 35.050 & -73.950 \\
P-G3 & 29.070 & -78.300 \\
P1 & 40.954 & -66.632 \\
P2 & 40.084 & -71.143 \\
P3 & 37.709 & -74.309 \\
P4 & 32.842 & -77.910 \\
P5 & 27.579 & -79.888 \\
P6 & 27.183 & -77.711 \\
\hline
\end{tabular}

Table 5 Nested grid save points P-G1 to G3 (red diamonds in Fig. 1 used to compare time series of surface elevation with those computed in grid G0. Numerical wave gauges P1 to P6 where additional time series are computed in grids G1, G2 and G3 (yellow dots symbols in Fig. 1); P1 to P5 are located along the $200 \mathrm{~m}$ isobath (from north to south) and P6 is in 1,000 m depth (southernmost point north of the Great Bahama Banks off of Florida).

tom friction is modeled by a quadratic law, with a constant friction coefficient; in the absence of more specific data, we used the standard value $C_{d}=0.0025$, which corresponds to coarse sand and is conservative as far as tsunami runup and inundation. Earlier work indicates that tsunami propagation results are not very sensitive to the friction coefficient values in deeper water, but that they are affected in shallower water; in particular, the modeled wave height decay over a wide shallow shelf strongly depends on bottom friction [54]. Finally, dissipation from breaking waves is modeled using a front tracking (TVD) algorithm and switching to NSWEs in grid cells where breaking is detected (based on a breaking criterion). Earlier work has shown that numerical dissipation in NSWE models closely approximates the physical dissipation in breaking waves [50]. 
Simulations of tsunami propagation and coastal impact are performed in nested grids (Figs. 1. 3, by one-way coupling. In this method, time series of surface elevations and depth-averaged currents are computed at a large number of stations/numerical wave gages, defined in a coarser grid, along the boundary of the finer grid used in the next level of nesting. Computations are performed in the coarser grid and then are restarted in the finer grid, using the station time series as boundary conditions. As the latter include both incident and reflected waves computed in the coarser grid, this method closely approximates open boundary conditions. It was found that a nesting ratio with a factor 3-4 reduction in mesh size allowed achieving good accuracy in tsunami simulations. Finally, to prevent reflection in the first grid level, sponge layers are used along all the offshore boundaries (see, e.g., [52, 45, 46, 47] for a few examples).

For each coseismic source, simulations are initiated in a 1 arc-min (about $1800 \mathrm{~m}$ ) resolution grid, in which the tsunami source elevation is specified as an initial condition, with a zero velocity as is standard in such simulations). For the ACZ/LSB sources, this is the ocean basin scale spherical grid "Large G0", which covers the footprint of Fig. 1. and for the PRT/Caribbean arc sources, this is the smaller "Local G0" (Figs. 1. Table 4). In both grids, $200 \mathrm{~km}$ wide sponge layers are specified along the west, south and north offshore boundaries, and $400 \mathrm{~km}$ wide on the east boundary, to eliminate reflection. To compute the tsunami impact along the USEC, simulations are continued in 3 regional, angled, Cartesian nested grids of $450 \mathrm{~m}$ resolution, G1, G2, and G3 (Figs. 1, 3, Table 4).

For each grid level, bathymetry and topography are interpolated from the most accurate data available, of accuracy commensurate with the grid resolution. In deeper water, we use NOAA's 1 arc-min ETOPO-1 data (Fig. 1); in shallower water and on continental shelves, we use NOAA's NGDC 3" (about 90 m) Coastal Relief Model data (Fig. 3). Save points are defined in each grid (P-G1, P-G2, and P-G3 in Table 3.1. Fig. 1), where time series of surface elevations and currents are computed in order to verify that results in nested grids (G1, G2, G3) are consistent with those in the coarser parent grid (G0). Additional numerical wave gauges (P1 to P6 in Table 3.1, Fig. 1) are also specified to visualize details of incident tsunami wave trains (i.e., number of waves, wave amplitudes and periods), with P1 to P5 being located along the $200 \mathrm{~m}$ isobath, at the continental shelf edge, and P6 is in 1,000 m depth (southernmost point north of the Great Bahama Banks off of Florida).

Note that transformations from (Lon.-Lat.) to Cartesian $(x, y)$ coordinates and back are performed using Matlab's mapping toolbox, using a transverse Mercator projection (UTM), with an origin at each grid's centroid. 

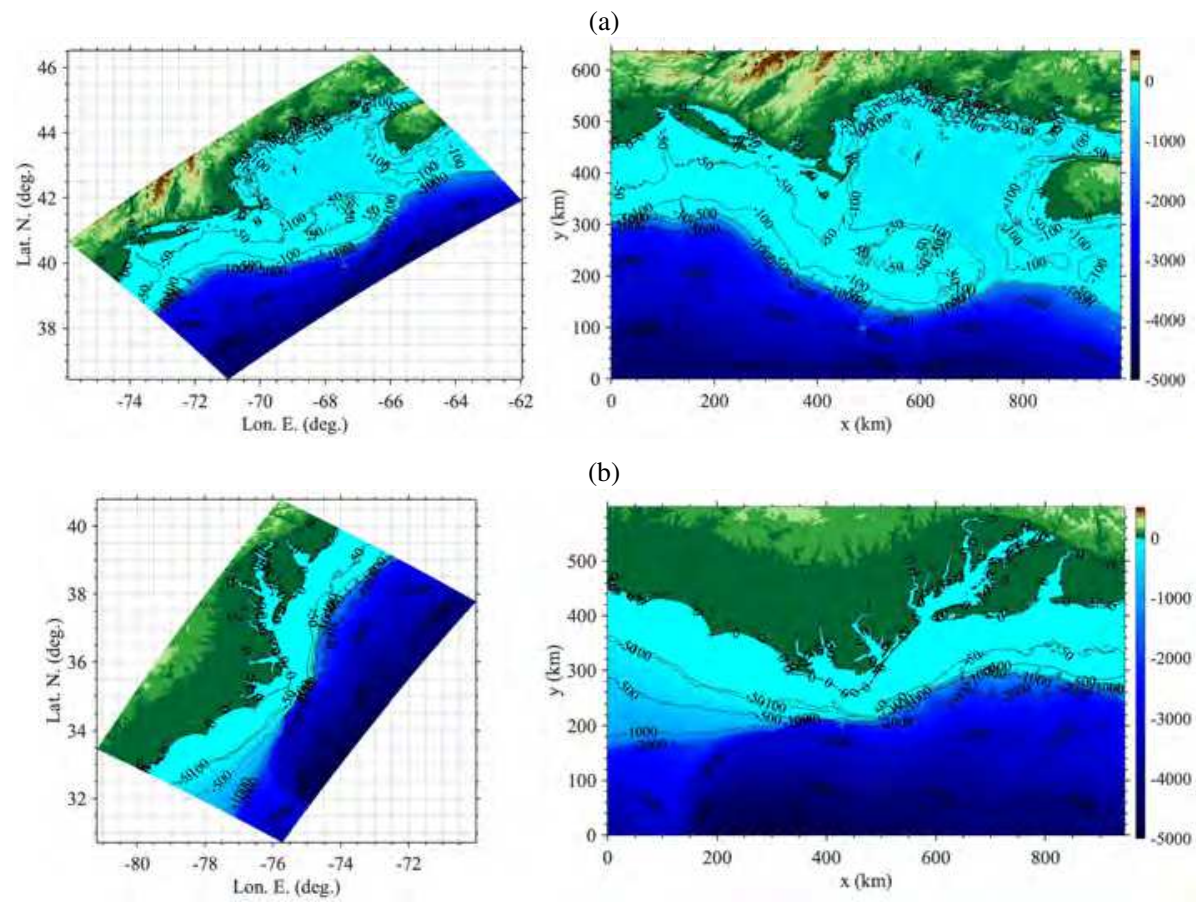

(b)
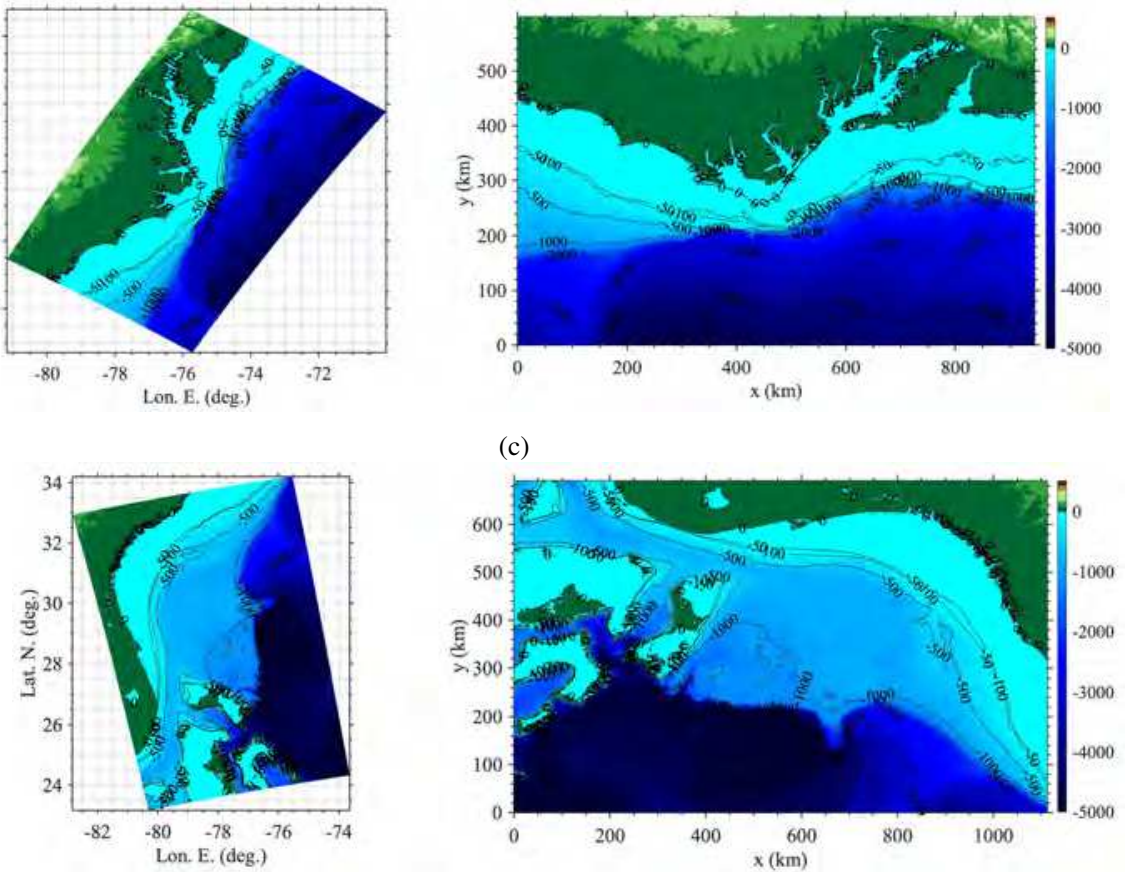

Fig. 3 Footprints of $450 \mathrm{~m}$ resolution grids used in FUNWAVE, in spherical (left) and Cartesian (right) coordinates (Table 4 Fig. 11: (a) G1, (b) G2, (c) G3; color scales and contours are bathymetry/topography in meter.

\subsection{Coseismic tsunami source parameters and generation}

Following the standard approach for large coseismic tsunamis, the initial tsunami surface elevation (with zero velocity) of each source is assumed to be equal to the maximum seafloor deformation caused by the earthquake; this is acceptable since water is nearly incompressible and raise times are usually small. The seafloor deformation is computed using Okada's [43] method, which solves linear three-dimensional (3D) elasticity problems in a series of 
(a)

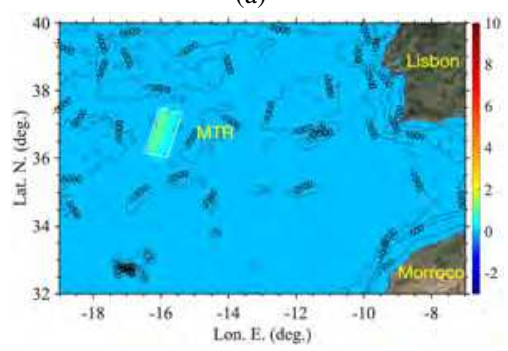

(c)

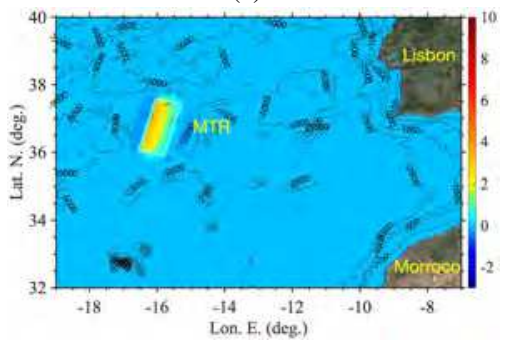

(e)

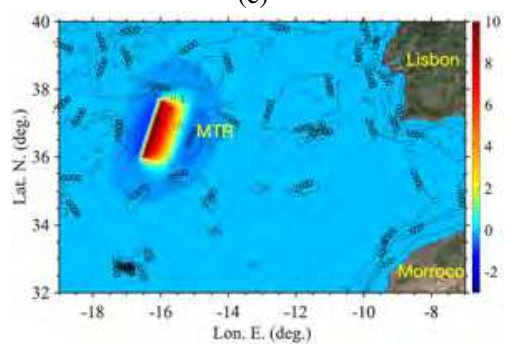

(g)

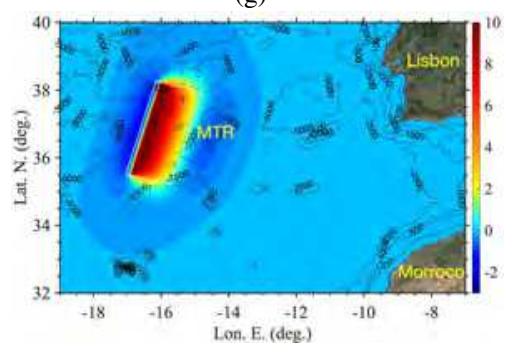

(i)

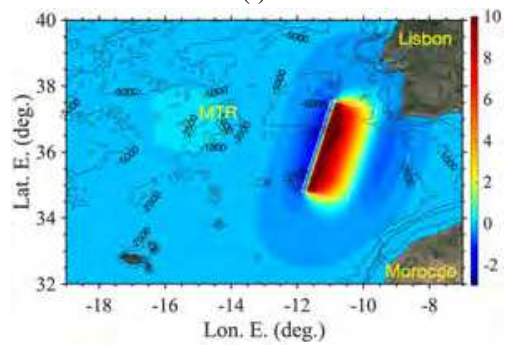

(b)

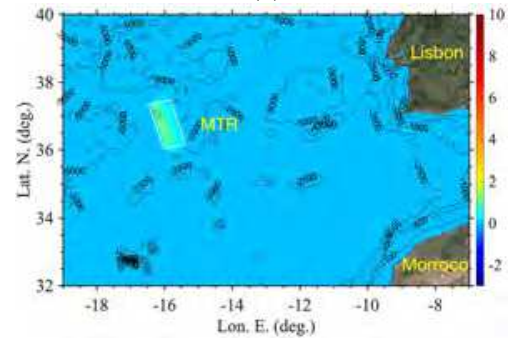

(d)

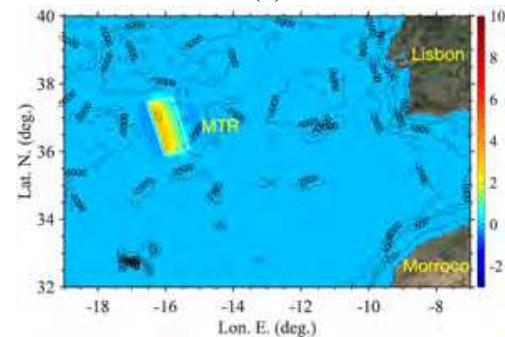

(f)

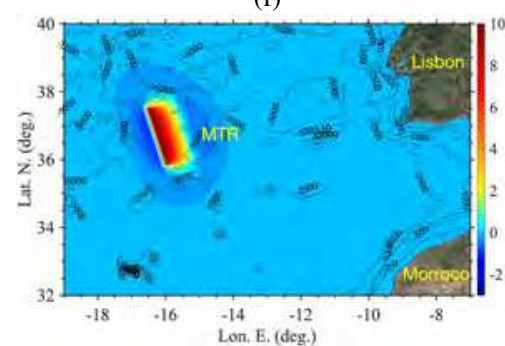

(h)

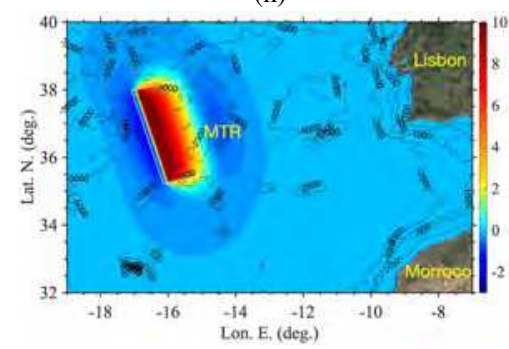

(j)

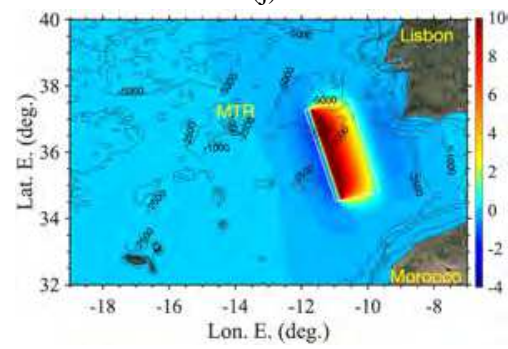

Fig. 4 Initial surface elevations computed with Okada's 43] method (color scale in meter) for coseismic tsunami sources in the ACZ/LSB area (Fig.1. Table11 : (a) M8.0-MTR1; (b) M8.0-MTR2; (c) M8.3-MTR1; (d) M8.3-MTR2; (e) M8.7-MTR1; (f) M8.7-MTR2; (g) M9.0-MTR1; (h) M9.0-MTR2; (i) M9.0-HSP1; (j) M9.0-HSP2. Each source has a single fault plane with footprint marked by white solid lines. Note, the M9.0 sources represent Lisbon 1755 proxies, located in either the ACZ, west of the MTR ("MTR"), or Horseshoe Plain ("HSP") areas (Fig. 1]) 
(a)

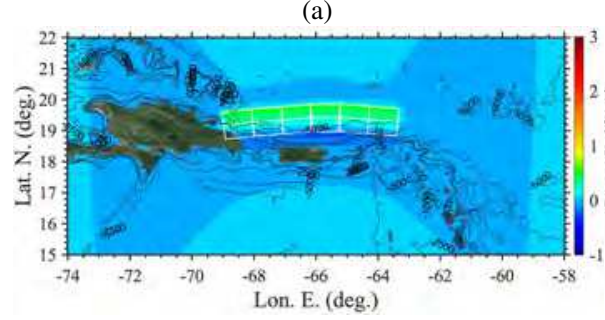

(c)

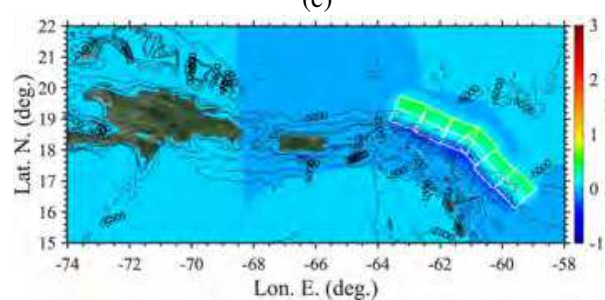

(e)

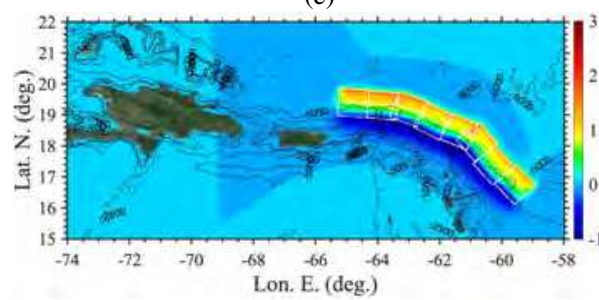

(g)

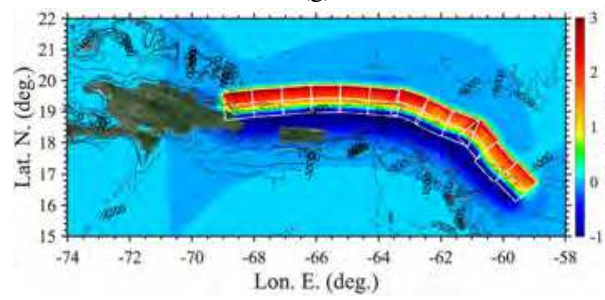

(b)

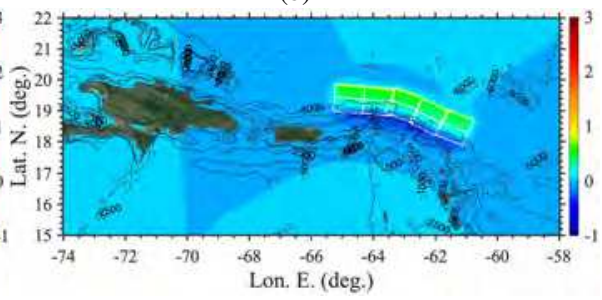

(d)

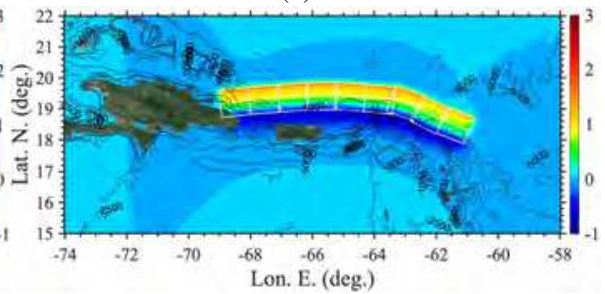

(f)

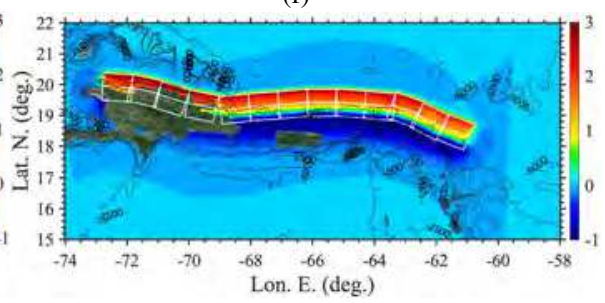

(h)

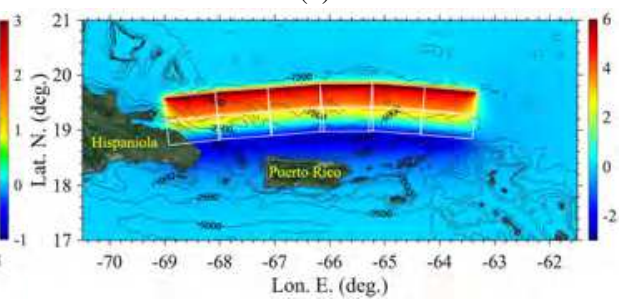

Fig. 5 Initial surface elevations computed with Okada's [43 method for the modeled PRT/Caribbean arc tsunami sources (Fig. 1. Table 3): (a) M8.3-PRT1; (b) M8.3-PRT2; (c) M8.3-PRT3; (d) M8.7-PRT1; (e) M8.7-PRT2; (f) M9.0-PRT1; (g) M9.0-PRT2; and (h) M9.0-PRT3. All source parameters are given Tables 2 and 3. Color scale (in meter), with same scale is used in plots (b-h), for comparison.

homogeneous half-spaces, corresponding to the source subfaults, each with a dislocation specified over an oblique plane. The problems are expressed as a set of Boundary Integral Equations, which are solved over a specific Cartesian grid encompassing the subfaults (here with a $1 \mathrm{~km}$ resolution); due to the linearity of the equations, the total seafloor deformation is expressed as the sum of that caused by each subfault. The required geometric parameters of each subfault are the fault plane horizontal area (width $W$ and length $L$ ), the depth $d_{o}$ at 
the source centroid, the centroid location $\left(x_{o}, y_{o}\right)$ (Lon.-Lat.), and 3 angles orientating the fault plane (dip $\delta$, rake $\gamma$, strike $\theta$ ); with these definitions, $d_{o}=d+(W / 2) \sin \delta$, where $d$ is the highest point on the subfault plane. In addition the model needs the Coulomb modulus $(\mu)$ of the medium (10-60 Gpa, for shallow ruptures in soft/poorly consolidated marine sediment to deep ruptures in basalt); $\mu=40 \mathrm{GPa}$ is used here in all cases [17].

Parameters of selected coseismic sources modeled in the ACZ/LSB and the PRT/Caribbean arc areas are given in Table 1 , and 2 , 3, respectively. Fig. 4 shows the initial surface elevations computed for the ACZ/LSB sources (Table 1). The four largest M9 sources cause a nearly $12 \mathrm{~m}$ maximum elevation and a $4 \mathrm{~m}$ maximum depression. The other, smaller magnitude, ASZ sources plotted in Figs. 4 4 -j have similar patterns of uplift and subsidence, but smaller surface elevations distributed over gradually smaller areas. Fig. 5 shows the initial surface elevations computed for the PRT/Caribbean arc sources (Tables Tables 2 and 3). The new M9 sources in Figs. 5F,g (PRT1 and PRT2) have an over $4 \mathrm{~m}$ maximum elevation and $2 \mathrm{~m}$ maximum depression, about half the maximum value of the earlier M9.0-PRT3 PMT source (Fig.5h). The other, smaller magnitude, sources plotted in Figs. 5h-e have similar patterns of uplift and subsidence, but gradually smaller surface elevations as their magnitude decreases.

\subsection{Tsunami propagation and coastal impact}

For each coseismic source, tsunami propagation is first simulated with FUNWAVE in grids Large G0 or Local G0 for $24 \mathrm{~h}$, respectively, depending on the source location. Simulations are then restarted in the three finer nested grids (G1, G2 and G3), also up to the full duration, but for more efficiency starting from the time of first arrival of the tsunami along each nested grid boundary.

Figure 6 shows an example of surface elevations computed for the Lisbon 1755 proxy source M9-HSP1 (Fig. 4; Table 1 ) at times $t=1$ to $11 \mathrm{~h}$, with the figures at later times focusing on the impact computed in grid G3 off of Florida. The snapshots illustrate how waves radiate away from the source, diffracting around islands and refracting as a function of the bathymetry. In particular, Figs. 6e-h illustrate how the continental slope and shelf cause intense refraction of incident tsunami waves, which increasingly bend to become nearly parallel to the isobaths as they gradually slow down. This phenomenon affects all ACZ/LSB sources in the same manner and causes them to focus or defocus their energy on identical coastal areas depending on the shelf bathymetry. As a result of dispersion, nearshore tsunami waves are made of at least three major crests and troughs (Fig. 64). Despite the $15 \mathrm{deg}$. strike angle, the largest tsunami waves originated from this source are aimed at Florida. This is confirmed in Fig. 7] which shows the envelopes of maximum surface elevations computed for the M9-HSP1 and M9-HSP2 sources. While both sources direct their major 

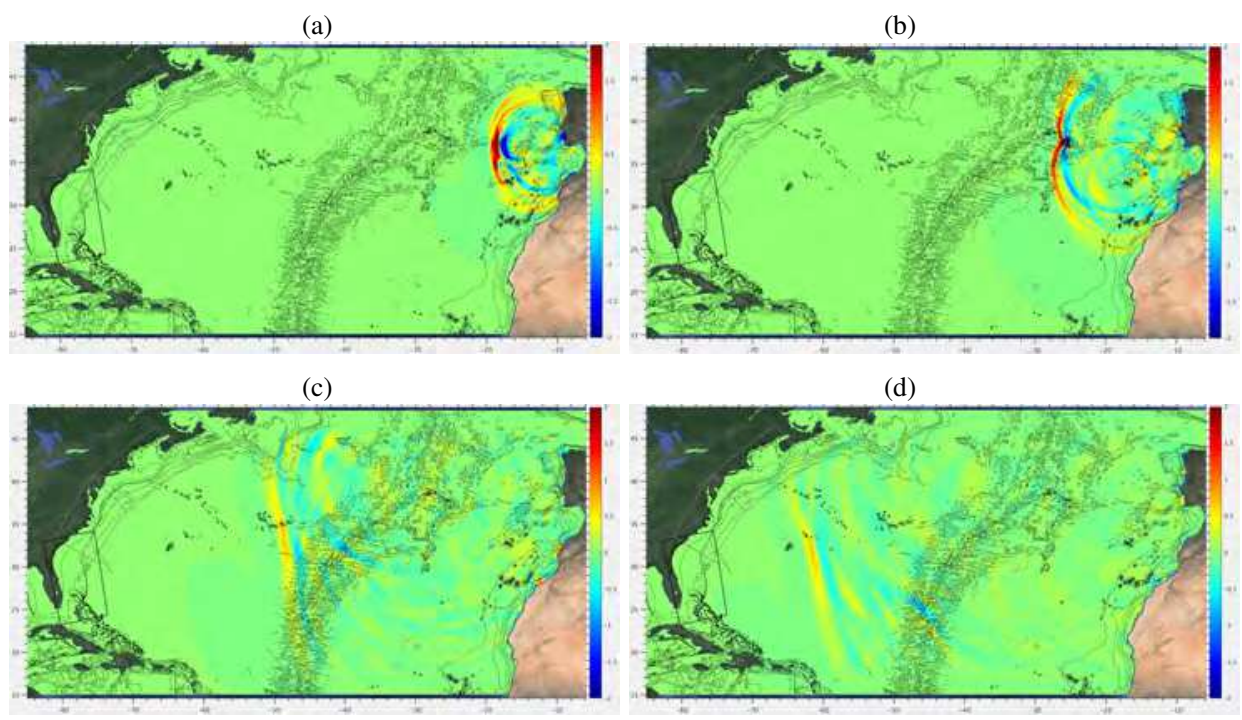

(e)

(f)
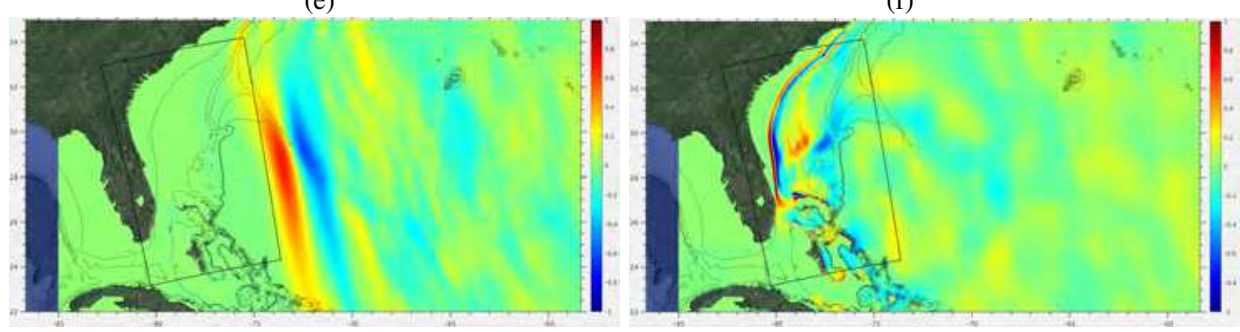

(g)

(h)
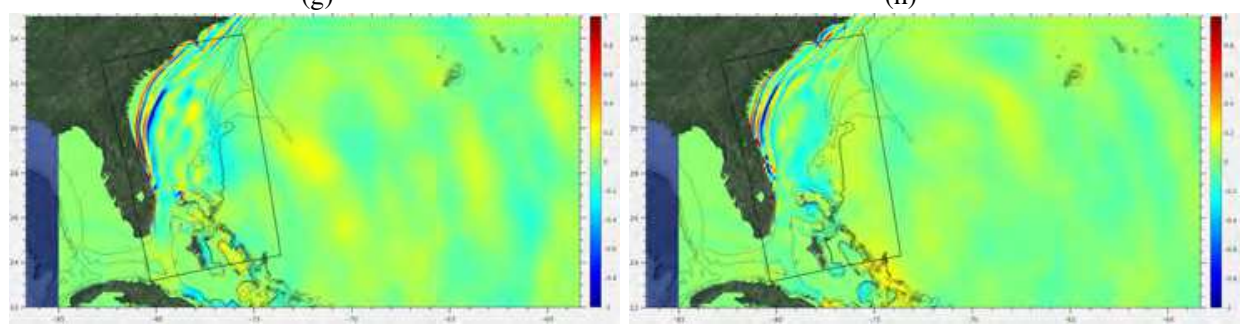

Fig. 6 Snapshots of surface elevations (color scale in meter) computed for the Lisbon 1755 proxy source M9-HSP1 (Fig. 4; Table 1), at $t=$ (a) 1, (b) 2, (c) 5, (d) 6.5, (e) 8, (f) 9.5, (g) 10.5, and (h) 11 h. Some isobaths are plotted for reference, but without labels to simplify the figures. Higher resolutions results are used wherever available.

wave energy southwestward, as expected from their orientation, the first source, with a 15 deg. strike angle, causes a relatively larger tsunami impact along the upper USEC than the second source, with a $345 \mathrm{deg}$. strike angle, which causes a larger impact along the lower USEC and the Caribbean Islands. This will be further detailed later. A movies showing 
(a)

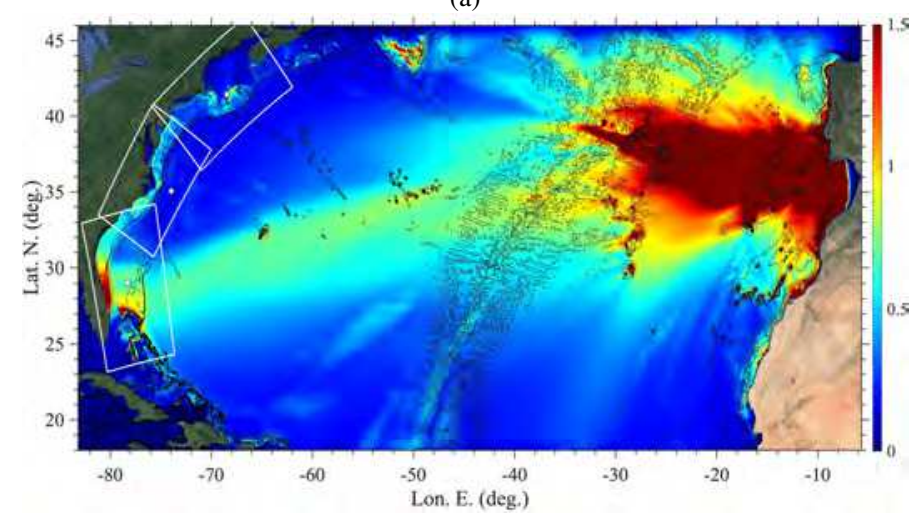

(b)

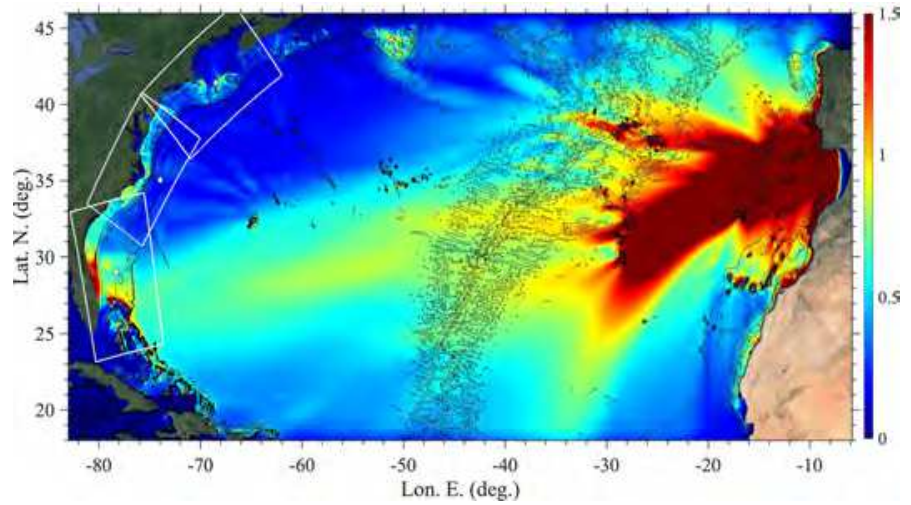

Fig. 7 Envelope of maximum surface elevation computed with FUNWAVE during 24h of simulations in grid Large G0 (Table 4 Fig. 1 for the: (a) M9-HSP1, and (b) M9-HSP2, historical Lisbon 1755 coseismic sources (with parameters listed in Table 1 and initial surface elevations in Figs.4,, j). Color scales are surface elevation in meter.

animated results of the M9-HSP1 source simulation is provided as supplementary material (HSP1.mp4).

Similarly, Fig. 8 shows snapshots of surface elevations for the M9.0-PRT3 source (Fig. 5h; Table 3), at $t=0.5$ to $5 \mathrm{~h}$, with the figures at later times focusing on grid G1, off of the upper USEC (centered in New England) where, due to the northward directionality of tsunami energy for this source, the most impacted areas are located. This is further confirmed in the maximum envelope of surface elevation shown in Fig. 9 for the similar M9-PRT2 source. A movies showing animated results of the M9-PRT3 source simulation is provided as supplementary material (PRT3.mp4).

As for the ACZ/LSB sources, Figs. 85-e show that an intense refraction of incident tsunami waves occurs at the shelf break and over the continental shelf, which makes wave crests increasingly parallel to the local isobaths. Comparing these results to Figs. 6-h, we 


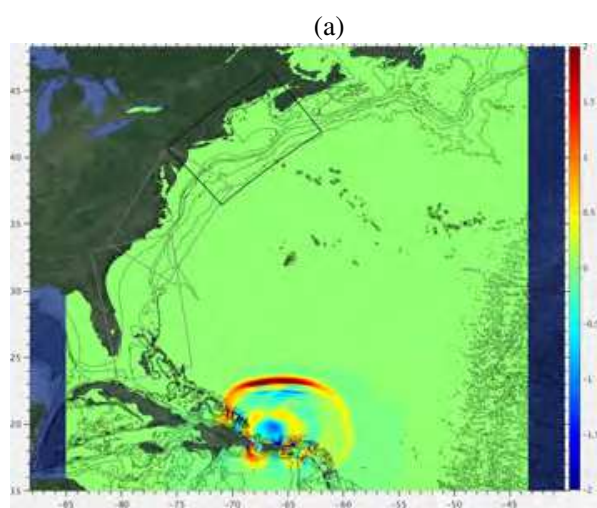

(c)

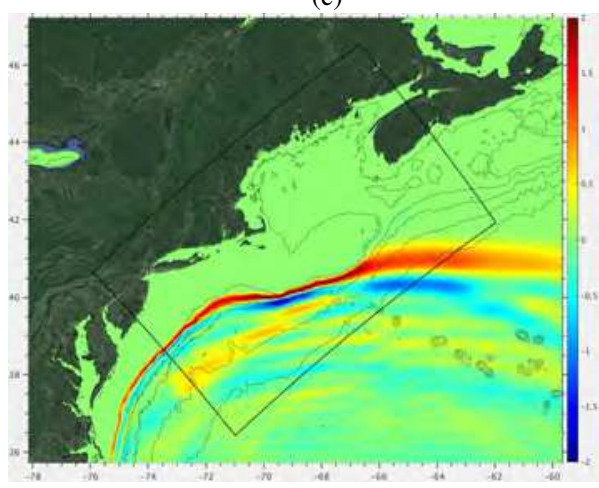

(e)

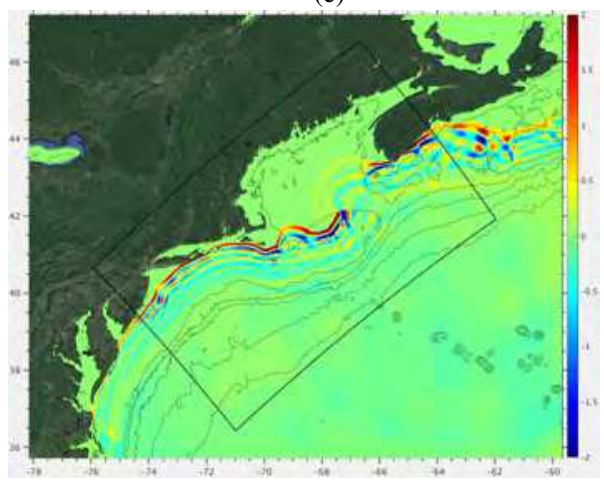

(b)

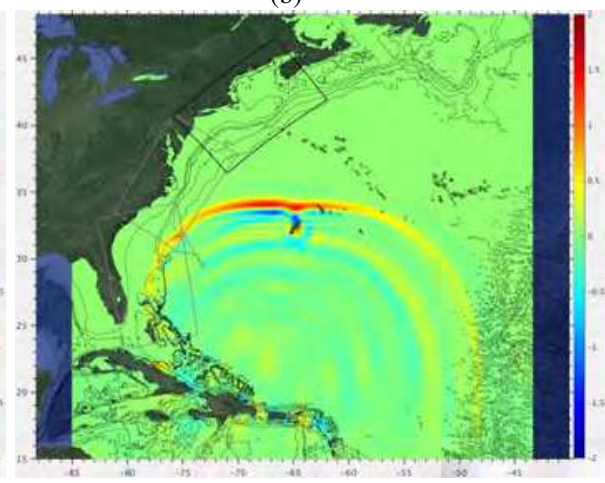

(d)

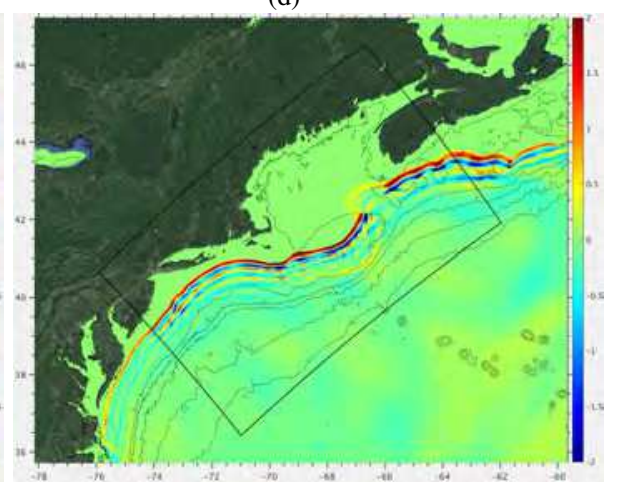

(f)

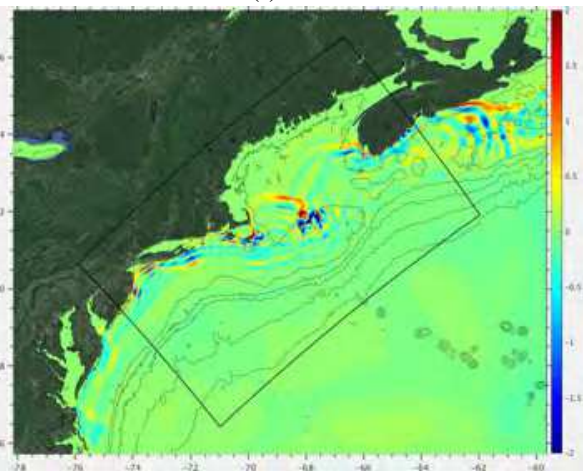

Fig. 8 Snapshots of surface elevations (color scale in meter) for the PRT/Caribbean arc source M9-PRT3 (Table 3; Fig. 5h), at $t=$ (a) 0.5 , (b) 2, (c) 3, (d) 4, (e) 4.5, and (f) $5 \mathrm{~h}$. Some isobaths are plotted for reference, but without labels to simplify the figures. Higher resolutions results are used wherever available. 


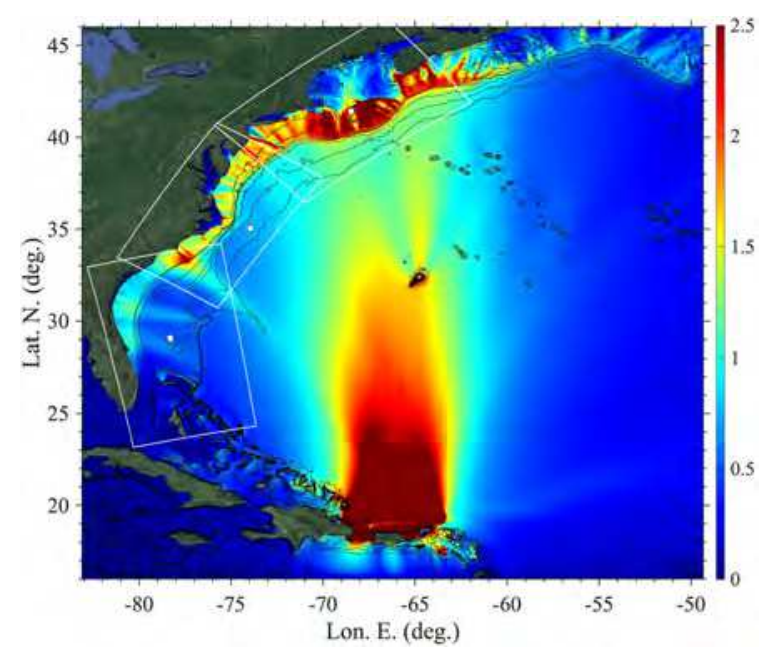

Fig. 9 Envelope of maximum surface elevation computed with FUNWAVE during 24h of simulations in grid Local G0 (Table 4 Fig. 1 for the M9-PRT3 coseismic source (with parameters listed in Table 3 and initial surface elevations in Figs. 5h). Color scales are surface elevation in meter.

see that wave focusing-defocusing caused by refraction over the wide and shallow shelf, particularly around ridges and canyons in the bottom bathymetry, yields a corresponding modulation of tsunami coastal impact that is independent from the initial tsunami directionality. Detailed results of coastal impact, discussed later, will confirm that these modulation patterns are identical for the ACZ/LSB or PRT sources, which are originated eastward and southward, respectively. This leads to the same areas of the coast always being subjected to more or less tsunami hazard, whatever the tsunami source origin. This phenomenon was already noted in earlier work, for other tsunami sources affecting the USEC [27,54,45].

Finally, for each source, the relevance of the one-way coupling method is verified by comparing time series of surface elevations at the 9 save points (Table 3.1), in the coarser grids Larger G0/Local G0 and the finer nested grids G1, G2 and G3. For instance, Figures 10 and 11 show time series of surface elevations computed for the M9-HSP1 and M9.0PRT1 sources. At most locations, there is a good agreement of the coarse and finer grid simulations for the first few hours of tsunami impact. Once reflected waves from the coast propagate back to the save points, however, differences become larger as reflection is more accurately computed in the finer grids. Differences are largest at point P-G1, which is on the shelf in shallower water and closer to shore, east of Cape Cod. Overall, the agreement of time series computed in different grids confirms the relevance of the one-way coupling methodology in nested grids used here. Time series of surface elevations at save points look qualitatively similar for the other ACZ/LSB and PRT sources and will not be repeated here. 

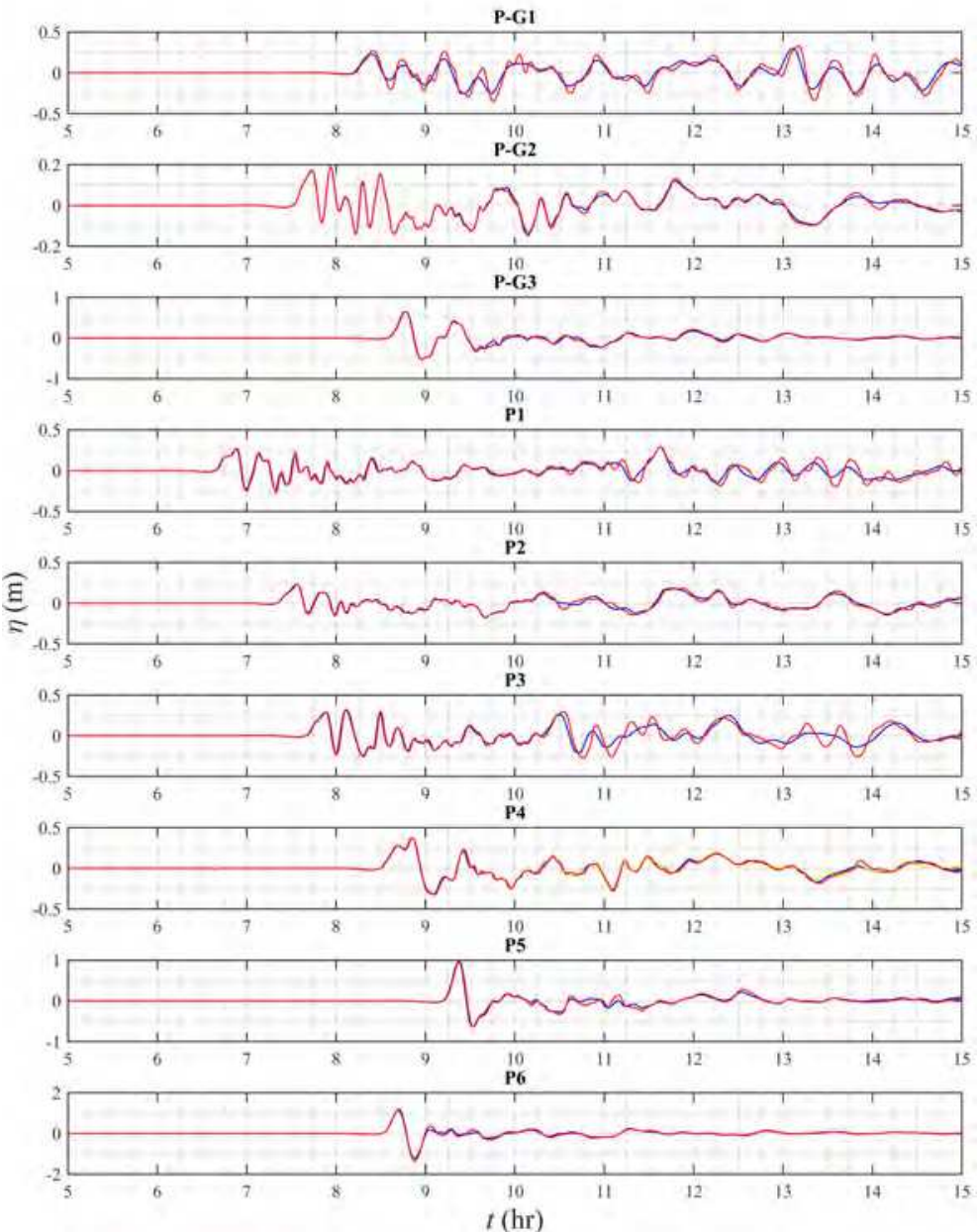

Fig. 10 Time series of surface elevations computed for the M9-HSP1 source (Table 1), at the 9 save points defined in Table 5 (shown in Figs. 11: (blue lines) in grid Large G0; (red lines) in nested grids G1-G3. For point P4, yellow indicates surface elevation computed in G2, red indicates surface elevation computed in G3.

\section{Results of tsunami coastal impact}

4.1 Tsunami hazard metrics

\subsubsection{Definition of hazard metrics and classes}

For each of the ten ACZ/LSB (Table 11, Fig. 4 and eight PRT/Caribbean arc coseismic sources (Table 3; Fig. 5p simulated with FUNWAVE, coastal impact was computed in the three nested grids (G1, G2, G3) along the $5 \mathrm{~m}$ isobath that parallels the USEC (considering the coarse coastal grids used here). FUNWAVE results were interpolated at a large number 

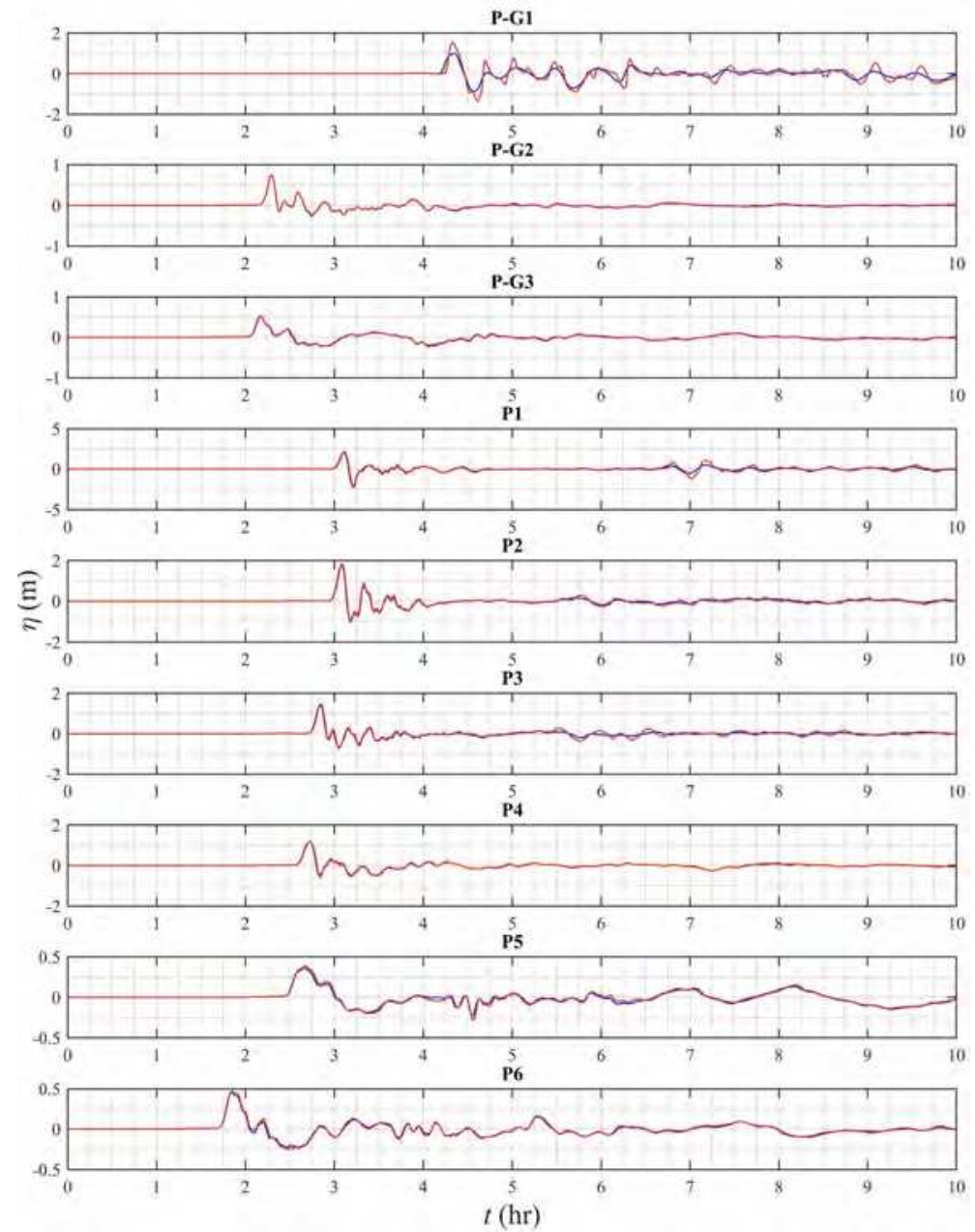

Fig. 11 Time series of surface elevations computed for the M9-PRT3 source (Table 3), at the 9 save points defined in Table 5 (shown in Figs. 1): (blue lines) in grid Local G0; (red lines) in nested grids G1-G3. For point P4, black indicates surface elevation computed in G2, red indicates surface elevation computed in G3.

of coastal save points defined by their latitude-longitude coordinates along the isobath. For clarity, in most of the results and figures, $J=18,201$ save points are used, which exclude large bays (i.e., Cheasapeake and Delaware bays, and Long Island Sound); when large bays are considered, an additional 10,210 save points are specified within the bays $(J=28,411)$. Based on these results, values of four tsunami hazard metrics $M_{i}(i=1, \ldots, 4)$ were computed for each source along the isobath: (1) maximum tsunami elevation $M_{1}=\eta_{\max }$, (2) current $M_{2}=U_{\max }$, and (3) momentum force $M_{3}=F_{\max }$, as well as (4) $M_{4}=1 / t_{a}$, function of arrival time $t_{a}$ (here a large arrival time corresponds to a low hazard). These metrics quantify inundation hazard, navigational hazard nearshore and in harbors, hazard 
(a)

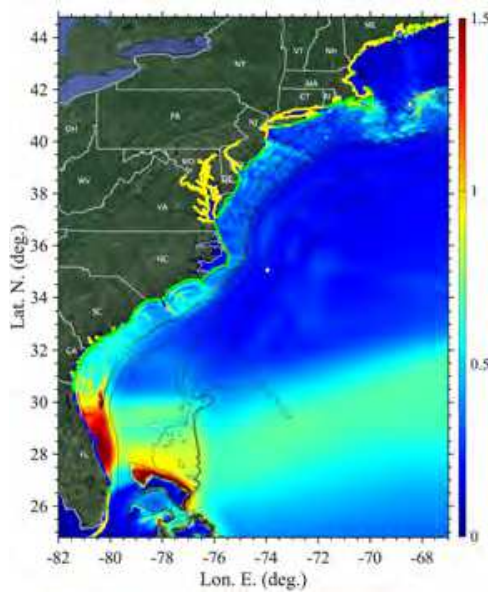

(b)

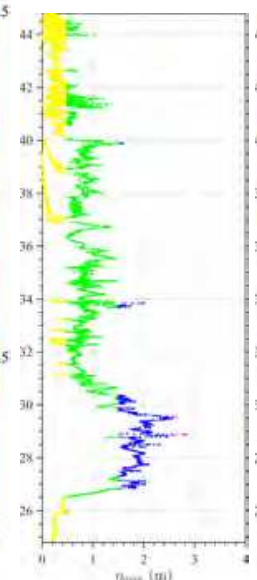

(c)

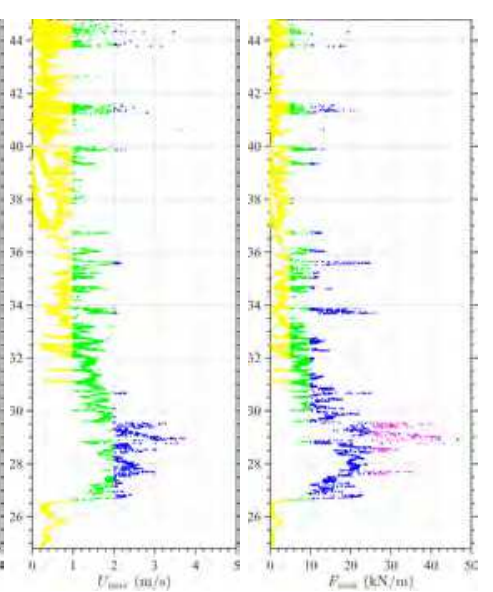

Fig. 12 Hazard metrics along the USEC for the M9.0-HSP1 coseismic source (Table 1 Fig. 4) : (a) envelope of maximum surface elevations $\eta_{\max }$ over grids G1,G2,G3 (color scale in meter; colored dots along the coast correspond to hazard intensity $C_{k}^{1}$ from (b)); (b,c,d) color coded hazard intensity classes $C_{k}^{i}(k=1, \ldots, 5$; yellow, green, blue, magenta and red; Table 6 with corresponding values of: $(i=1) \eta_{\max },(i=2) U_{\max }$, and $(i=3) F_{\max }$, respectively, in the same grids; $J=28,401$ save points are defined along the $5 \mathrm{~m}$ isobath, here including large bays.

(a)

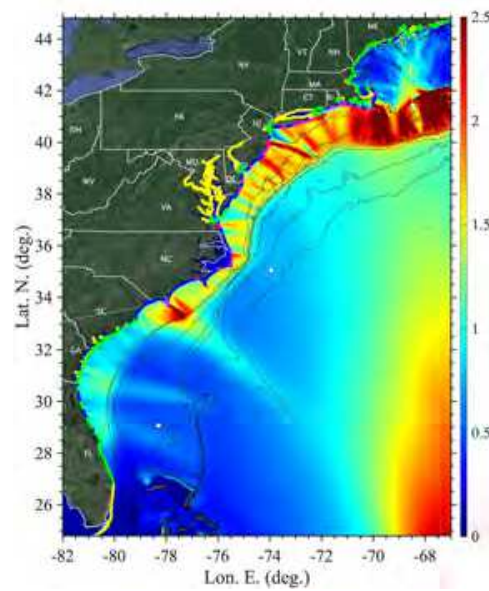

(b)

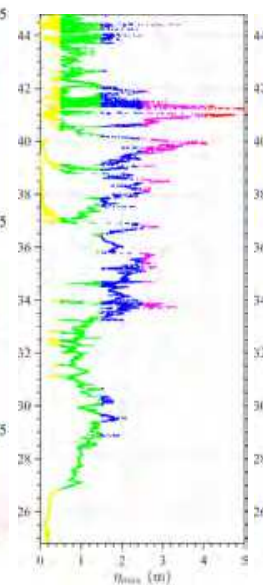

(c)

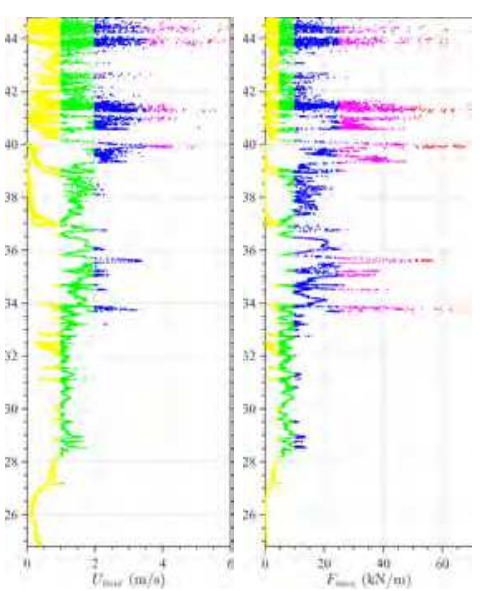

Fig. 13 Same results as in Fig. 12 for the M9.0-PRT3 coseismic source (Table 3; Fig. 54).

from tsunami impact forces on structures, and hazard resulting from low warning time, respectively.

FUNWAVE outputs directly provide $\eta$ and horizontal current components $(u, v)$ (at 0.531 times the local depth) at each grid point as a function of time, based on which $\eta_{\max }$, $U_{\max }=\max \left\{\sqrt{u^{2}+v^{2}}\right\}$, and $F_{\max }=\max \left\{\rho(d+\eta) U^{2}\right\}$ are computed over the en- 


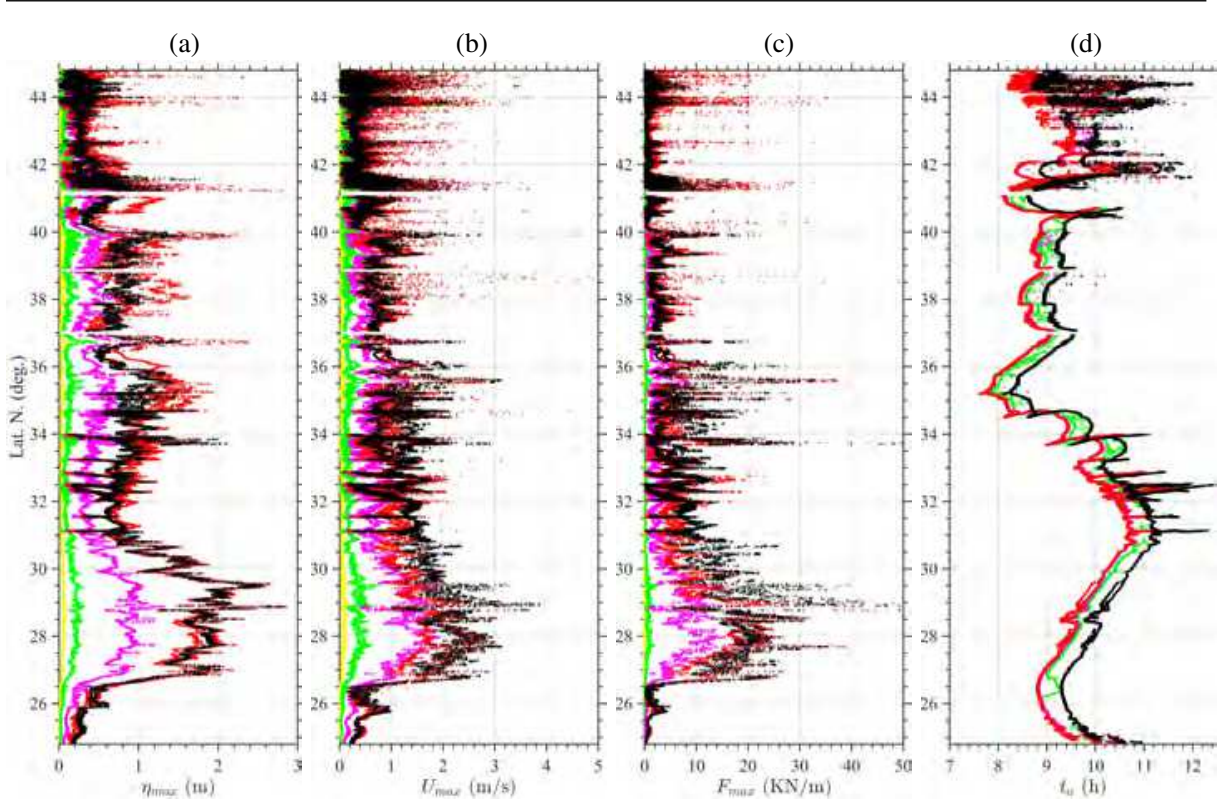

Fig. 14 Envelope at the $5 \mathrm{~m}$ isobath ( $J=18,201$ save points excluding large bays) of maximum tsunami: (a) elevation; (b) current; (c) momentum force; and (d) arrival time, computed in grids G1,G2,G3, for the ten ACZ/LSB sources (Table 1] Fig. 4): (green) M8.0-MTR1, M8.0-MTR2; (yellow) M8.3-MTR1, M8.3MTR2; (magenta) M8.7-MTR1, M8.7-MTR2; (red) M9.0-MTR1, M9.0-MTR2; and (black) M9.0-HSP1, M9.0-HSP2. Note, arrival times for the latter two sources, which are slightly longer, are not shown for clarity.

tire duration of simulations at all grid point. Figures 7 and 9 , show examples of maximum surface elevations computed over the entire computational domain for some of the tsunami sources. These results are then interpolated at the save points along the $5 \mathrm{~m}$ isobath. Tsunami arrival time is calculated along the same isobath, as the time when a positive or negative surface elevation first occurs over a threshold, i.e., $t_{a}$ (in hours) is the minimum time such that $\left|\eta\left(t_{a}, x_{j}, y_{j}\right)\right| \geq \Delta \eta$; where $\left(x_{j}, y_{j}\right)(j=1, \ldots, J)$ denotes the save points along the isobath and here $\Delta \eta=0.01 \mathrm{~m}$. Since tsunamis are very small amplitude waves relative to depth in most of their propagation, their celerity is well approximated as a function of depth by the linear long wave celerity, $c=\sqrt{g d}$, which is not amplitude dependent; hence different tsunamis originated from the same area propagate similarly along the same "wave rays". This similarity of propagation to shore is further reinforced by refraction that takes place in large depth for long tsunami waves and causes each tsunami to propagate similarly over the wide USEC shelf, whatever its origin. Consequently, tsunamis of different magnitude originated from the same area, LSB/ACZ or PRT/Caribbean arc, should have very similar arrival times along the USEC, which will be verified in results. One caveat is, for the weakest LSB/ACZ M8 and M8.3 sources that approach areas of the USEC featuring bays and more complex shoreline geometries, with a small amplitude, and hence only reach the 


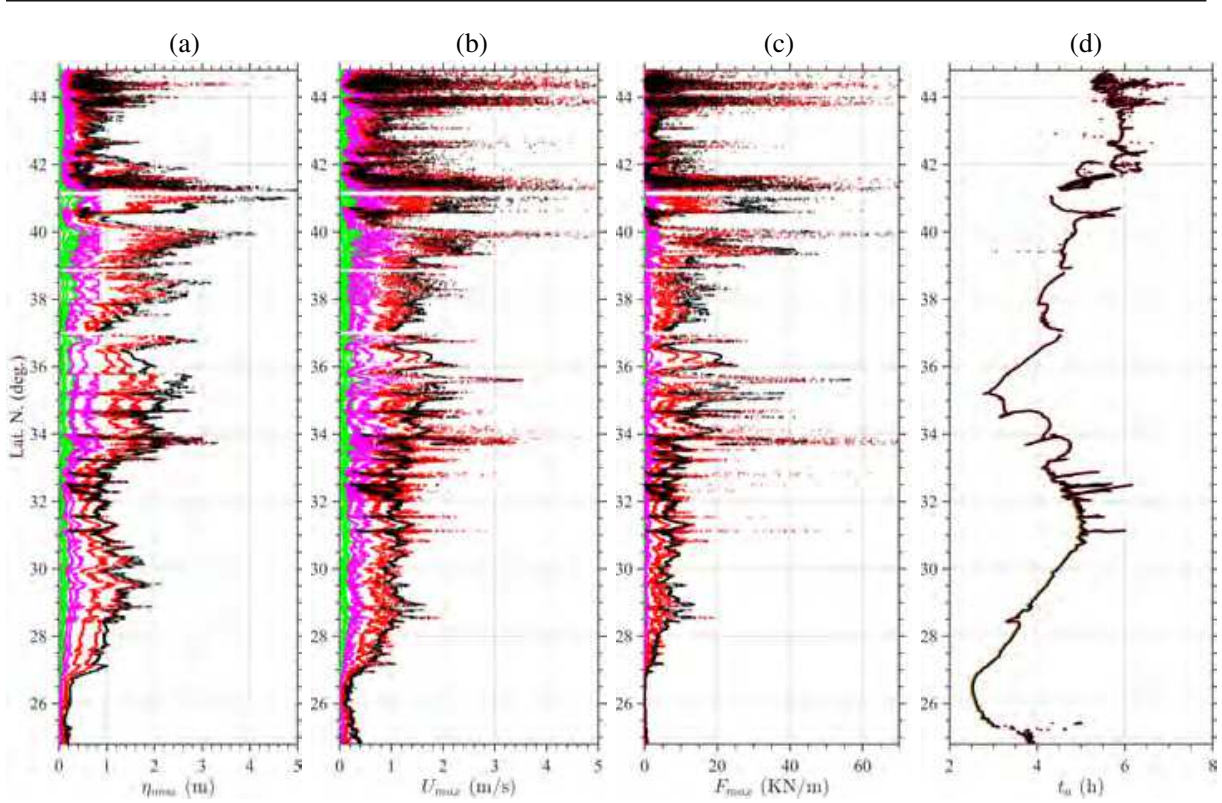

Fig. 15 Envelope at the $5 \mathrm{~m}$ isobath ( $J=18,201$ save points excluding large bays) of maximum tsunami: (a) elevation; (b) current; (c) momentum force; and (d) arrival time, computed in grids G1,G2,G3, for the eight PRT/Caribbean arc sources (Table 2.2. Fig. 5): (green) M8.3-PRT1, M8.3-PRT2, M8.3-PRT3; (magenta) M8.7-PRT1, M8.7-PRT2; (red) M9.0-PRT2, M9.0-PRT2; and (black) M9.0-PRT3. Note, arrival times for the latter source, which is similar, is not shown for clarity.

\begin{tabular}{|c||c|c|c|c|c|}
\hline Hazard Metric & $C_{1}^{i}$ & $C_{2}^{i}$ & $C_{3}^{i}$ & $C_{4}^{i}$ & $C_{5}^{i}$ \\
\hline \hline$M_{1}=\eta_{\max }(\mathrm{m})$ & {$[0-0.5[$} & {$[0.5-1.5[$} & {$[1.5-2.5[$} & {$[2.5-4[$} & $>4$ \\
$M_{2}=U_{\max }(\mathrm{m} / \mathrm{s})$ & {$[0-1[$} & {$[1-2[$} & {$[2-3.5[$} & {$[3.5-5[$} & $>5$ \\
$M_{3}=F_{\max }(\mathrm{kN} / \mathrm{m})$ & {$[0-5[$} & {$[5-10[$} & {$[10-25[$} & {$[25-50[$} & $>50$ \\
$M_{4}=1 / t_{a}(1 / \mathrm{h})$ & {$[0-0.1[$} & {$[0.1-0.17[$} & {$[0.17-0.25[$} & {$[0.25-0.50[$} & $>0.50$ \\
\hline
\end{tabular}

Table 6 Class limits $\left[C_{k, \text { min }}^{i}-C_{k, \text { max }}^{i}[\right.$, with $(k=1, \ldots, 5)$ of hazard intensity (low, low-medium, medium, high, to highest hazard), for four hazard metrics $M_{i}(i=1, \ldots, 4)$ used to compute the Tsunami Intensity Index (TII) with Eq. 3.

$\Delta \eta$ threshold later in their propagation, due to shoaling and reflection of the tsunami wave trains. This tends to increase the arrival time for these sources in some areas. Details will be shown later.

Once the four hazard metrics computed, to more easily identify areas facing lesser or larger hazard, similar to classes defined by Boschetti et al. [10] for the first two metrics in their tsunami intensity scale, we define five intensity classes, referred to as $C_{k}^{i} ; k=1, \ldots, 5$ for each of the four metrics, with ranges of values $\left[C_{k, \min }^{i}-C_{k, \max }^{i}\right.$ [ from low to increasing hazard severity (Table 6). These classes of hazard for each metric can also be referred to as: 
low, low-medium, medium, high, and highest hazard. As discussed in Boschetti et al. [10], who reviewed other relevant work to date, the selected values for maximum inundation

$\eta_{\max }$ correspond, for adult pedestrians, to being up to knee-tight deep for class $C_{1}^{1}$, up to chest/head deep for class $C_{2}^{1}$, up to head to overhead deep for $C_{3}^{1}$ while loosing the ability to feel the terrain, and then very deep; in classes $C_{3}^{1}$ and higher, people would be forced to swim or have to find high ground or vertically evacuate to be safe. Likewise, for maximum currents $U_{\max }$ (see also Lynett et al. [38]), adult pedestrians would only be able to fight the current in classes $C_{1}^{2}$ and $C_{2}^{2}$, while navigation would start being gradually and then severely impended nearshore and in harbors for classes $C_{2}^{2}$ and above. Maximum momentum force $F_{\max }$ classes correspond to most structures resisting up to most structures suffering significant damage or destruction, except for the strongest concrete or steel-built (also elevated) structures. Finally, regarding arrival time, the classes reflect going from large enough time $(10 \mathrm{~h})$ to evacuate most of the population from high hazard areas to not having enough time $(2 \mathrm{~h})$ for warning and evacuating a meaningful fraction of the population at risk. For each source considered here, and for their envelopes, values of the four metrics $M_{i}$ were computed along the $5 \mathrm{~m}$ isobath and then sorted out by class. Plots of each metric along the isobath were made, which were color coded as a function of the corresponding class for the metric, $C_{k}^{i}$ : yellow, green, blue, magenta, and red, for $k=1,2,3,4,5$.

\subsubsection{Overall results of hazard metrics and classes}

Figures 12 and 13 show examples of results of the first 3, physical, metrics computed computed in grids $\mathrm{G} 1, \mathrm{G} 2, \mathrm{G} 3$, at $J=28,401$ save points on the $5 \mathrm{~m}$ isobath, here in including large bays, for the M9-HSP1 ACZ/LSB source (Table 1] Fig. 4]) and the M9.0-PRT3 PRT/Caribbean arc source (Table 3; Fig. 5h). In each figure, panel (a) shows the envelope of maximum elevation, and panels (b,c,d) show the maximum elevation, current, and force, at the $5 \mathrm{~m}$ isobath, color-coded with their hazard class intensity $C_{k}^{i}$ value; the class intensity value of surface elevation along is also marked in (a) along the coast.

As for the envelope in grids Local/Large G0 shown in Figs. 7 and 9, we first observe an overall effect of the tsunami directionality on coastal impact, with the $15 \mathrm{deg}$. strike ACZ/LSB source (M9.0-HSP1) affecting more the lower USEC and the Caribbean Islands, and the PRT source (M9.0-PRT3) affecting more the upper USEC. Additionally, as before, for both sources, there is a fine scale modulation of tsunami impact along the USEC, as a function of the bathymetry (particularly on the shelf break and shelf) due to refraction causing wave focusing/defocusing on specific areas of the coast with convex/concave isobath geometry, respectively. For both of these sources and for the three plotted metrics, there are many locations in the 3rd and 4th hazard class and a few in the 5th, highest hazard class for 
maximum elevation and current. This is expected and these are among the largest magnitude sources. Computed results look qualitatively similar for the other, smaller magnitude, ACZ/LSB and PRT sources and will not be detailed here individually. However, their overall impact on the USEC is compared with each other next.

Thus, Figs. 14 and 15 show a comparison of tsunami coastal impact at $J=18,201$ save points along the $5 \mathrm{~m}$ isobath, here excluding large bays, for the four hazard metrics $M_{i}$, of the ten ACZ/LSB sources (Table 1) and eight PRT/Caribbean arc sources (Table 3), respectively. Tables 7 and 8 provide statistics computed based on these results, for the: mean, standard deviation, root-mean-square and average of the top 33, 10, 1 and 0.1 percentiles, of the first three metrics. For the 3 physical hazard metrics, results in Tables 7 and 8 show the expected trend of the statistics with the intensity of each metric statistics increasing with the source magnitude, except for 0.1 percentile results which only average 18 individual results and hence are more sensitive to a few noisy results. One exception is the two M9.0-HSP1 and -HSP2 sources in the ACZ area, which due to the effect of the MTR, cause a slightly lower overall impact on the USEC than the two M9.0-MTR1 and -MTR2 sources which are sited west of the MTR. Due to sources' directivity and refraction, the overall impact of ACZ/LSB sources in larger on the southern USEC (south of $35 \mathrm{deg}$. N), whereas it is the opposite for the PRT/Caribbean arc sources.

Regarding the arrival time metric, Figs. 14 1 show, as expected, that all the arrival times are quite close to each other, ranging between 7.75 and $12.5 \mathrm{~h}$, except for a few larger spurious values for the weakest ACZ sources. Note that overall, the M9 sources located further east in the HSP have an arrival time about 30 min larger than for the M9 sources located in the MTR. The arrival times for the ACZ/LZB sources are mostly in hazard class 2 , with a smaller fraction of values in hazard class 1 . For the PRT sources, Fig. 15d shows that arrival times are shorter than for ACZ sources (2.5-7.25 h), but follow a similar pattern along the coast, due to the similar refraction over the wide shelf. The PRT arrival times are nearly the same for all sources (within a few minutes form each other) and fall mostly within the medium and high hazard classes (3 and 4).

\subsubsection{Detailed results of hazard metrics and classes}

For ACZ sources, results of the 3 physical metrics in Figs. 14 a-c and in Table 7 show that the two M8.0 sources, which have 32 times less energy than the M9.0 sources (see Eq. 1), only cause $\mathrm{a} \sim 0.03 \mathrm{~m}$ average maximum elevation along the USEC, with a 0.03 or 0.013 $\mathrm{m}$ standard deviation, and the two M8.3 sources, which have 11 times less energy than the M9.0 sources, $\mathrm{a} \sim 0.09 \mathrm{~m}$ average maximum elevation with a 0.07 standard deviation. Average maximum currents and forces are commensurate with these values and small for 


\begin{tabular}{|c|c|c|c|c|c|c|c|}
\hline$\eta(\mathrm{m})$ & $\mu_{\eta}$ & $\sigma_{\eta}$ & $\eta_{r m s}$ & $\eta_{1 / 3}$ & $\eta_{1 / 10}$ & $\eta_{1 / 100}$ & $\eta_{1 / 1000}$ \\
\hline M8.0-MTR1 & 0.032 & 0.031 & 0.044 & 0.053 & 0.079 & 0.205 & 0.637 \\
\hline M8.0-MTR2 & 0.025 & 0.013 & 0.028 & 0.040 & 0.054 & 0.072 & 0.085 \\
\hline M8.3-MTR1 & 0.091 & 0.068 & 0.113 & 0.161 & 0.230 & 0.438 & 0.975 \\
\hline M8.3-MTR2 & 0.085 & 0.061 & 0.104 & 0.148 & 0.217 & 0.369 & 0.7234 \\
\hline M8.7-MTR1 & 0.319 & 0.218 & 0.386 & 0.566 & 0.807 & 1.102 & 1.374 \\
\hline M8.7-MTR2 & 0.297 & 0.222 & 0.371 & 0.546 & 0.799 & 1.101 & 1.375 \\
\hline M9.0-MTR1 & 0.652 & 0.488 & 0.814 & 1.226 & 1.694 & 2.299 & 2.700 \\
\hline M9.0-MTR2 & 0.601 & 0.490 & 0.775 & 1.163 & 1.713 & 2.292 & 2.680 \\
\hline M9.0-HSP1 & 0.598 & 0.435 & 0.740 & 0.897 & 1.397 & 2.179 & 2.639 \\
\hline M9.0-HSP2 & 0.606 & 0.436 & 0.747 & 0.907 & 1.426 & 2.107 & 2.517 \\
\hline$U(\mathrm{~m} / \mathrm{s})$ & $\mu_{U}$ & $\sigma_{U}$ & $U_{r m s}$ & $U_{1 / 3}$ & $U_{1 / 10}$ & $U_{1 / 100}$ & $U_{1 / 1000}$ \\
\hline M8.0-MTR1 & 0.056 & 0.109 & 0.122 & 0.115 & 0.236 & 0.892 & 2.108 \\
\hline M8.0-MTR2 & 0.038 & 0.086 & 0.094 & 0.076 & 0.134 & 0.405 & 1.731 \\
\hline M8.3-MTR1 & 0.165 & 0.184 & 0.247 & 0.319 & 0.547 & 1.417 & 2.581 \\
\hline M8.3-MTR2 & 0.159 & 0.177 & 0.238 & 0.312 & 0.533 & 1.320 & 2.554 \\
\hline M8.7-MTR1 & 0.466 & 0.340 & 0.577 & 0.841 & 1.235 & 1.863 & 2.400 \\
\hline M8.7-MTR2 & 0.441 & 0.338 & 0.556 & 0.815 & 1.209 & 1.806 & 2.149 \\
\hline M9.0-MTR1 & 0.756 & 0.473 & 0.892 & 1.280 & 1.704 & 2.571 & 5.011 \\
\hline M9.0-MTR2 & 0.814 & 0.579 & 0.999 & 1.465 & 2.051 & 3.027 & 5.009 \\
\hline M9.0-HSP1 & 0.747 & 0.549 & 0.927 & 1.127 & 1.763 & 2.749 & 3.593 \\
\hline M9.0-HSP2 & 0.819 & 0.605 & 1.019 & 1.263 & 1.908 & 2.841 & 3.516 \\
\hline$F(\mathrm{kN} / \mathrm{m})$ & $\mu_{F}$ & $\sigma_{F}$ & $F_{r m s}$ & $F_{1 / 3}$ & $F_{1 / 10}$ & $F_{1 / 100}$ & $F_{1 / 1000}$ \\
\hline M8.0-MTR1 & 0.024 & 0.199 & 0.200 & 0.063 & 0.176 & 1.295 & 4.919 \\
\hline M8.0-MTR2 & 0.010 & 0.205 & 0.206 & 0.027 & 0.067 & 0.373 & 3.033 \\
\hline M8.3-MTR1 & 0.159 & 0.375 & 0.407 & 0.397 & 0.880 & 3.280 & 4.501 \\
\hline M8.3-MTR2 & 0.134 & 0.234 & 0.270 & 0.335 & 0.695 & 1.476 & 2.749 \\
\hline M8.7-MTR1 & 1.374 & 2.209 & 2.601 & 3.360 & 6.634 & 14.065 & 23.618 \\
\hline M8.7-MTR2 & 1.285 & 2.116 & 2.476 & 3.202 & 6.447 & 13.277 & 19.043 \\
\hline M9.0-MTR1 & 3.576 & 4.636 & 5.855 & 8.307 & 15.277 & 24.486 & 36.386 \\
\hline M9.0-MTR2 & 4.051 & 5.528 & 6.853 & 9.798 & 17.688 & 30.455 & 42.936 \\
\hline M9.0-HSP1 & 3.788 & 5.796 & 6.924 & 6.722 & 14.539 & 31.737 & 55.390 \\
\hline M9.0-HSP2 & 4.424 & 6.029 & 7.477 & 7.929 & 16.093 & 30.003 & 49.653 \\
\hline
\end{tabular}

Table 7 Statistics of simulation results computed at $J=18,201$ save points along the $5 \mathrm{~m}$ isobath (excluding large bays) for LSB/ASZ sources (Table 1 Fig. 14: mean, standard deviation, root-mean-square and average of the top 33,10,1 and 0.1 percentiles of maximum elevation $\eta(\mathrm{m})$, flow velocity $U(\mathrm{~m} / \mathrm{s})$, and momentum force $F(\mathrm{kN} / \mathrm{m})$. 


\begin{tabular}{|c||c|c|c|c|c|c|c|}
\hline$\eta(\mathrm{m})$ & $\mu_{\eta}$ & $\sigma_{\eta}$ & $\eta_{r m s}$ & $\eta_{1 / 3}$ & $\eta_{1 / 10}$ & $\eta_{1 / 100}$ & $\eta_{1 / 1000}$ \\
\hline \hline M8.3-PRT1 & 0.148 & 0.108 & 0.183 & 0.274 & 0.384 & 0.561 & 0.696 \\
M8.3-PRT2 & 0.086 & 0.056 & 0.103 & 0.149 & 0.210 & 0.326 & 0.484 \\
M8.3-PRT3 & 0.046 & 0.030 & 0.055 & 0.075 & 0.102 & 0.208 & 0.410 \\
M8.7-PRT1 & 0.216 & 0.145 & 0.260 & 0.382 & 0.540 & 0.747 & 0.841 \\
M8.7-PRT2 & 0.377 & 0.225 & 0.439 & 0.659 & 0.803 & 0.855 & 0.903 \\
M9.0-PRT1 & 0.991 & 0.634 & 1.177 & 1.745 & 2.334 & 3.148 & 3.765 \\
M9.0-PRT2 & 0.800 & 0.540 & 0.965 & 1.429 & 1.969 & 2.921 & 3.947 \\
M9.0-PRT3 & 1.164 & 0.786 & 1.404 & 2.087 & 2.852 & 4.217 & 4.868 \\
\hline \hline$U$ (m/s) & $\mu_{U}$ & $\sigma_{U}$ & $U_{r m s}$ & $U_{1 / 3}$ & $U_{1 / 10}$ & $U_{1 / 100}$ & $U_{1 / 1000}$ \\
\hline \hline M8.3-PRT1 & 0.208 & 0.176 & 0.272 & 0.381 & 0.605 & 1.226 & 1.956 \\
M8.3-PRT2 & 0.128 & 0.117 & 0.174 & 0.229 & 0.374 & 0.901 & 1.807 \\
M8.3-PRT3 & 0.061 & 0.054 & 0.082 & 0.104 & 0.167 & 0.440 & 0.763 \\
M8.7-PRT1 & 0.305 & 0.226 & 0.379 & 0.534 & 0.8123 & 1.512 & 2.277 \\
M8.7-PRT2 & 0.530 & 0.341 & 0.630 & 0.904 & 1.276 & 2.035 & 2.562 \\
M9.0-PRT1 & 1.232 & 0.719 & 1.426 & 2.005 & 2.820 & 4.111 & 4.938 \\
M9.0-PRT2 & 1.120 & 0.905 & 1.440 & 2.009 & 3.188 & 6.232 & 7.688 \\
M9.0-PRT3 & 1.255 & 0.748 & 1.461 & 2.062 & 2.862 & 4.604 & 6.602 \\
\hline \hline$F$ (kN/m) & $\mu_{F}$ & $\sigma_{F}$ & $F_{r m s}$ & $F_{1 / 3}$ & $F_{1 / 10}$ & $F_{1 / 100}$ & $F_{1 / 1000}$ \\
\hline \hline M8.3-PRT1 & 0.270 & 0.504 & 0.571 & 0.654 & 1.359 & 3.946 & 6.248 \\
M8.3-PRT2 & 0.100 & 0.254 & 0.273 & 0.237 & 0.508 & 1.928 & 4.701 \\
M8.3-PRT3 & 0.025 & 0.092 & 0.095 & 0.058 & 0.132 & 0.695 & 1.700 \\
M8.7-PRT1 & 0.524 & 0.688 & 0.865 & 1.184 & 2.207 & 4.253 & 5.287 \\
M8.7-PRT2 & 1.378 & 1.252 & 1.862 & 2.886 & 4.143 & 5.1682 & 5.776 \\
M9.0-PRT1 & 9.060 & 10.937 & 14.202 & 19.289 & 34.444 & 76.255 & 107.810 \\
M9.0-PRT2 & 6.995 & 10.026 & 12.225 & 16.012 & 30.981 & 65.731 & 92.363 \\
M9.0-PRT3 & 9.914 & 10.950 & 14.771 & 21.291 & 35.827 & 63.969 & 108.922 \\
\hline
\end{tabular}

Table 8 Statistics of simulation results computed at $J=18,201$ save points along the $5 \mathrm{~m}$ isobath (excluding large bays) for PRT sources (Table 2.2 Fig. 15: mean, standard deviation, root-mean-square and average of the top 33, 10, 1 and 0.1 percentiles of maximum elevation $\eta(\mathrm{m})$, flow velocity $U(\mathrm{~m} / \mathrm{s})$, and momentum force $F(\mathrm{kN} / \mathrm{m})$.

the most part, with currents being less than 2 knots $(1 \mathrm{~m} / \mathrm{s})$ at all but a few locations (in 0.1 percentile). Note that larger current (and corresponding force) values can result here from the coarse grid resolution and insufficient energy dissipation by bottom friction nearshore. Overall, statistics show that the physical metrics for the M8 and M8.3 sources cause a low to medium-low hazard. For elevation, only $0.1 \%$ of elevation values are in a hazard class higher than 1; $0.1 \%$ of current values are in a hazard class higher than 2, and $99.9 \%$ of the force values are in hazard class 1 . For the larger ACZ sources, results in Figs. $14 \mathrm{~A}-\mathrm{c}$ and in Table 7 show that, as expected, the largest hazard for the first three metrics is caused by the four M9.0 sources, with many values falling in the high/highest hazard classes $4 / 5$ 
for each metric (Table 6). Among those, at many locations, slightly larger values of the three metrics are caused by the sources located west of the Madeira Torre Rise (MTR); but there are also locations where the sources located in the Horseshoe Plain (HSP) cause larger values. Looking at the detailed impact in the figures, South of 35.5 Lat. N. (approximately Cape Hatteras, NC), there are only small differences for the 3 metrics, between the sources located west of the MTR (M9-MTR1, M9-MTR2) and those east of it (M9-HSP1, M9HSP2), with even some locations in the south where currents and forces are slightly larger for the latter two sources, indicating that here the Madeira Torre Rise (MTR) either does not affect tsunami propagation or reinforces it slightly. In contrast, north of 35 Lat. N. the tsunami hazard for the 3 metrics is larger for the M9 sources located west of the MTR than for the HSP sources, indicating that the shallow MTR ridge offers some protection from coseismic sources located in the area of the Lisbon 1755 event. Here, while maximum surface elevations reach similar maximum values as in the south, mostly in the high hazard category, currents and forces reach values in the highest hazard category at a larger number of locations.

Regarding the medium-high magnitude M8.7 LSB/ACZ sources, results in Figs. 14a-c and in Table 7 for the three metrics show that, overall, these sources, which are about 3 times less energetic than the M9 sources (see Eq. 1), cause less impact at most locations than the M9 sources, while following the same pattern of highs and lows along the coast as for the M9 sources. However, at some isolated locations in the south, maximum elevation reaches up to $1.9 \mathrm{~m}$ for the M8.7 sources and in the north, particularly above 39 Lat. $\mathrm{N}$ (about Atlantic City, NJ), at a few locations currents and forces reach values nearly as large as those of the M9-HSP1 and M9-HSP2 sources. Clearly, this results from the complex tsunami propagation from the source, including wave interactions with the MTR near the source and with bathymetry and coastal features along the USEC shelf break and shelf. Resonances (seiching) can also be triggered nearshore due to different frequency content in the incident wave train, that may increase currents even if tsunami elevations are not significantly affected.

For PRT sources, Figs. $15 \mathrm{k}$-c show that, as a result of refraction and an overall identical northward directivity of each PRT/Caribbean arc source, the impact of each source follows the same pattern of focusing/defocusing on the same coastal areas, with just a modulation of the intensity commensurate with the source magnitude. However, the overall intensity of the impact for the 3 physical metrics is 2-3 times smaller, south of 34 Lat. N. (approximately south of Cape Hatteras, NC), than north of it. So clearly the PRT/Caribbean arc sources mostly impact the areas less impacted by the ACZ/LSB sources.

Results of the 3 physical metrics for the PRT/Caribbean arc sources in Figs. 15 $15-c$ and in Table 8 show that the three M8.3 sources, which have about 21 times less energy than 
the M9.0 sources (see Eq. 11, only cause a $\sim 0.05-0.15 \mathrm{~m}$ average maximum elevation, with a $0.03-0.011 \mathrm{~m}$ standard deviation, and $0.4-0.7 \mathrm{~m}$ for the 0.1 percentile values. The two M8.7 sources, which have 11 times less energy than the M9.0 sources, have a $\sim 0.22-$ $0.38 \mathrm{~m}$ average maximum elevation with a $0.15-0.23 \mathrm{~m}$ standard deviation, and 0.8-0.9 $\mathrm{m}$ for the 0.1 percentile values. Average maximum currents and forces for these sources are commensurate with these inundation values and small for the most part, with currents being less than $1.25 \mathrm{~m} / \mathrm{s}$ ( 2.5 knots) at all but a few locations (in 0.1 percentile). Note that larger current (and corresponding force) values can again result from the coarse grid resolution and insufficient energy dissipation by bottom friction nearshore. Overall, statistics show that the physical metrics for the M8.3 and M8.7 sources cause a low to medium-low hazard. Only $0.1 \%$ of maximum current values are in hazard class 3 . Tsunami impact is more severe for the three larger M9 PRT sources. Despite resulting from quite different fault areas, results in Figs. 15 -c and in Table 8 show fairly similar overall impacts for the three M9 sources, with moderate average values of the 3 metrics. However, there is large alongshore variation of the metrics as confirmed by values in the two highest percentile ( 1 and $0.1 \%$ ), that all fall in hazard classes $4 / 5$, i.e., high to highest impact. Among the three sources M9.0-PRT3, which was used as an extreme PMT source in earlier work, causes the maximum inundation and forces, but M9.0-PRT2 causes slightly larger maximum currents for the 0,1 and 0.1 percentile, all in the highest hazard category.

4.2 tsunami Intensity Index (TII)

To provide an overall indicator of tsunami hazard intensity along the USEC, values of the five hazard classes, computed for each source along the $5 \mathrm{~m}$ isobath for the four metrics, were combined into a Tsunami Intensity Index (TII). The TII is defined as a weighed average of the class index values, $k^{i}\left(x_{j}, y_{j}\right)=0$ to 5 (from low hazard to highest hazard), interpolated for the four metrics $M_{i}(i=1, \ldots, 4)$ (Table 6, at each save point location $\left(x_{j}, y_{j}\right)(j=1, \ldots, J)$, i.e.,

$$
\operatorname{TII}\left(x_{j}, y_{j}\right)=\sum_{i=1}^{4} w_{i} k^{i}\left(x_{j}, y_{j}\right) \quad \text { with } \quad k^{i}\left(x_{j}, y_{j}\right)=\frac{M_{i}\left(x_{j}, y_{j}\right)-C_{k, \text { min }}}{C_{k, \max }-C_{k, \text { min }}}
$$

where $w_{i}$ are weights associated with each metric, with $\sum_{i} w_{i}=1$, and the $k^{i}$ s are computed by first finding the hazard class within which each metric falls and then applying Eq. (3). The TII hence is also on a scale of 0 to 5 and represents the overall tsunami hazard intensity, from low to highest. If all metrics are given equal weight then we simply have $w_{i}=0.25$; however, as we shall see, the weights allow giving more importance to some metrics than others. 
In the following, we elected to give more weight to variables directly affecting the ability of the population to evacuate, i.e., maximum inundation height and current, and relatively less weight to forces on structures and arrival time; thus, we gave weights of $w_{i}=[0.4,0.3$, $0.15,0.15]$ to each metric, respectively. The rationale for giving less weight to arrival time is that these are far-field coseismic tsunamis for which advanced warning would be immediately given upon the triggering of the event by an earthquake, hence somewhat mitigating this part of the hazard. However, any other combination of metric weights could be applied and results easily generated. Figures 16 and 17 show the longshore distributions of TII values computed with these weights using Eq. (3), based on the four hazard metric results computed along the $5 \mathrm{~m}$ isobath for the LSB/ACZ (Fig. 14) and PRT/Caribbean arc (Fig. 15) sources, respectively. We see that the TII class values (which are color coded for different levels of hazard using the same color scheme as that of the hazard intensity classes) well summarize overall tsunami hazard and confirm the detailed observations made above on the individual hazard metrics results. The TII, however, provides a unique and simple indicator of the hazard intensity expected for each source at the many save points along the coast, that can be easily understood and used by emergency managers. Although these figures are plotted at large scale, all results produced can be easily zoomed into and/or imported into a GIS, to evaluate the hazard faced by any particular area or specific community.

\subsection{Estimating the effect of return period}

Although the complete PTHA of tsunami hazard along the USEC will be addressed in future work, the approximate return periods provided in Tables 2 and 3 for each of the 18 coseismic sources whose impact was modeled along the USEC, allow drawing some preliminary conclusions as of the expected intensity of tsunami hazard for different return periods. Specifically, the values of the TII and four associated hazard metrics, computed for each source along the coast, provide a measure of the intensity of the tsunami hazard as a function of the event return period.

Altogether, the sources simulated here cover an estimated range of return periods, $T_{r} \in$ $[70-2,130]$ years, with the low end being the M8 ACZ sources and the high end the M9.0PRT3 extreme source. While detailed results of each tsunami metric can be extracted for any save point along the coast, here, we only consider the overall statistics of the three physical tsunami metrics, maximum elevation, current and force given in Tables 7 and 8 to estimate ranges of expected values as a function of $T_{r}$. Based on these statistics, for instance, we see that the expected 0.1 percentile values up to return periods of $\sim 100$ years are a maximum inundation $\eta_{1 / 1000} \sim 0.7 \mathrm{~m}$, maximum current $U_{1 / 1000} \sim 2 \mathrm{~m} / \mathrm{s}$, and maximum force $F_{1 / 1000} \sim 5 \mathrm{kN} / \mathrm{m}$; up to $\sim 250$ years, these values become $1.4 \mathrm{~m}, 2.4 \mathrm{~m} / \mathrm{s}$ and $24 \mathrm{kN} / \mathrm{m}$; 
up to $\sim 500$ years, they become $2.6 \mathrm{~m}, 5.0 \mathrm{~m} / \mathrm{s}$ and $55 \mathrm{kN} / \mathrm{m}$; and up to $\sim 1,000$ years, they become $4 \mathrm{~m}, 7.7 \mathrm{~m} / \mathrm{s}$ and $108 \mathrm{kN} / \mathrm{m}$. Finally, up to $\sim 2,000$ years, maximum inundation approaches $5 \mathrm{~m}$, while the other metrics stay at the same level.

\section{Conclusions}

We presented the methodology for and overall results of model simulations conducted to simulate tsunami hazard along the USEC from a collection of 18 far-field coseismic sources ranging in magnitude from M8 to M9. These include 10 sources located in the ACZ area, ranging from M8 to M9 magnitude, with some representing Lisbon 1755 proxy (repeat) events and 8 sources in the PRT/Caribbean arc area from M8.3 to M9 magnitude. Results provide a comprehensive picture at the regional scale (i.e., at a coarse grid resolution) of the intensity of coastal tsunami hazard expected from each source in terms of 4 hazard metrics computed along the 5 meter isobath: (1) the maximum tsunami elevation, (2) current and (3) force, as well as tsunami arrival time. These metrics are combined into a single Tsunami Intensity Index (TII), whose value allows identifying areas and infrastructure at greater hazard from coseismic tsunamis.

The 18 sources have estimated return periods ranging approximately between 100 and 2,000 years. For each source, the TII thus provides an overall tsunami hazard intensity along the USEC coast that allows both a comparison among sources and a quantification of tsunami hazard as a function of the source return period. The detailed values of each metric, both saved over the model grids and along the $5 \mathrm{~m}$ isobath, can help quantify tsunami hazard at any given coastal location in the 14 considered US coastal states, from Florida to Maine. These can also provide initial and boundary conditions to perform higher resolution inundation mapping simulations in areas identified as having a higher tsunami hazard.

While earlier work only focused on the most extreme tsunami sources causing PMTs, whose return period was largely unknown, the present work covers a collection of sources to which approximate return periods are associated. Combined with other tsunami sources such as nearshore SMFs and far-field volcanic collapse into a logic tree, the 18 coseismic sources modeled here could thus contribute to future PTHA work along the USEC. 

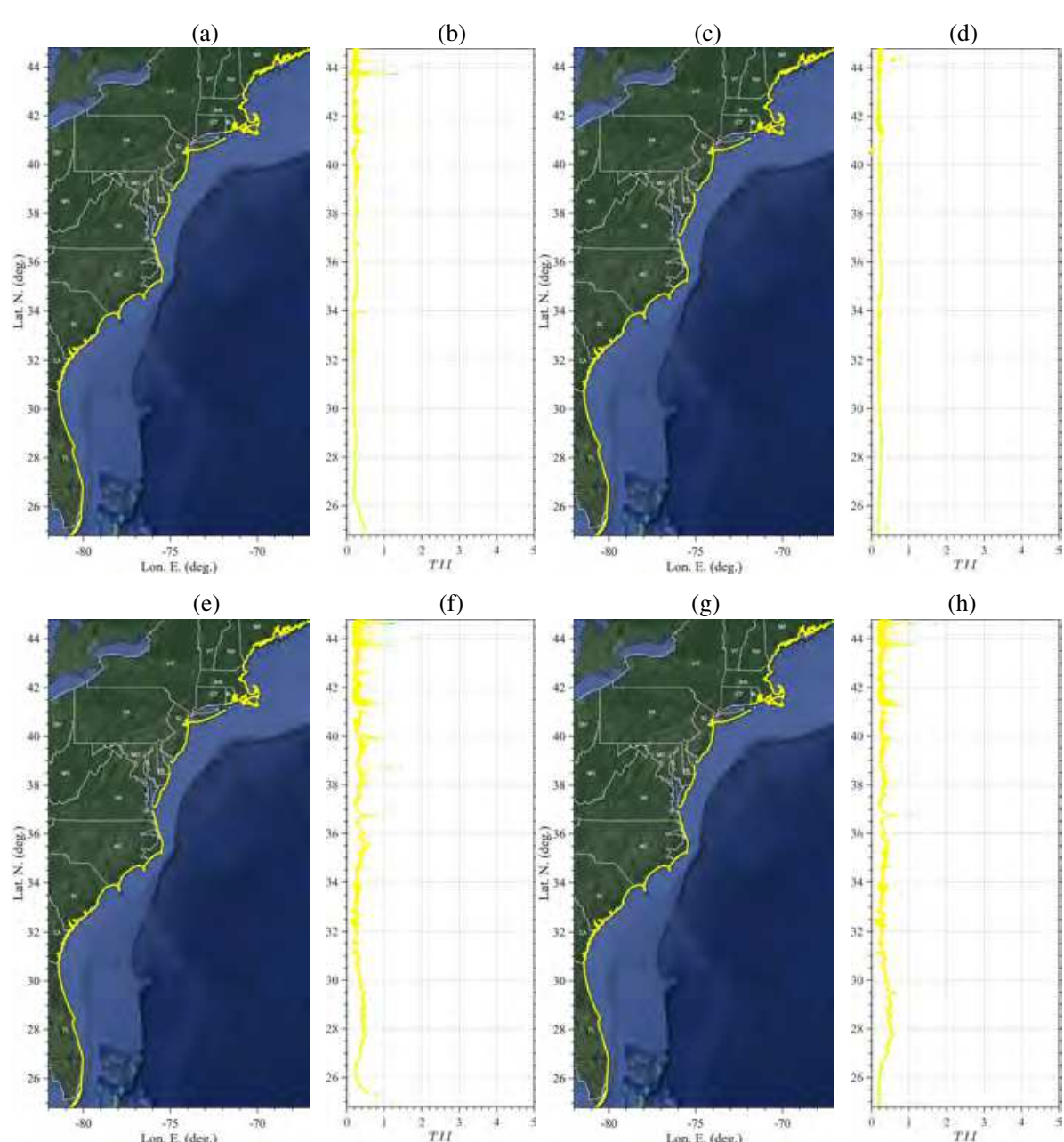

(i)

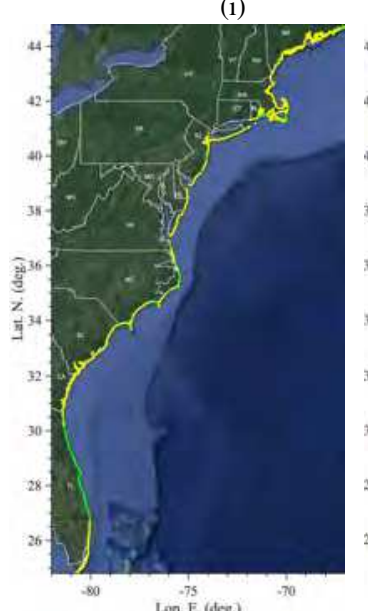

(j)

(k)
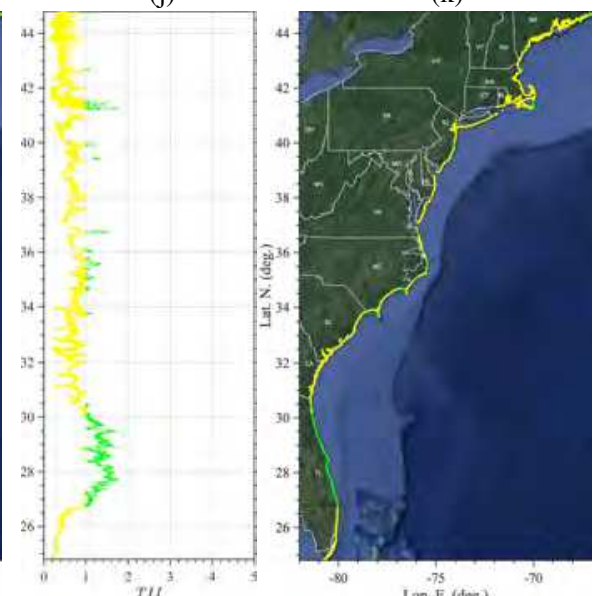

(1)

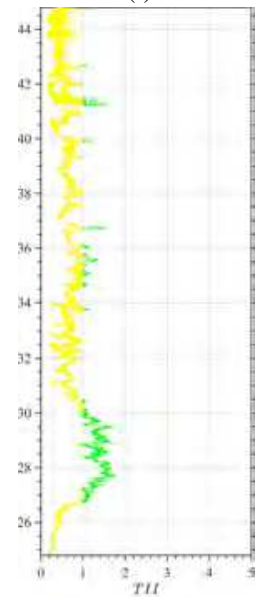




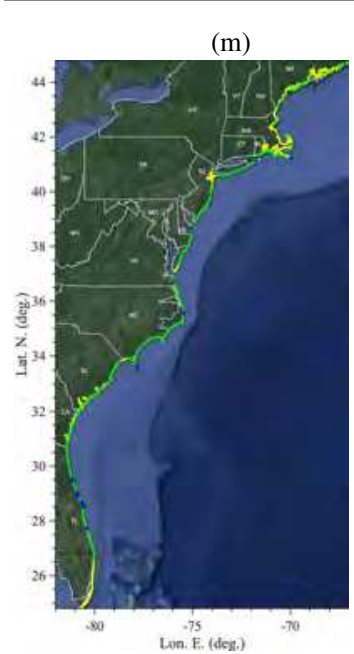

(q)

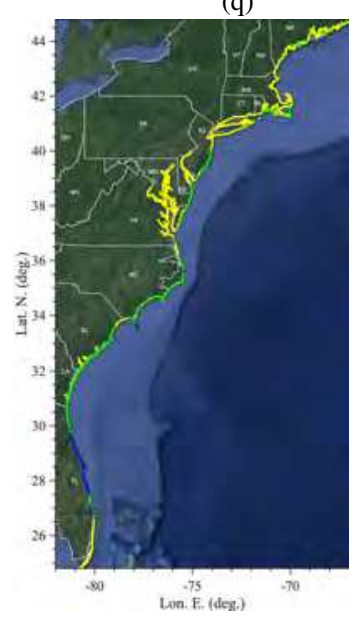

(n)

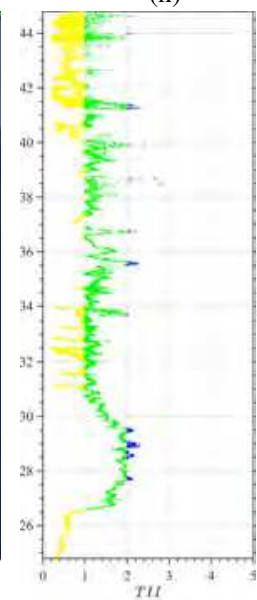

(r)

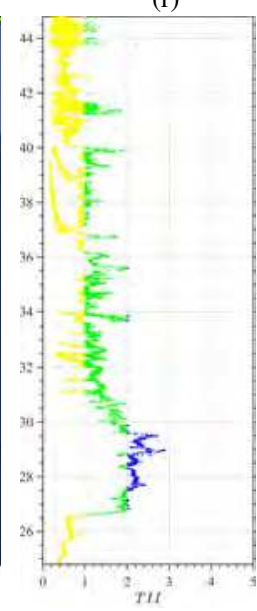

(o)

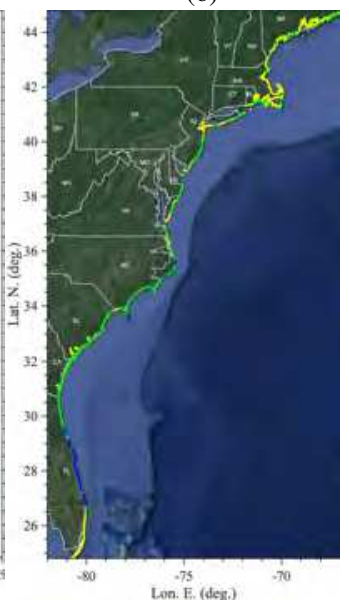

(s)

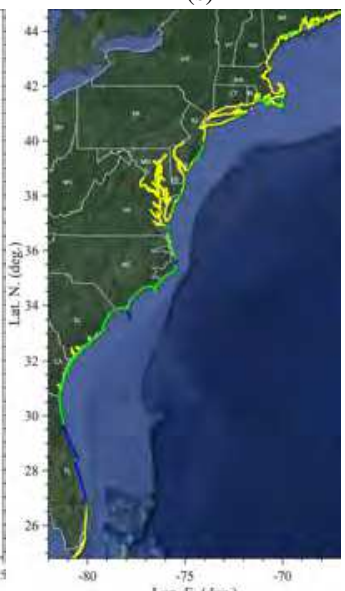

(p)

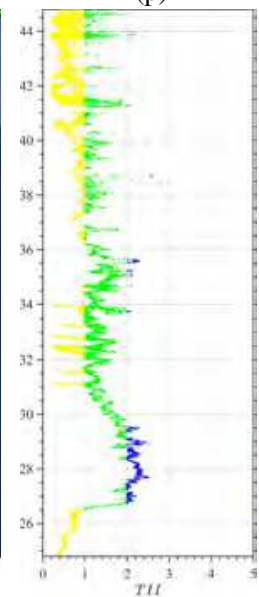

(t)

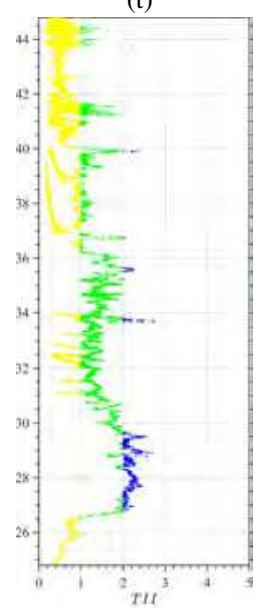

Fig. 16 Tsunami intensity index computed for ten LSB/ACZ sources (Table 1. Fig. 44, based on results of the 4 hazard metrics in Fig. 14 (a,b) M8.0-MTR1; (c,d) M8.0-MTR2; (e,f) M8.3-MTR1; (g,h) M8.3-MTR2; (i,j) M8.7-MTR1; (k,l) M8.7-MTR2; (m,n) M9.0-MTR1; (o,p) M9.0-MTR2; (q,r) M9.0-HSP1; (s,t) M9.0-HSP2. TII metric weights are $w_{i}=[0.40,0.30,0.15,0.15]$, and color coded hazard intensity classes are: (yellow) low; (green) medium low; (blue) medium (magenta) medium high, (red) heigh, and (black) extreme. Note, for the last 2 sources, the TII is also provided within the large bays.

However, pending such PTHA work, the overall estimates made here of tsunami hazard intensity for several physical variables, as a function of the event return period, can help put in perspective the tsunami hazard faced by the USEC with that resulting from other more prominent natural disasters such as tropical cyclones. For the highest hazard areas (0.1 percentile), we find that tsunami hazard in the 100-500 year return period range is commensurate with that posed by category 3-5 tropical cyclones, taking into account the 

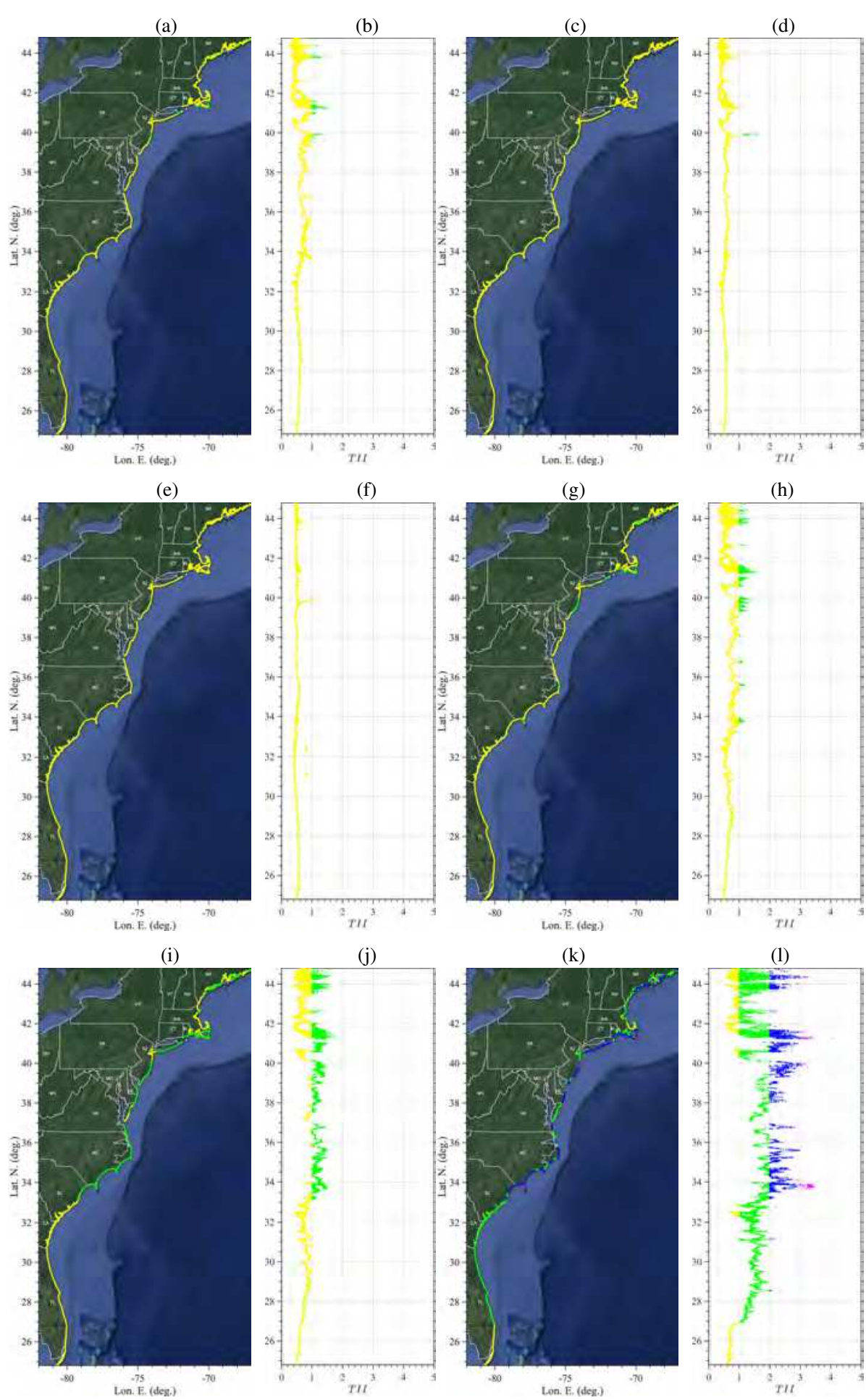


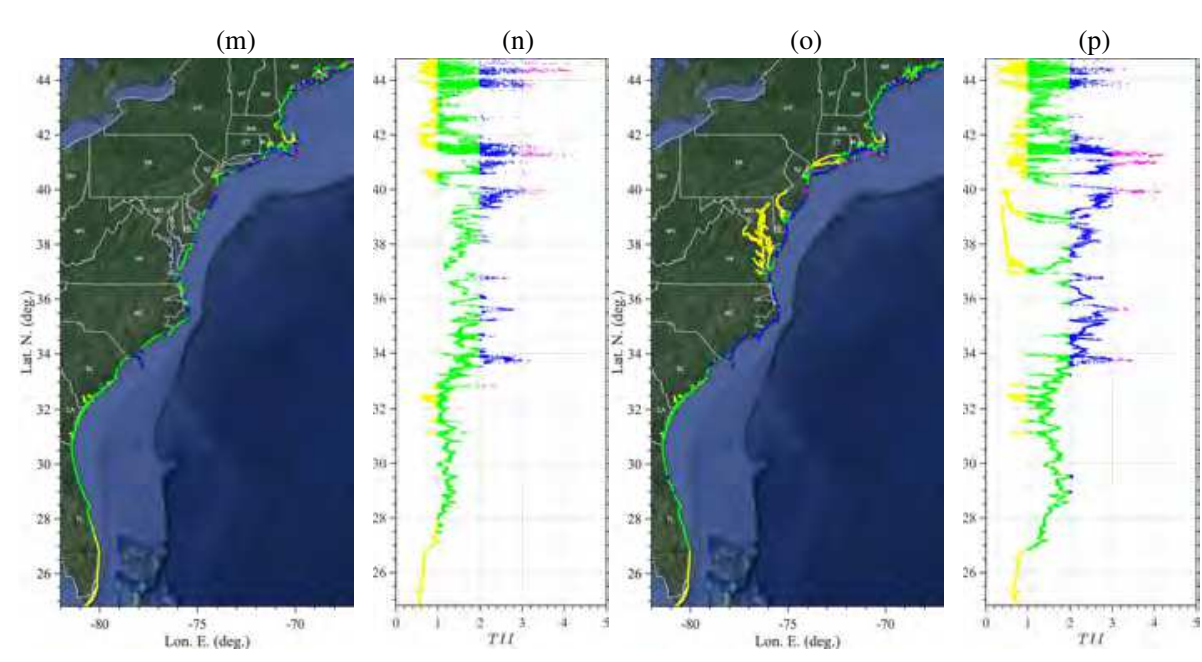

Fig. 17 Tsunami intensity index computed for eight PRT/Caribbean arc sources (Table 3; Fig. 5 , based on results of the 4 hazard metrics in Fig.15 (a,b) M8.3-PRT1; (c,d) M8.3-PRT2; (e,f) M8.3-PRT3; (g,h) M8.7PRT1; (i,j) M8.7-PRT2; (k,1) M9.0-PRT1; (m,n) M9.0-PRT2; (o,p) M9.0-PRT3. TII metric weights are $w_{i}=$ $[0.40,0.30,0.15,0.15]$, and color coded hazard intensity classes are: (yellow) low; (green) medium low; (blue) medium (magenta) medium high, (red) heigh, and (black) extreme. Note, for the last source, the TII is also provided within the large bays.

larger current velocities and forces caused by tsunami waves. Indeed, while the storm surge caused by category 3-5 hurricanes can reach 4-6 m along the USEC [58], the resulting coastal inundation is typically not associated with flow velocity and momentum forces as large as those caused by the long tsunami waves which, hence, could be more damaging even when associated with a smaller coastal inundation.

Results of our work are made available to USEC emergency managers, who will be able to look at the most exposed areas to tsunami hazard and put the associated risk in perspective with that resulting from other natural disasters and implement the necessary mitigation measures.

\section{Declarations}

\subsection{Funding}

This work was supported in the United States by NOAA's National Weather Service (NWS), through grants administered by the US National Tsunami Hazards Mitigation Program (NTHMP): NA-13-NWS4670014, NA-14-NWS4670041, NA-15-NWS4670029, NA-16-NWS4670034, NA-17-NWS4670010, NA-18-NWS4670073 and NA-19-NWS4670074. Numerical simulations reported in this work used HPC resources, as part of the Extreme Science and Engi- 
neering Discovery Environment (XSEDE) (project BCS-170015), which is supported by the National Science Foundation (NSF) grant number ACI-1548562.

6.2 Conflicts of interest/Competing interests

None

6.3 Availability of data and material (data transparency)

A Google Drive repository for all the raw data that was used to set up the tsunami models and model results has been created. This is in fact a requirement of the NTHMP program. The Google Drive will be finalize and made public once the paper is accepted for publication. Note that the raw data does not provide any useful information in itself regarding.

6.4 Code availability

FUNWAVE-TVD, the tsunami model used in this work, is open source software available at http://github.com/fengyanshi/FUNWAVE-TVD/.

6.5 Authors' contributions

SG: Led work, acquired funding, defined methodology, processed results and wrote most of the paper.

MM: Performed model simulations, did background literature work, post processed results, made some figures and proofread paper.

LS: Created model grids and performed model simulations for a few extreme sources, did background literature work, developed post-processing routines, and did some post processing of results.

AG: Co-led work, helps to acquire funding, defined some of the methodology, performed early tsunami simulations and developed initial post-processing routines, proofread paper.

6.6 Ethics approval

Not applicable. 
6.7 Consent to participate

Not applicable.

\subsection{Consent for publication}

Not applicable

Acknowledgements The authors gratefully acknowledge continuing support from NOAA's National Weather Service (NWS), through grants administered by the US National Tsunami Hazards Mitigation Program (NTHMP): NA-13-NWS4670014, NA-14-NWS4670041, NA-15-NWS4670029, NA-16-NWS4670034, NA17-NWS4670010, NA-18-NWS4670073 and NA-19-NWS4670074. Numerical simulations reported in this work used HPC resources, as part of the Extreme Science and Engineering Discovery Environment (XSEDE) (project BCS-170015), which is supported by the National Science Foundation (NSF) grant number ACI1548562. FUNWAVE-TVD is open source software available at http://github.com/fengyanshi/FUNWAVE$\mathrm{TVD} /$.

\section{References}

1. Abadie S., Harris J.C., Grilli S.T. and R. Fabre, Numerical modeling of tsunami waves generated by the flank collapse of the Cumbre Vieja Volcano (La Palma, Canary Islands): tsunami source and near field effects, Journal of Geophysical Research, 117, C05030, doi:10.1029/2011JC007646 (2012)

2. Baptista, M. A., Heitor, S., Miranda, J.M., Miranda, P.M.A. and Mendes Victor, L., The 1755 Lisbon earthquake; evaluation of the tsunami parameters, J. Geodynamics, 25, 143-157 (1998a)

3. Baptista, M. A., Heitor, S., Miranda, J.M., Miranda, P.M.A. and Mendes Victor, L., Constraints on the source of the 1755 Lisbon tsunami inferred from numerical modeling of historical data on the source of the 1755 Lisbon tsunami, J. Geodynamics, 25, 159-174 (1998b)

4. Baptista, M.A., Miranda, J. M., Chierici, F. and Zitellini, N., New study of the 1755 earthquake source based on multi-channel seismic survey data and tsunami modeling, Nat. Haz. Earth Syst. Sc., 3, 333-340 (2003)

5. Baptista, M. A. And Miranda, J. M., Revision of the Portuguese catalog of tsunamis. Natural Hazards and Earth System Sciences, 9(1), 25-42 (2009)

6. Baptista, M. A., Miranda, J. M., Batlló, J., Lisboa, F., Luis, J., and Macia?, R., New study on the 1941 Gloria Fault earthquake and tsunami. Natural Hazards and Earth System Sciences, 16(8), 1967-1977 (2016)

7. Baptista, M.A., 2020, Tsunamis Along the Azores Gibraltar Plate Boundary. Pure and Applied Geophysics, 177, 1713-1724 (2020)

8. Barkan, R., ten Brick, U.S. and Lin, J., Far field tsunami simulations of the 1755 Lisbon earthquake: Implication for tsunami hazard to the U.S. East Coast and the Caribbean, Marine Geology, 264, 109-122 (2009)

9. Benford, B., DeMets, C., Calais, E. GPS estimates of microplate motions, northern Caribbean: evidence for a Hispaniola microplate and implications for earthquake hazard. Geophys. J. Intl., 191(2), 481-490, doi:10.1111/j.1365-246X.2012.05662.x (2012) 
10. Boschetti L., Ioualalen M., Nemati F., Grilli S.T., Dessa J.-X., and C. Larroque, Tsunami intensity scale based on wave amplitude and current applied to the French Riviera: the case study of local seismicity. Nat. Hazards, 102, 219-248, doi: 10.1007/s11069-020-03921-0 (2020)

11. Calais, E. And Mercier de Lepinay. B. Strike-slip tectonic processes in the northern Caribbean between Cuba and Hispaniola (Windward Passage). Mar. Geophys. Res., 17(1), 63-95 (1995)

12. Calais, E., Freed, A., Mattioli, G., Amelung, F., Jónsson, S., Jansma, P., Hong, S-H., Dixon, T., Prépetit, C. and Momplaisir, R. Transpressional rupture of an unmapped fault during the 2010 Haiti earthquake. Nat. Geoscience, 3(11), 749-799, doi:10.1038/NGEO992 (2010)

13. Chaytor J.D., ten Brink U.S., Solow A.R., and B.D. Andrews, Size distribution of submarine landslides along the U.S. Atlantic margin. Marine Geology, 264(1-2):16-27 (2009)

14. Dawicki, S., Major Caribbean Earthquakes and Tsunamis a Real Risk: Events rare, but scientists call for public awareness, warning system, Media Relations, Woods Hole Oceanographic Institute, available at: http://www.whoi.edu/page.do?pid=12460\&tid=282\&cid=2430 (last access: 13 December 2008 (2005)

15. DeMets, C., Earthquake slip vectors and estimates of present-day plate motions. J. Geophys. Res., 98 , 6703-6714 (1993).

16. Gailler, A., Calais, E., Hébert, H., Roy, C., Okal, E. Tsunami scenarios and hazard assessment along the northern coast of Haiti. Geophys. J. Intl., 203(3), 2287-2302, doi:10.1093/gji/ggv428 (2015)

17. Gica, E., M. C. Spillane, V. V. Titov, C. D. Chamberlin, and J. Newman, Development of the forecast propagation database for NOAA's Short-Term Inundation Forecast for Tsunamis, NOAA Tech. Memo., OAR PMEL-139 (2008)

18. Glimsdal S., Pedersen G.K., Harbitz C.B., and F. Løvholt, Dispersion of tsunamis: does it really matter ?, Nat. Hazards Earth Syst. Sci., 13, 1507-1526, doi:10.5194/nhess-13-1507-2013 (2013)

19. Grilli A.R. and S.T. Grilli, Modeling of tsunami generation, propagation and regional impact along the U.S. East Coast from the Azores Convergence Zone, Research Report no. CACR-13-04, 20 pps., http://personal.egr.uri.edu/grilli//grilli-grilli-cacr-13-04 (2013a)

20. Grilli A.R. and S.T. Grilli, Modeling of tsunami generation, propagation and regional impact along the upper U.S East coast from the Puerto Rico trench, Research Report no. CACR-13-02, 18 pps., http://personal.egr.uri.edu/grilli//grilli-grilli-cacr-13-02 (2013b)

21. Grilli, S.T., Grilli A.R., David, E. and C. Coulet, Tsunami Hazard Assessment along the North Shore of Hispaniola from far- and near-field Atlantic sources. Natural Hazards, 82(2), 777-810, doi: 10.1007/s11069-016-2218-z (2016)

22. Grilli S.T., Ioualalen M., Asavanant J., Shi F., Kirby J.T., and P. Watts, Source constraints and model simulation of the December 26, 2004 Indian Ocean tsunami, Journal of Waterway, Port, Coastal, and Ocean Engineering, 33, 414-428 (2007)

23. Grilli S.T., Dubosq S., Pophet N., Pérignon Y., Kirby J.T., and F. Shi, Numerical simulation and firstorder hazard analysis of large coseismic tsunamis generated in the Puerto Rico thrench: near-field impact on the North shore of Puerto Rico and far-field impact on the US East Coast, Natural Hazards and Earth System Sciences, 10, 2109-2125, doi:10.5194/nhess-2109-2010 (2010)

24. Grilli, S.T., J.C. Harris, T. Tajalibakhsh, T.L. Masterlark, C. Kyriakopoulos, J.T., Kirby and F. Shi Numerical simulation of the 2011 Tohoku tsunami based on a new transient FEM coseismic source: Comparison to far- and near-field observations, Pure Appl. Geophys., 170, 1333-1359, doi:10.1007/s00024-012-0528-y (2013c)

25. Grilli S.T., O’Reilly C., Harris J.C., Tajalli-Bakhsh T., Tehranirad B., Banihashemi S., Kirby J.T., Baxter C.D.P., Eggeling T., Ma G. and F. Shi, Modeling of SMF tsunami hazard along the upper US East Coast: Detailed impact around Ocean City, MD, Natural Hazards, 76(2), 705-746, doi: 10.1007/s11069-014$1522-8(2015)$ 
26. Grilli S.T., D.R. Tappin, S. Carey, S.F.L. Watt, S.N. Ward, A.R. Grilli, S.L. Engwell, C. Zhang, J.T. Kirby, L. Schambach and M. Muin, Modelling of the tsunami from the December 22, 2018 lateral collapse of Anak Krakatau volcano in the Sunda Straits, Indonesia, Scientific Reports, 9, 11946, doi:10.1038/s41598-01948327-6 (2019)

27. Grilli, S.T., Shelby, M., Kimmoun, O., Dupont, G., Nicolsky, D., Ma, G., Kirby, J. and F. Shi, Modeling coastal tsunami hazard from submarine mass failures: effect of slide rheology, experimental validation, and case studies off the US East coast, Natural Hazards, 86(1), 353-391, doi:10.1007/s11069-016-2692-3 (2017)

28. Harbitz, C.B., Glimsdal S., Bazin S., Zamora N., Løvholt F., Bungum H., Smebye H., Gauer P., and Kjekstad O., Tsunami hazard in the Caribbean: Regional exposure derived from credible worst case scenarios, Continental Shelf Res., 8, 1-23, doi:10.1016/j.csr.2012.02.006 (2012)

29. Horrillo J., Grilli S.T., Nicolsky D., Roeber V., and J. Zhang, Performance bench- marking tsunami models for NTHMP's inundation mapping activities, Pure and Applied Geophysics, 172(3-4), 869-884 (2015)

30. Ioualalen M., Asavanant J., Kaewbanjak N., Grilli S.T., Kirby J.T. and P. Watts, Modeling the 26th December 2004 Indian Ocean tsunami: Case study of impact in Thailand, J. Geophys. Res., 112, C07024, doi:10.1029/2006JC003850 (2007)

31. Kirby J.T., Shi F., Tehranirad B., Harris J.C. and S.T. Grilli, Dispersive tsunami waves in the ocean: Model equations and sensitivity to dispersion and Coriolis effects, Ocean Modell., 62, 39-55, doi:10.1016/j.ocemod.2012.11.009 (2013)

32. Knight, B., Model predictions of Gulf and Atlantic Coast tsunami impacts from a distribution of sources, Science of Tsunami Hazard, 24(5), 304-312 (2006)

33. Lander, J.F., Caribbean Tsunamis: an initial history, Nat. Haz., 3, 1-18 (1997)

34. Lockridge, P.A., Whiteside, L.S. And J.F. Lander, Tsunamis and tsunami-like waves of the eastern United States, Science of Tsunami Hazards, 20(3), 120-157 (2002).

35. López-Venegas, A.M., Horrillo, J., Pampell-Manis, A., Huérfano, V., Mercado, A. Advanced tsunami numerical simulations and energy considerations by use of 3D-2D coupled models: the October 11, 1918 Mona passage tsunami. Pure Appl. Geophys., 172(6), 1679-1698, doi:10.1007/s00024-014-0988-3 (2015). 36. Løvholt, F., Pedersen, G. And G. Gisler, Oceanic propagation of a potential tsunami from the La Palma Island, Journal of Geophysical Research: Oceans, 113(C9) (2008)

37. Løvholt, F., Schulten, I., Mosher, D., Harbitz, C. And S. Krastel, Modelling the 1929 Grand Banks slump and landslide tsunami, Geological Society, London, Special Publications, 477(1), 315-331, https://doi.org/10.1144/SP477.28 (2019)

38. Lynett, P., and 37 alii, Inter-Model Analysis of Tsunami-Induced Coastal Currents, Ocean Modell., 114, 14-32, doi:10.1016/j.ocemod.2017.04.003 (2017)

39. Madsen P.A., D.R. Fuhrman and H. A. Schaffer, On the solitary wave paradigm for tsunamis. J. Geophys. Res., 113, C12012 (2008)

40. Mercado, A. And W. McCann, Numerical simulation of the 1918 Puerto Rico tsunami, Natural Hazards, 18(1), 57-76 (1998)

41. Muir-Wood, R. And A. Mignan, A phenomenological reconstruction of the Mw9 November 1 st 1755 earthquake source, In The 1755 Lisbon Earthquake: Revisited, pp. 121-146, Springer, Dordrecht, (2009)

42. Nemati F., Grilli S.T., Ioualalen M., Boschetti L., Larroque L. and J. Trevisan, High-resolution coastal hazard assessment along the French Riviera from coseismic tsunamis generated in the Ligurian fault system. Nat. Hazards, 96(2), 553-586, doi:10.1007/s11069-018-3555-x (2019)

43. Okada, Y., Surface deformation due to shear and tensile faults in a half space, Bull. Seismol. Soc. America, 75(4), 1135-1154 (1985) 
44. Omira, R., Baptista, M. A., Matias, L., Miranda, J. M., Catita, C., Carrilho, F., et al., Design of a sealevel tsunami detection network for the Gulf of Cadiz. Natural Hazards and Earth System Sciences, 9(4), $1327 ? 1338$ (2009)

45. Schambach L., Grilli S.T., Kirby J.T. and F. Shi Landslide tsunami hazard along the upper US East Coast: effects of slide rheology, bottom friction, and frequency dispersion, Pure Appl. Geophys., 176(7), 3,059-3,098, doi:10.1007/s00024-018-1978-7 (2019)

46. Schambach L., Grilli S.T., Tappin D.R., Gangemi M.D., and G. Barbaro, New simulations and understanding of the 1908 Messina tsunami for a dual seismic and deep submarine mass failure source, Marine Geology, 421, 106093, doi: 10.1016/j.margeo.2019.106093 (2020)

47. Schambach L., Grilli S.T., Tappin D.R., New high-resolution modeling of the 2018 Palu tsunami, based on supershear earthquake mechanisms and mapped coastal landslides, supports a dual source, Frontiers in Earth Sciences, 8, 627, doi:0.3389/feart.2020.598839 (2020)

48. Schambach, L., Grilli, S.T. and A.R. Grilli. Simulation of Global Tsunami Hazard along the U.S. East Coast. Research Report no. CACR-20-02. NTHMP Award, \#NA18NWS4670073, National Weather Service Program Office, 93 pps., http://personal.egr.uri.edu/grilli/EC_hazard_report_NTHMP_CACRo.pdf (2020).

49. Schnyder J.S., Eberli G. P., Kirby J.T., Shi F., Tehranirad B., Mulder T., Ducassou E., Hebbeln D., and Wintersteller P., Tsunamis caused by submarine slope failures along western Great Bahama Bank. Scientific Reports, 6, 35925 (2016)

50. Shi F., Kirby J.T., Harris J.C., Geiman J.D., and S.T. Grilli, A high-order adaptive time-stepping TVD solver for Boussinesq modeling of breaking waves and coastal inundation, Ocean Modell., 43-44, 36-51, doi:10.1016/j.ocemod.2011.12.004 (2012)

51. Tappin D.R., Watts P. and S.T. Grilli, The Papua New Guinea tsunami of 1998: anatomy of a catastrophic event, Natural Hazards and Earth System Sciences, 8, 243-266 (2008)

52. Tappin D.R., Grilli S.T., Harris J.C., Geller R.J., Masterlark T., Kirby J.T., F. Shi, G. Ma, K.K.S. Thingbaijamg, and P.M. Maig (2014) Did a submarine landslide contribute to the 2011 Tohoku tsunami ? Marine Geology, 357:344-361 doi: 10.1016/j.margeo.2014.09.043

53. Tehranirad B., Shi F., Kirby, J.T., Harris J.C. and S.T. Grilli, Tsunami benchmark results for fully nonlinear Boussinesq wave model FUNWAVE-TVD, Version 1.0, Technical report, No. CACR-11-02, Center for Applied Coastal Research, University of Delaware (2011)

54. Tehranirad B., Harris J.C., Grilli A.R., Grilli S.T., Abadie S., Kirby J.T. and F. Shi, Far-field tsunami hazard in the north Atlantic basin from large scale flank collapses of the Cumbre Vieja volcano, La Palma, Pure Appl. Geophys., 172(12), 3,589-3,616, doi:10.1007/s00024-015-1135-5 (2015)

55. ten Brink, U., Twichell D., Geist E., Chaytor J., Locat J., Lee H., Buczkowski B., Barkan R., Solow A., Andrews B., Parsons T., Lynett P., Lin J., and M. Sansoucy, Evaluation of tsunami sources with the potential to impact the U.S. Atlantic and Gulf coasts, USGS Administrative report to the U.S. Nuclear Regulatory Commission, 300 pp (2008)

56. ten Brink, U.S., Bakun W.H. and Flores C.H., Historical perspective on seismic hazard to Hispaniola and the northeast Caribbean region, J. Geophys. Res. (Solid Earth), 106(B12) (2011)

57. ten Brink, U.S., Chaytor, J.D., Geist, E.L., Brothers, D.S. and B.D. Andrews, Assessment of tsunami hazard to the US Atlantic margin, Marine Geology, 353, 31-54 (2014)

58. Torres M.J., Hashemi, M.R., Hayward S., Spaulding M.L., Ginis I. and S.T. Grilli. Role of hurricane wind models in accurate simulation of storm surge and waves. J. Waterways, Port, Coastal Engng., 145(1), 04018039 (2019)

59. Udias, A., Large Earthquakes and Tsunamis at Saint Vincent Cape before the Lisbon 1755 Earthquake: A Historical Review. Pure and Applied Geophysics, 177, 1739-1745 (2020) 
60. Watts P., Grilli S.T., Kirby J.T., Fryer G.J. and D.R. Tappin, Landslide tsunami case studies using a Boussinesq model and a fully nonlinear tsunami generation model, Natural Hazards and Earth System Sciences, 3, 391-402 (2003)

61. Wei G., Kirby J.T., Grilli S.T. and R. Subramanya, A fully nonlinear Boussinesq model for free surface waves. Part I: Highly nonlinear unsteady waves, Journal of Fluid Mechanics, 294, 71-92 (1995)

62. Zahibo, N. and Pelinovsky, E. N., Evaluation of tsunami risk in the Lesser Antilles, Nat. Hazards Earth Syst. Sci., 1, 221-231, doi:10.5194/nhess-1-221-2001 (2001) 


\section{Figures}

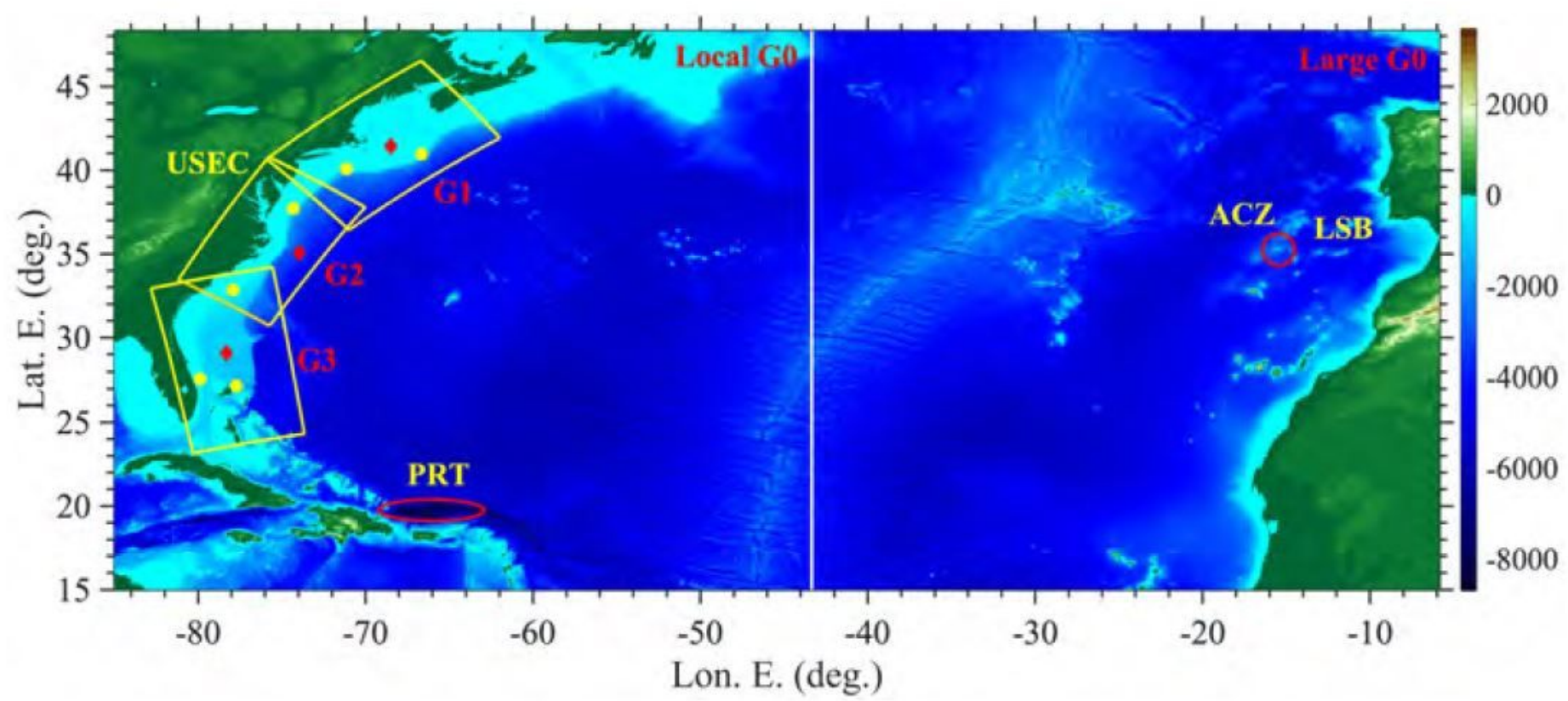

\section{Figure 1}

Footprint and ETOPO1 bathymetry/topography (color scale in meter shows $<>0$ ) of FUNWAVE's 1 arc-min resolution grids in North Atlantic Ocean basin (Local/Large G0), with footprints of 3 regional $450 \mathrm{~m}$ nested shore-parallel Cartesian grids (G1, G2, G3; Table 4). Location are marked for the two areas of historical/hypothetical tsunami coseismic sources (red oval) considered, near the Ac, ores Convergence Zone (ACZ), including Lisbon 1755 (LSB), and near and around the Puerto Rico Trench (PRT). The Madeira Torre Rise (MTR) is the shallower ridge located on the north of the ACZ circled area and the Horseshoe Plain is to the East of the ACZ and MTR. Yellow/red symbols within the regional grids mark locations of numerical wave gauge stations where time series of surface elevation are calculated in simulations for validating the one-way grid coupling (Table 2). Note: The designations employed and the presentation of the material on this map do not imply the expression of any opinion whatsoever on the part of Research Square concerning the legal status of any country, territory, city or area or of its authorities, or concerning the delimitation of its frontiers or boundaries. This map has been provided by the authors. 


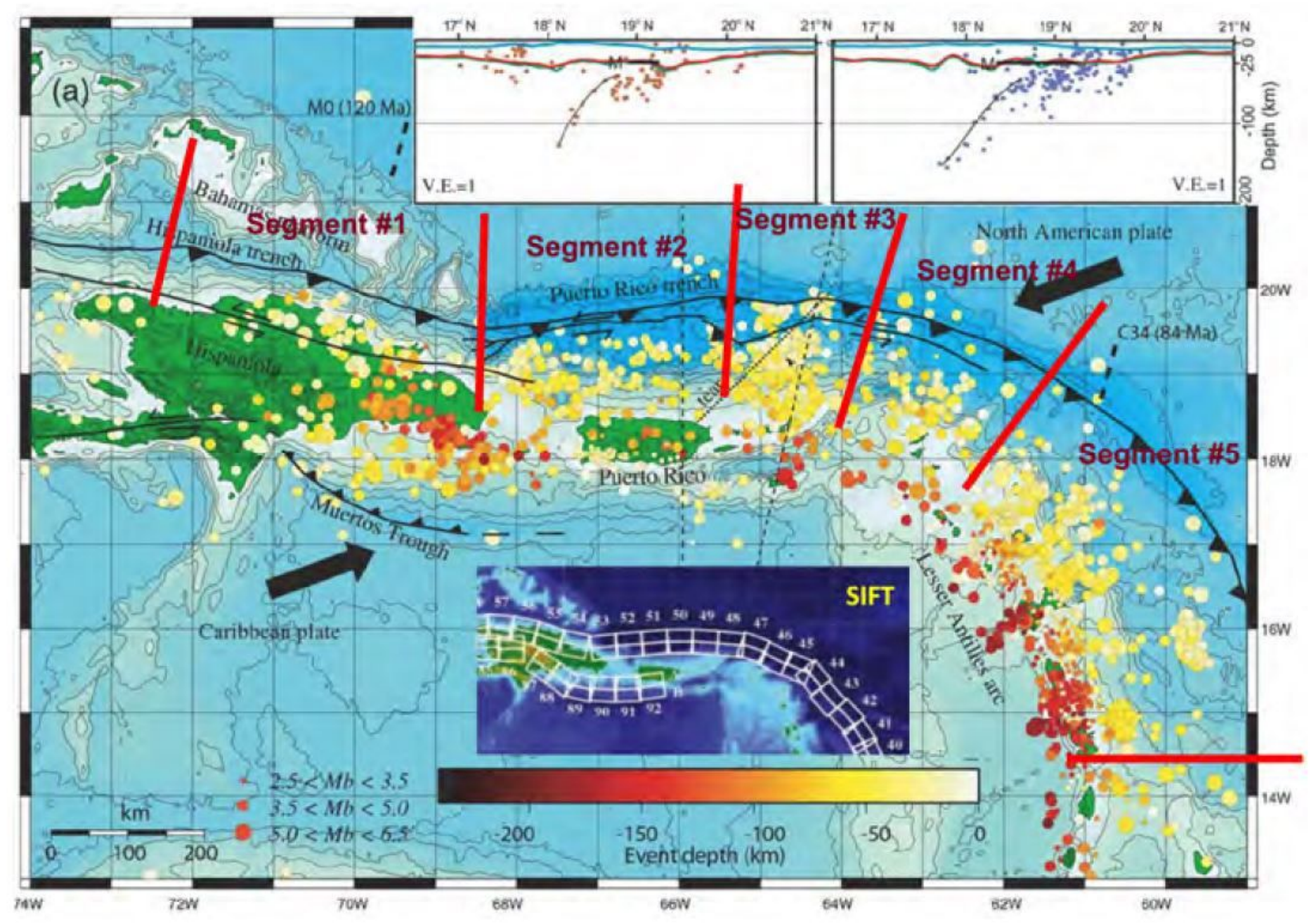

\section{Figure 2}

Likeliest segmentation in 5 segments of the PRT/Caribbean arc, from west of Hispaniola to Guadeloupe in the eastern part, established at the May 2019 workshop of experts at the USGS Powell Center (see Fig. 1 for location). Insert shows footprints of SIFT subfaults [17] in the considered area (see Table 2 for parameter values). Large black arrows show the nearly E-W relative plate motion of the North America Plate subducting under the Caribbean Plate. Note: The designations employed and the presentation of the material on this map do not imply the expression of any opinion whatsoever on the part of Research Square concerning the legal status of any country, territory, city or area or of its authorities, or concerning the delimitation of its frontiers or boundaries. This map has been provided by the authors. 
(a)
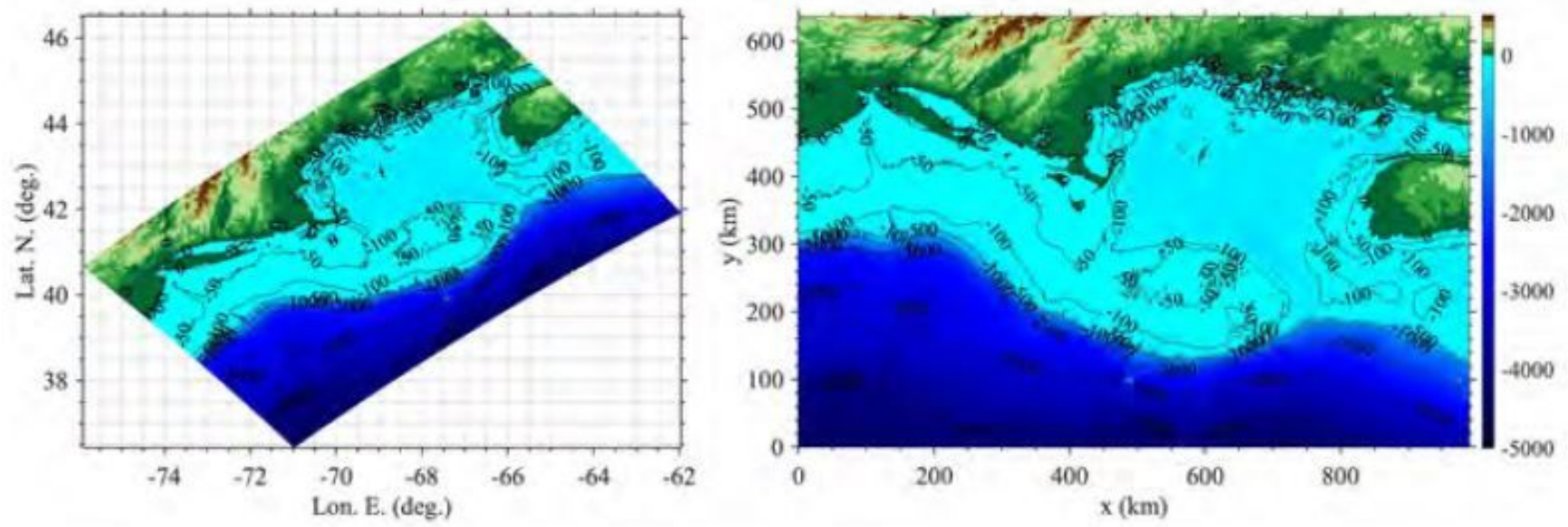

(b)
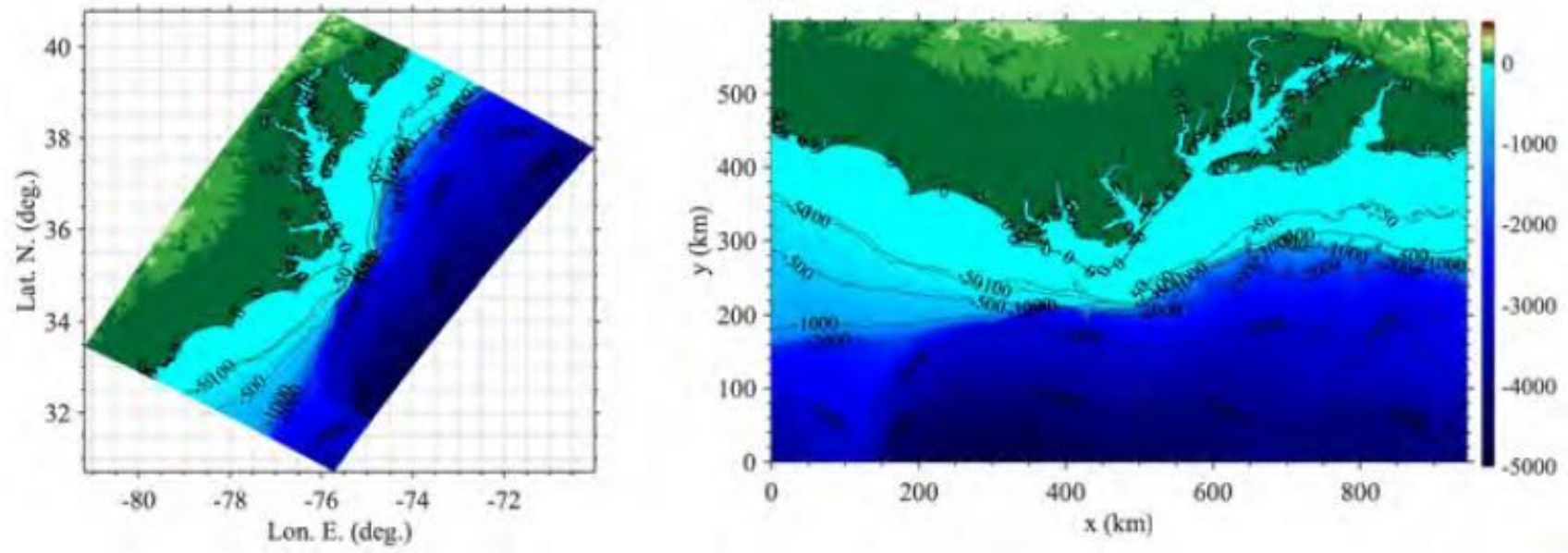

(c)
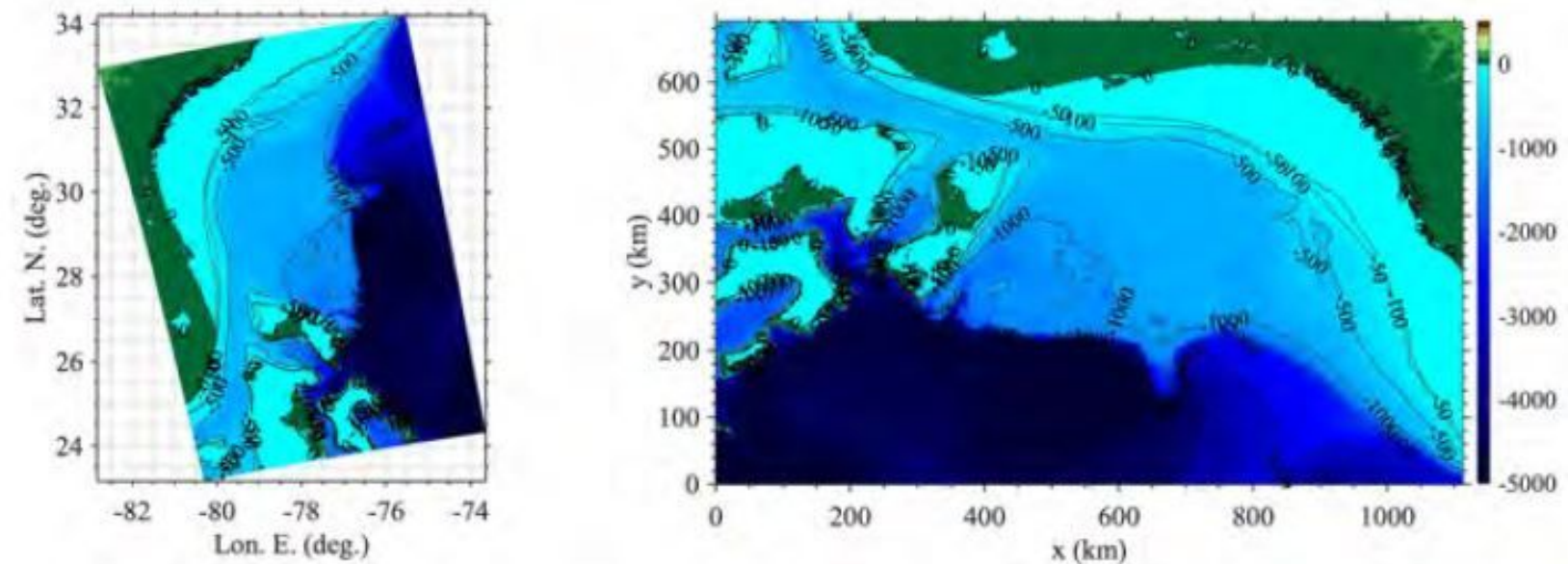

Figure 3

Footprints of $450 \mathrm{~m}$ resolution grids used in FUNWAVE, in spherical (left) and Cartesian (right) coordinates (Table 4, Fig. 1): (a) G1, (b) G2, (c) G3; color scales and contours are bathymetry/topography in meter. Note: The designations employed and the presentation of the material on this map do not imply the expression of any opinion whatsoever on the part of Research Square concerning the legal status of 
any country, territory, city or area or of its authorities, or concerning the delimitation of its frontiers or boundaries. This map has been provided by the authors.

(a)

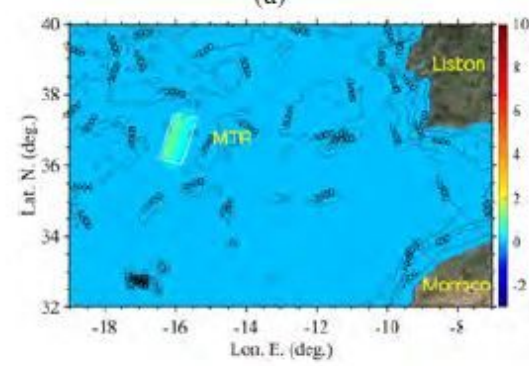

(c)

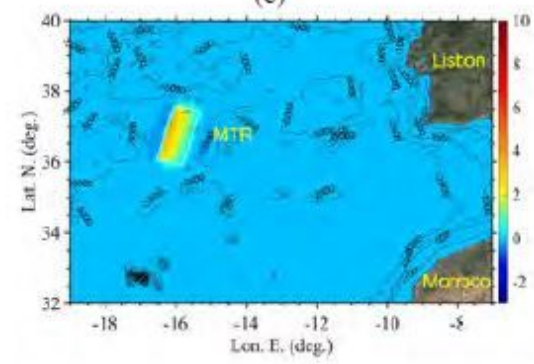

(e)

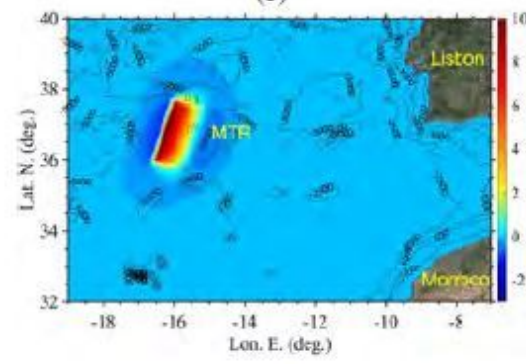

(g)

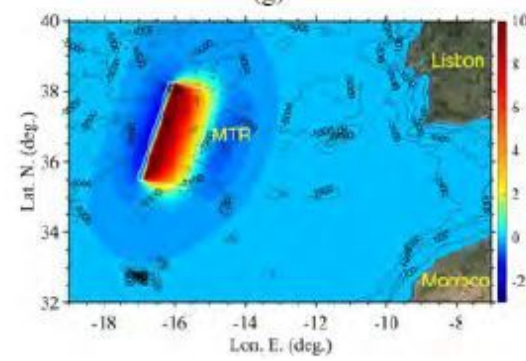

(i)

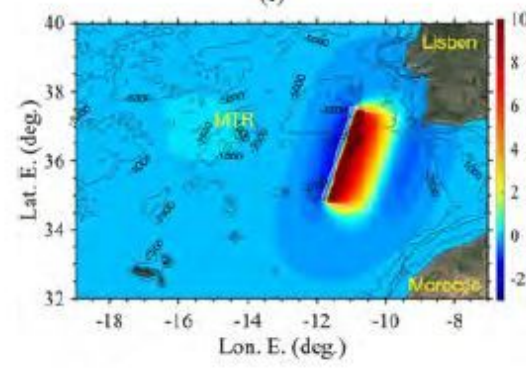

(b)

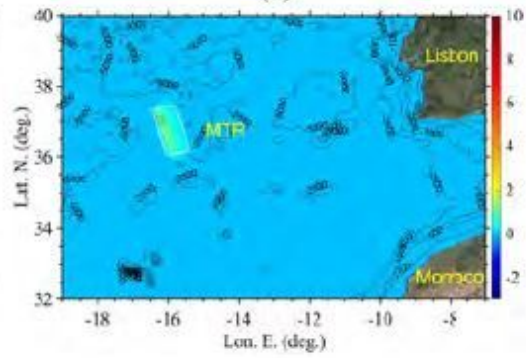

(d)

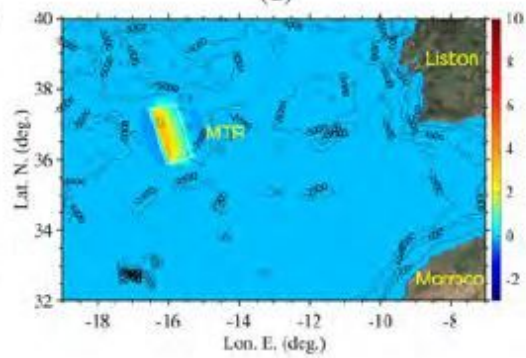

(f)

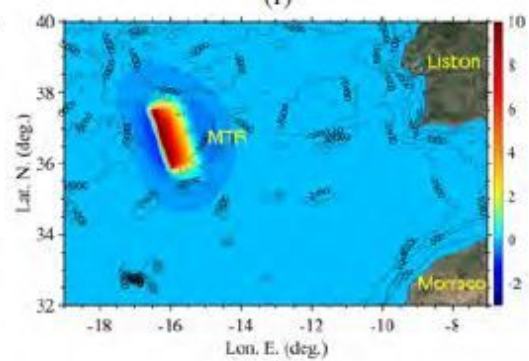

(h)

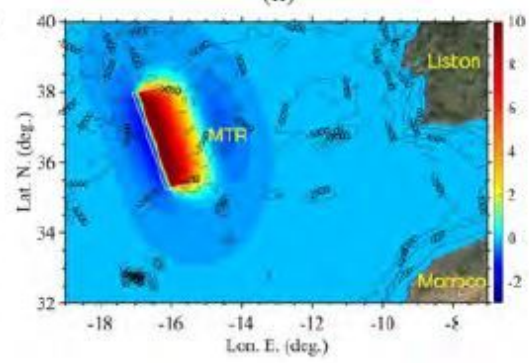

(j)

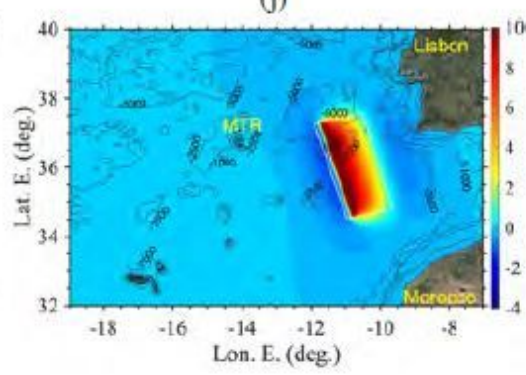

Figure 4

Initial surface elevations computed with Okada's [43] method (color scale in meter) for coseismic tsunami sources in the ACZ/LSB area (Fig. 1; Table 1) : (a) M8.0-MTR1; (b) M8.0-MTR2; (c) M8.3-MTR1; (d) M8.3MTR2; (e) M8.7-MTR1; (f) M8.7-MTR2; (g) M9.0-MTR1; (h) M9.0-MTR2; (i) M9.0-HSP1; (j) M9.0-HSP2. 
Each source has a single fault plane with footprint marked by white solid lines. Note, the M9.0 sources represent Lisbon 1755 proxies, located in either the ACZ, west of the MTR ("MTR"), or Horseshoe Plain ("HSP") areas (Fig. 1.)

(a)

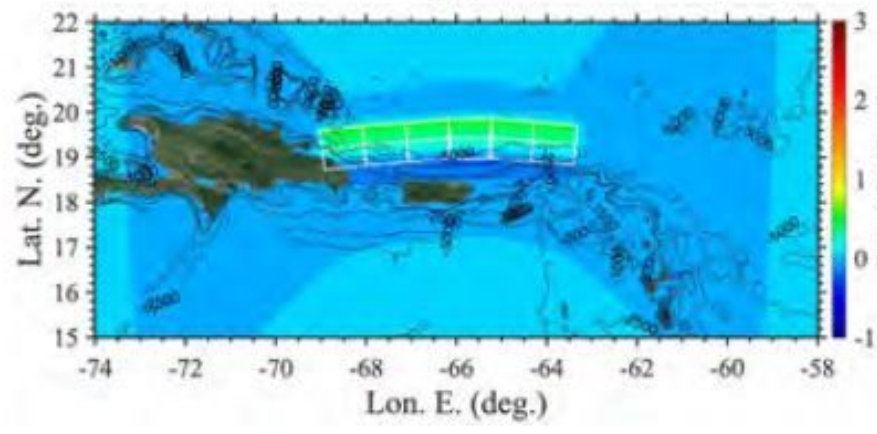

(c)

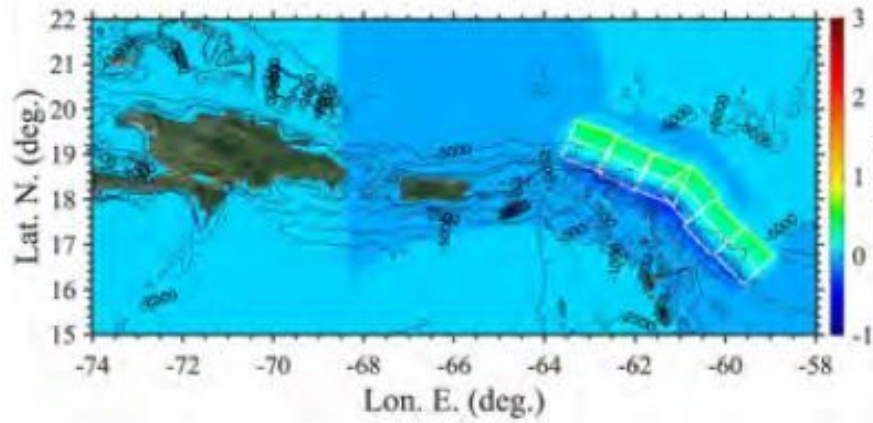

(e)

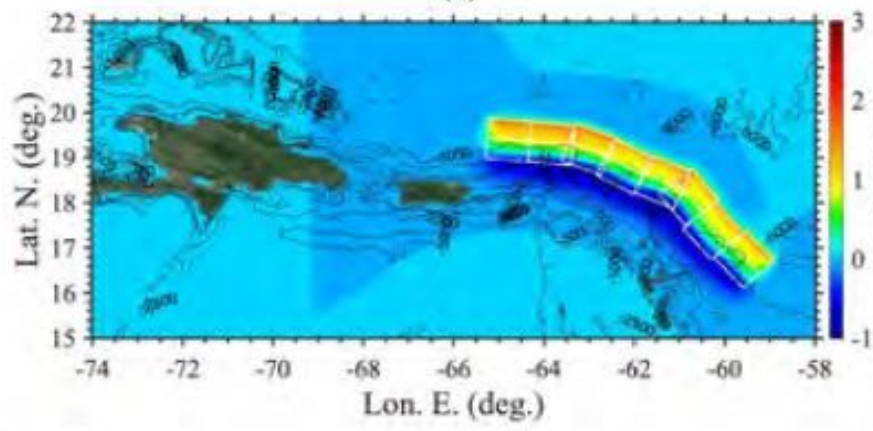

$(\mathrm{g})$

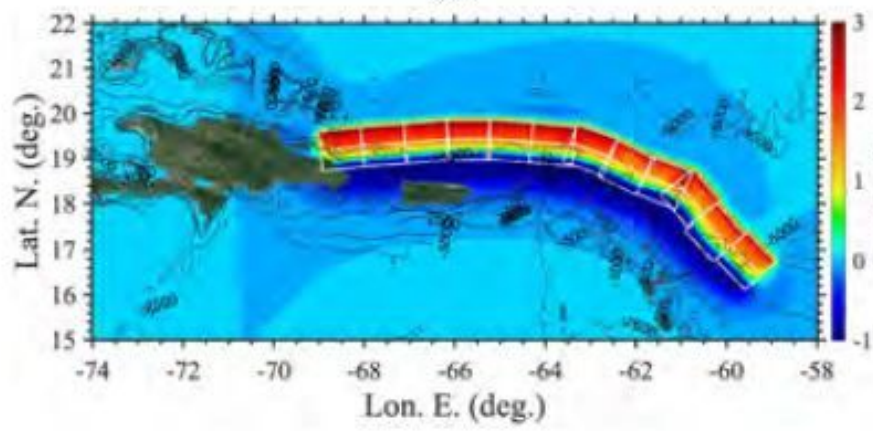

(b)

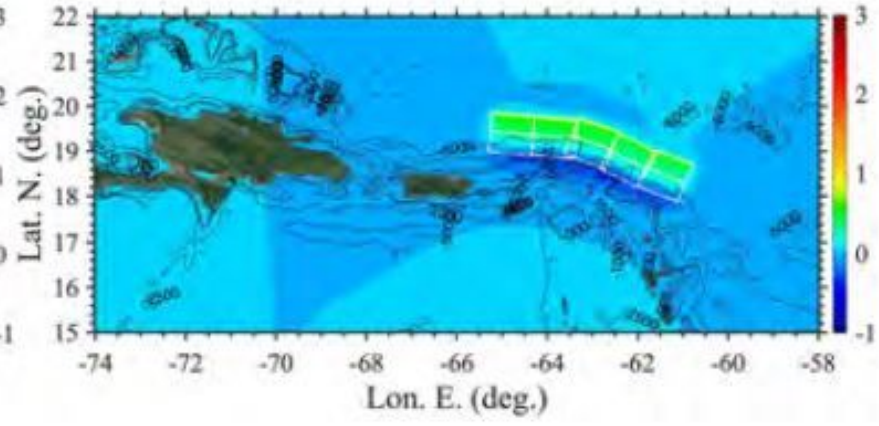

(d)

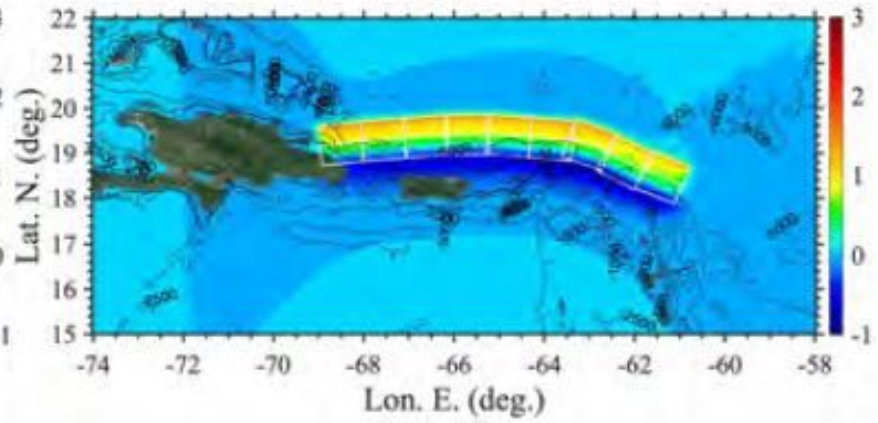

(f)

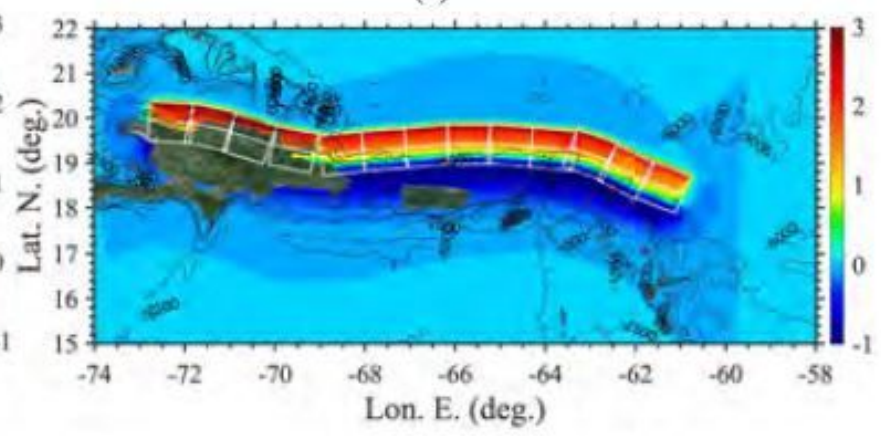

(h)

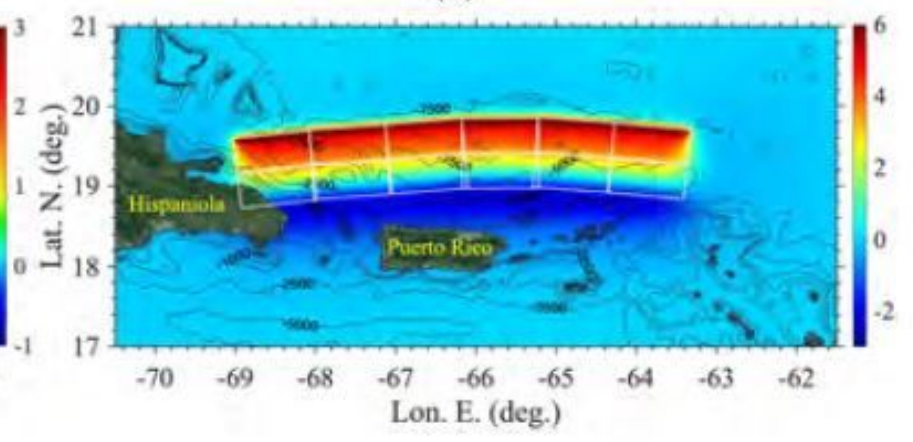

Figure 5

Initial surface elevations computed with Okada's [43] method for the modeled PRT/Caribbean arc tsunami sources (Fig. 1; Table 3): (a) M8.3-PRT1; (b) M8.3-PRT2; (c) M8.3-PRT3; (d) M8.7-PRT1; (e) M8.7- 
PRT2; (f) M9.0-PRT1; (g) M9.0-PRT2; and (h) M9.0-PRT3. All source parameters are given Tables 2 and 3. Color scale (in meter), with same scale is used in plots (b-h), for comparison. Note: The designations employed and the presentation of the material on this map do not imply the expression of any opinion whatsoever on the part of Research Square concerning the legal status of any country, territory, city or area or of its authorities, or concerning the delimitation of its frontiers or boundaries. This map has been provided by the authors.

(a)

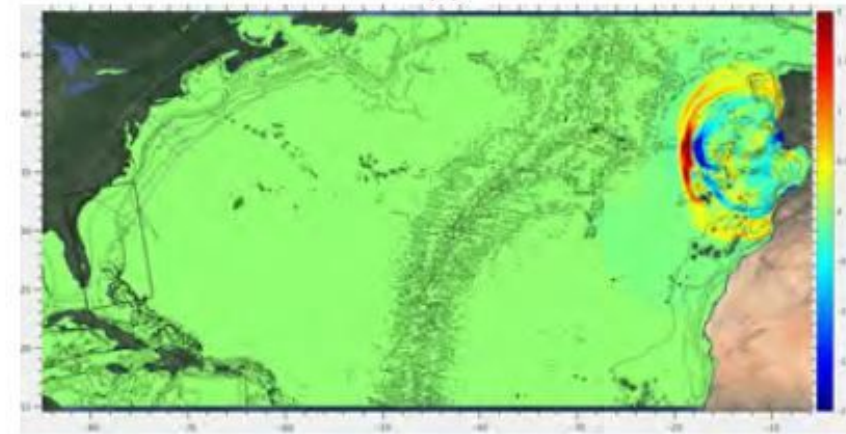

(c)

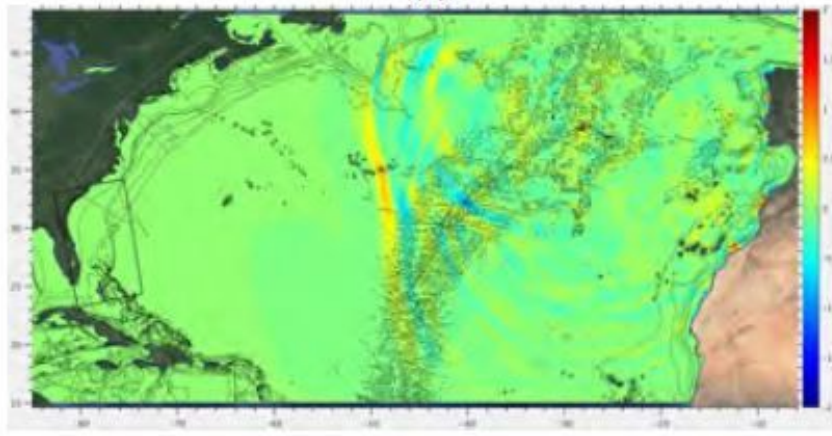

(e)

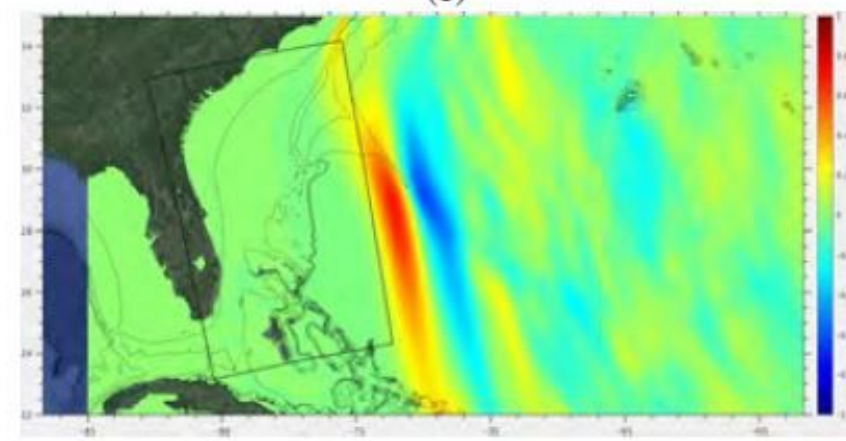

(g)

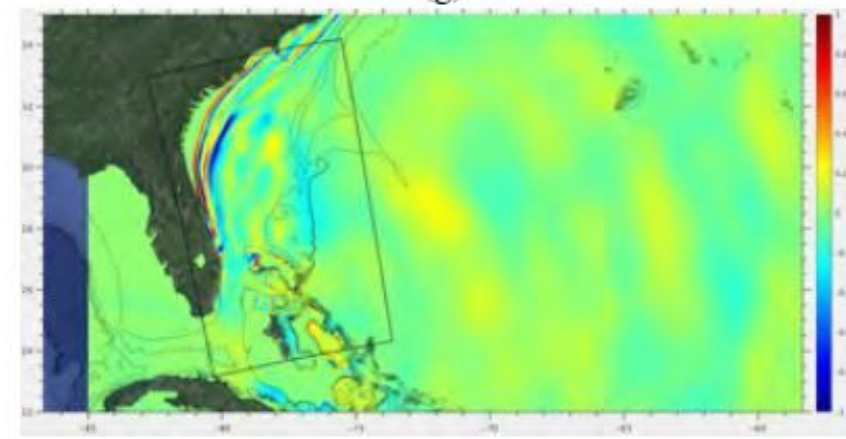

(b)

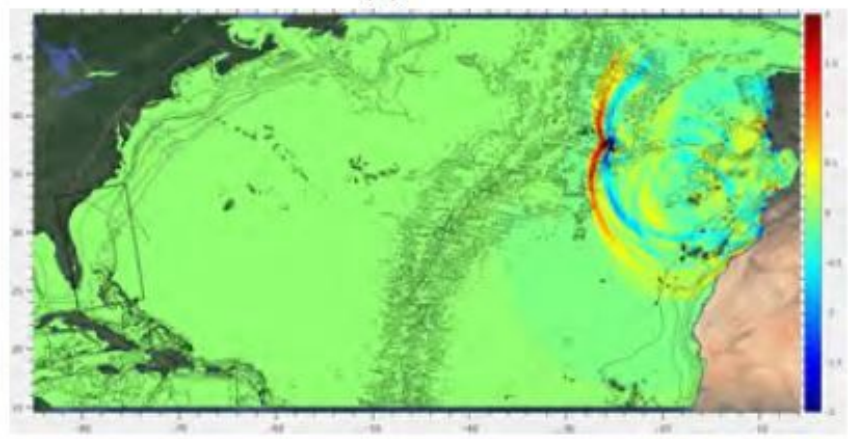

(d)

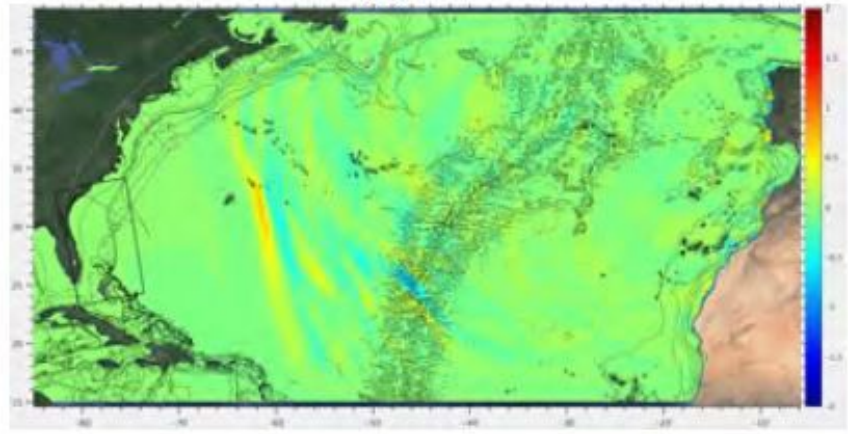

(f)

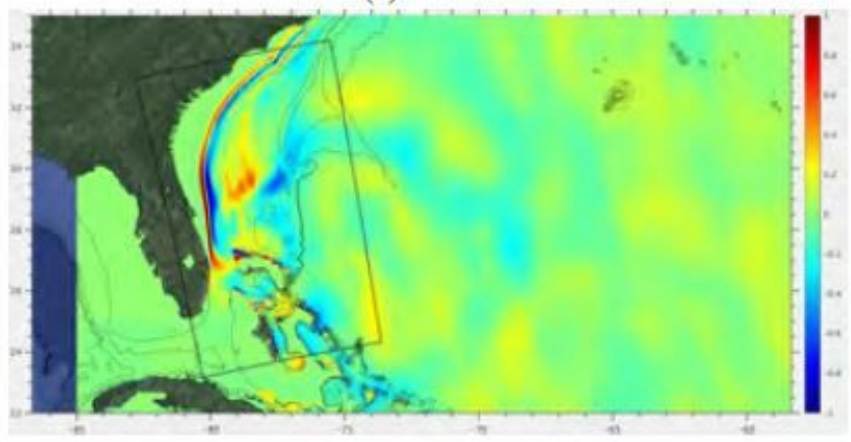

(h)

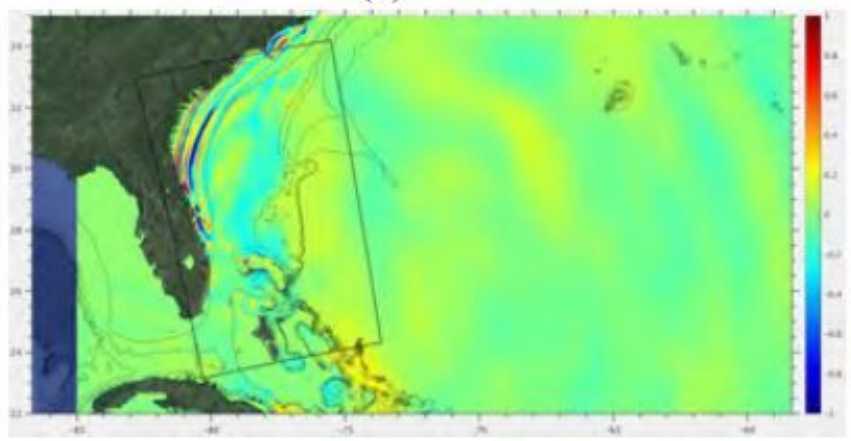


Snapshots of surface elevations (color scale in meter) computed for the Lisbon 1755 proxy source M9HSP1 (Fig. 4i; Table 1), at t = (a) 1, (b) 2, (c) 5, (d) 6.5, (e) 8, (f) 9.5, (g) 10.5, and (h) 11 h. Some isobaths are plotted for reference, but without labels to simplify the figures. Higher resolutions results are used wherever available. Note: The designations employed and the presentation of the material on this map do not imply the expression of any opinion whatsoever on the part of Research Square concerning the legal status of any country, territory, city or area or of its authorities, or concerning the delimitation of its frontiers or boundaries. This map has been provided by the authors.

(a)

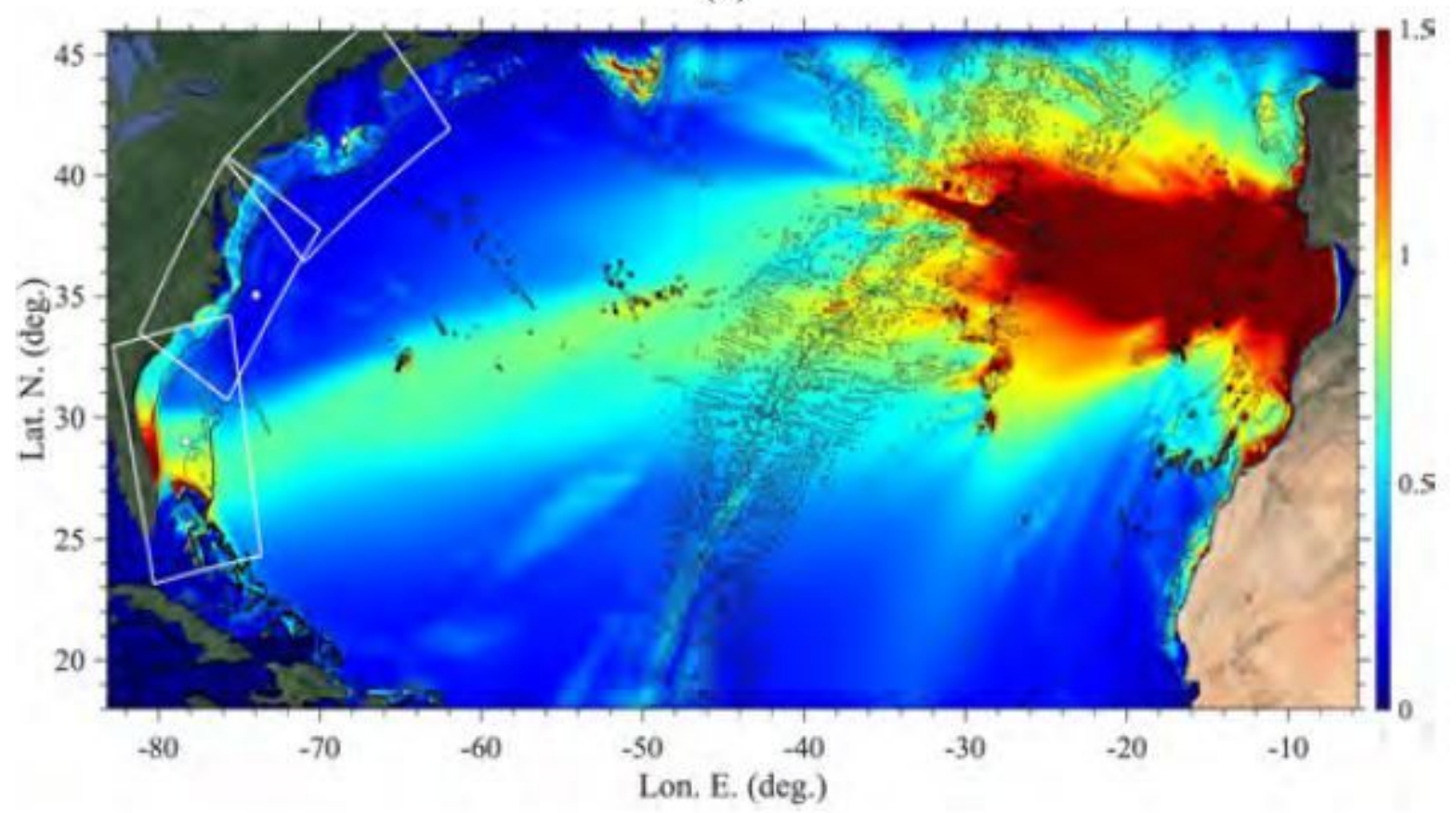

(b)

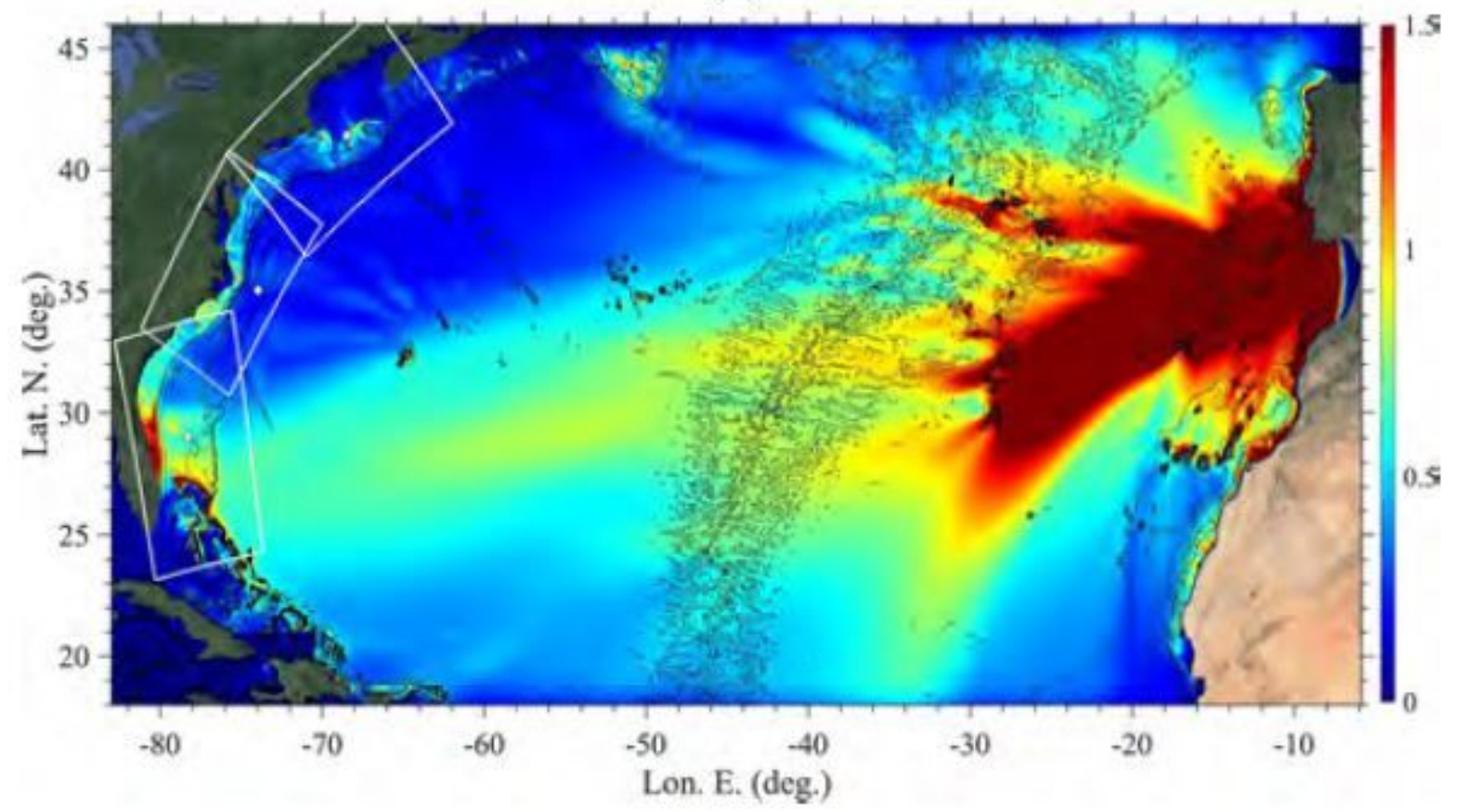

Figure 7 
Envelope of maximum surface elevation computed with FUNWAVE during 24h of simulations in grid Large G0 (Table 4; Fig. 1) for the: (a) M9-HSP1, and (b) M9-HSP2, historical Lisbon 1755 coseismic sources (with parameters listed in Table 1 and initial surface elevations in Figs. 4i,j). Color scales are surface elevation in meter.

(a)

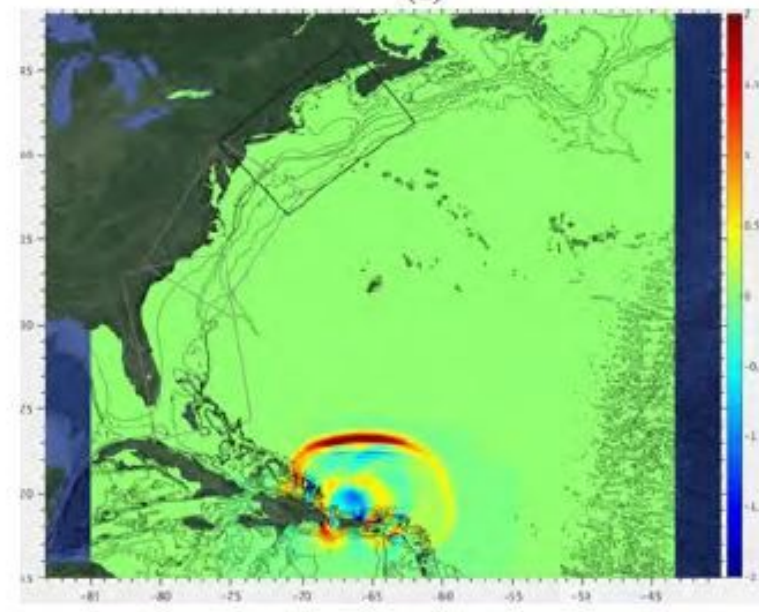

(c)

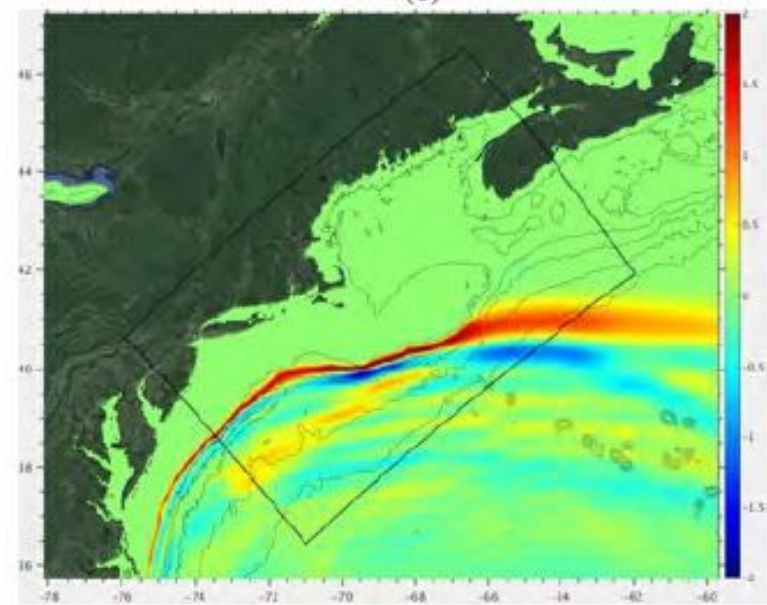

(e)

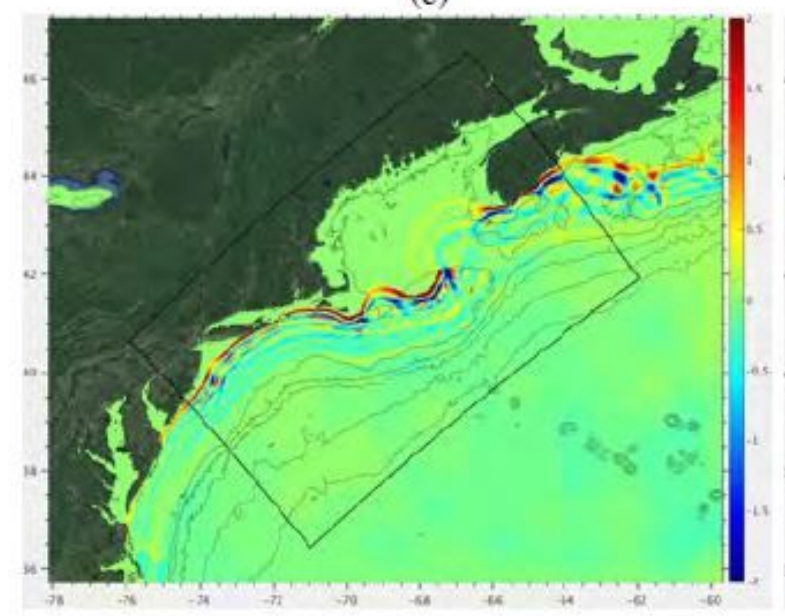

(b)

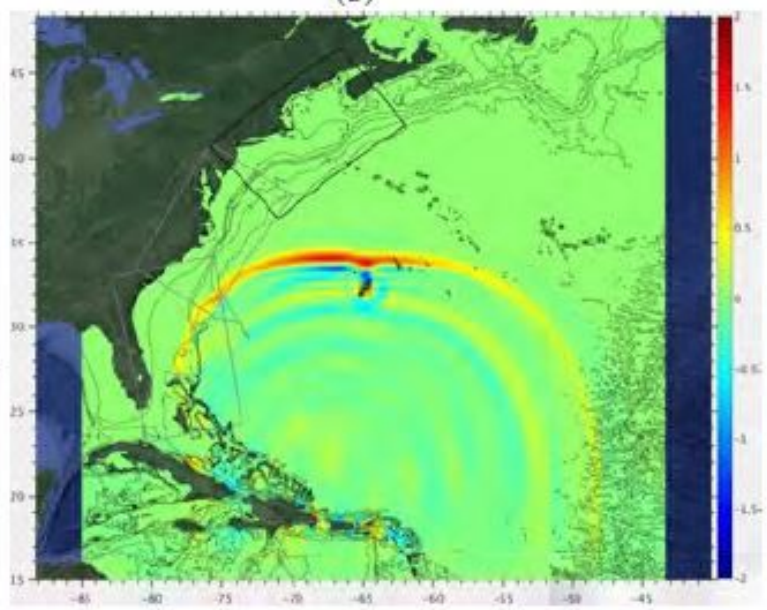

(d)

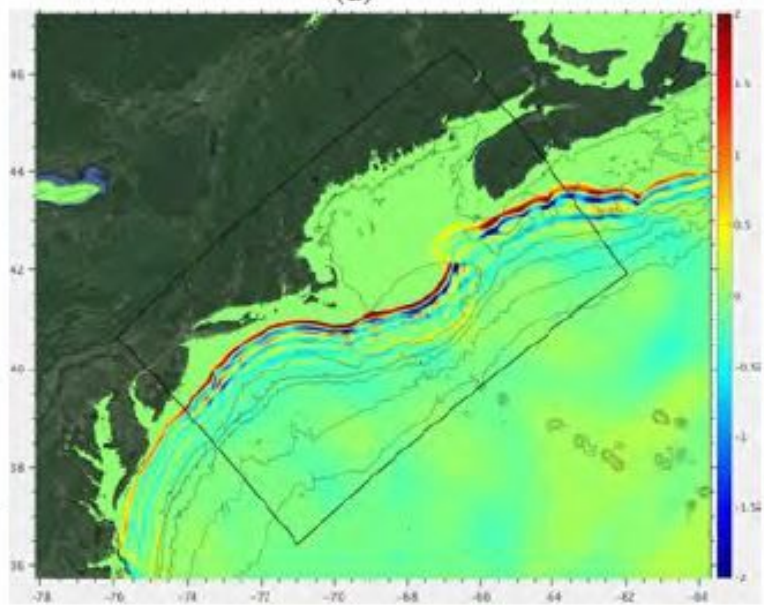

(f)

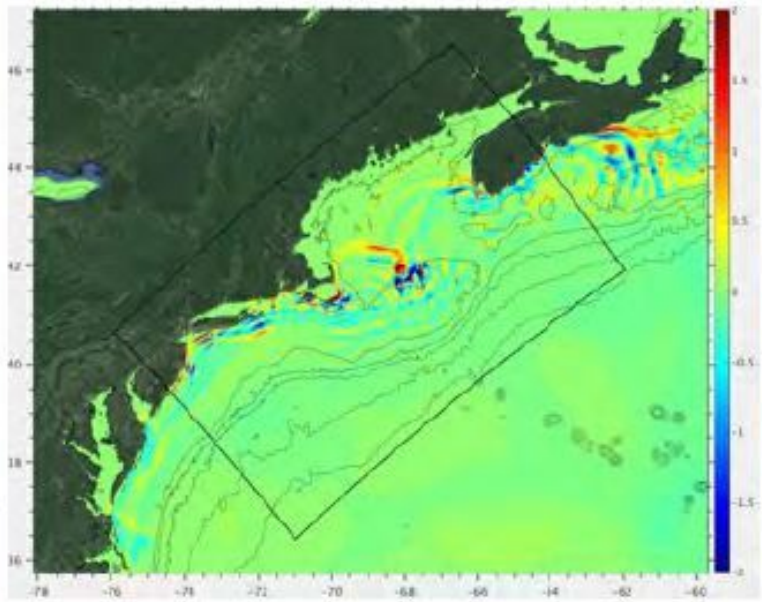

Figure 8 
Snapshots of surface elevations (color scale in meter) for the PRT/Caribbean arc source M9-PRT3 (Table 3; Fig. 5h), at t = (a) 0.5, (b) 2, (c) 3, (d) 4, (e) 4.5, and (f) $5 \mathrm{~h}$. Some isobaths are plotted for reference, but without labels to simplify the figures. Higher resolutions results are used wherever available. Note: The designations employed and the presentation of the material on this map do not imply the expression of any opinion whatsoever on the part of Research Square concerning the legal status of any country, territory, city or area or of its authorities, or concerning the delimitation of its frontiers or boundaries. This map has been provided by the authors.

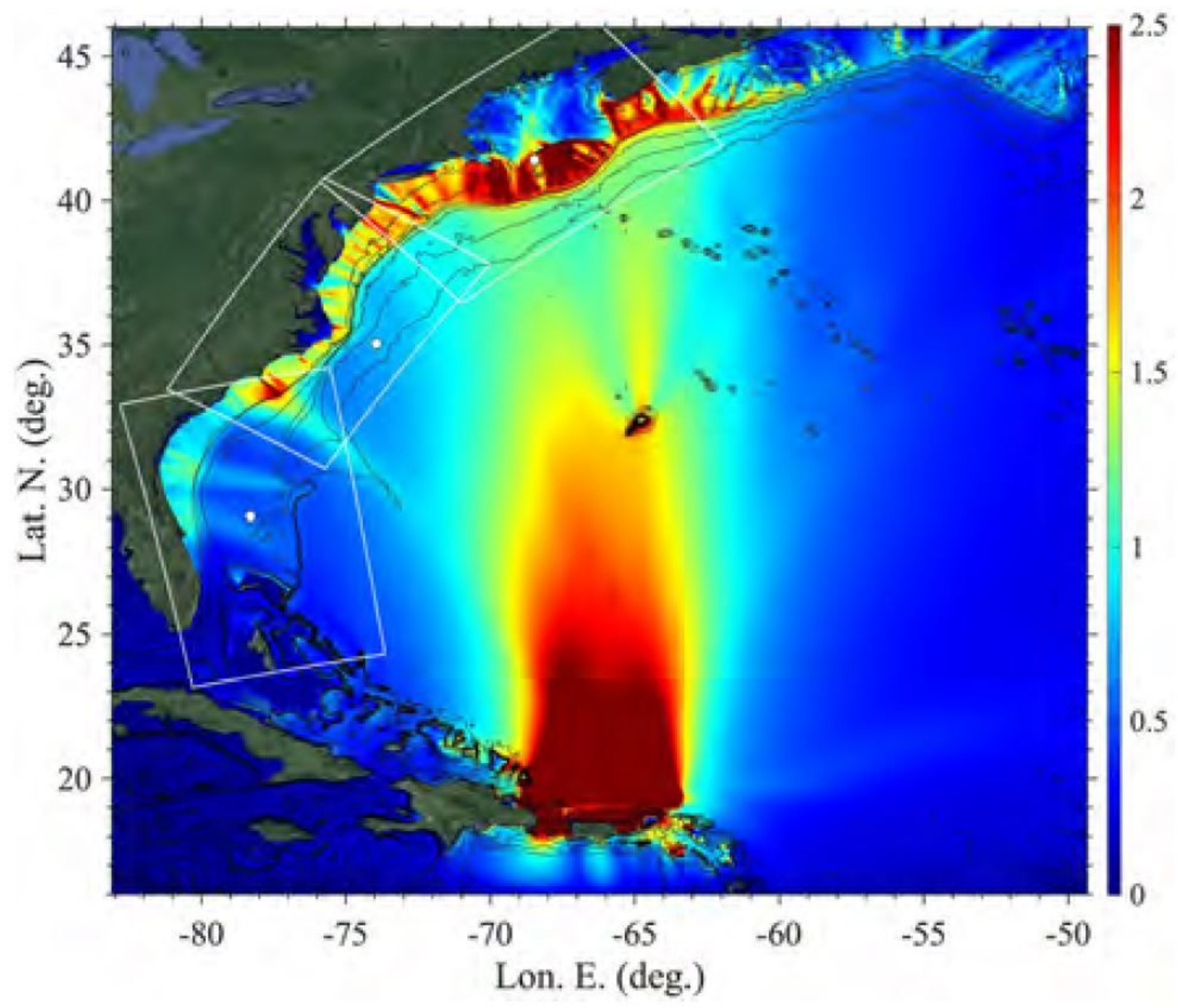

\section{Figure 9}

Envelope of maximum surface elevation computed with FUNWAVE during 24h of simulations in grid Local G0 (Table 4; Fig. 1) for the M9-PRT3 coseismic source (with parameters listed in Table 3 and initial surface elevations in Figs. 5h). Color scales are surface elevation in meter. Note: The designations employed and the presentation of the material on this map do not imply the expression of any opinion whatsoever on the part of Research Square concerning the legal status of any country, territory, city or 
area or of its authorities, or concerning the delimitation of its frontiers or boundaries. This map has been provided by the authors.
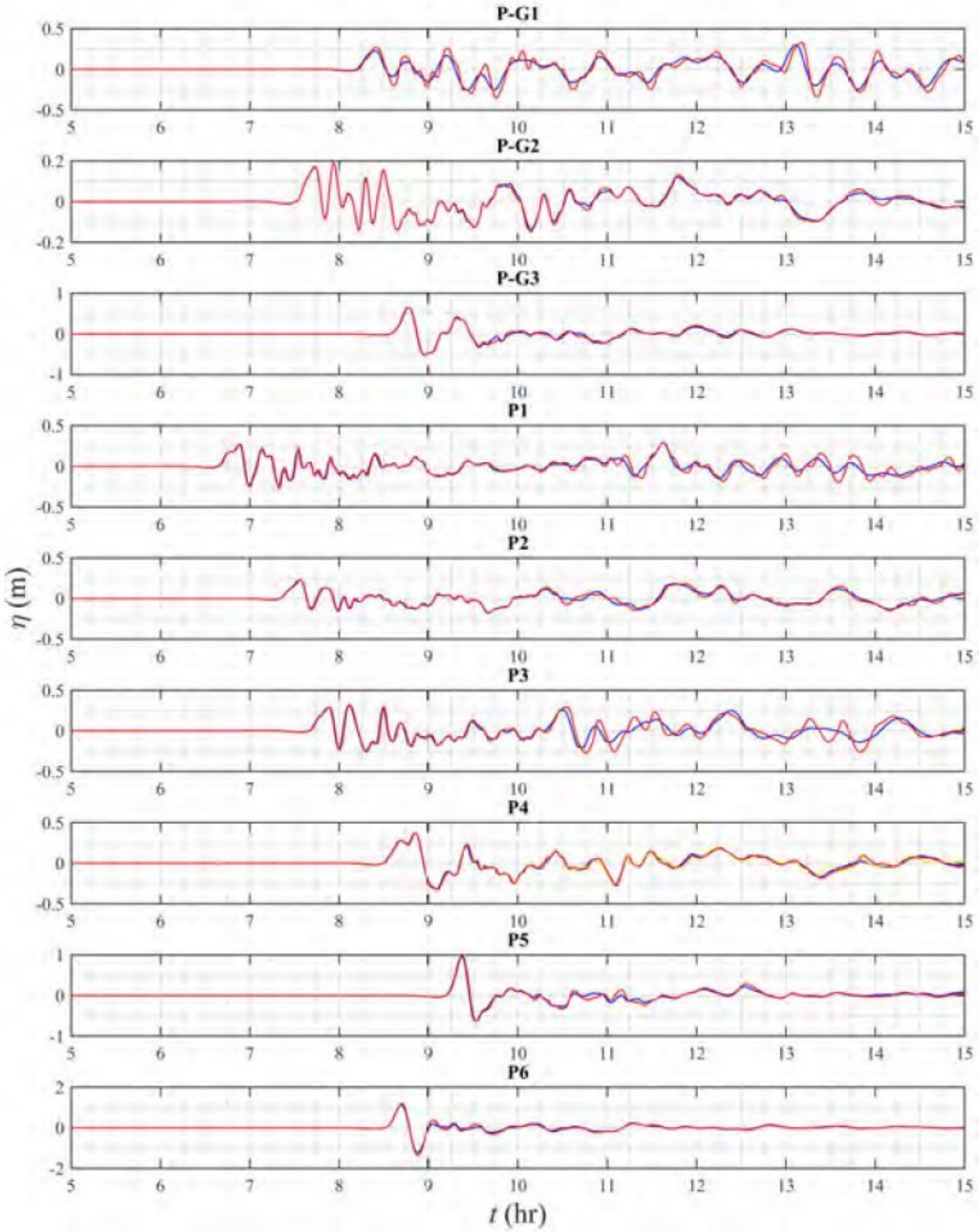

\section{Figure 10}

Time series of surface elevations computed for the M9-HSP1 source (Table 1), at the 9 save points defined in Table 5 (shown in Figs. 1): (blue lines) in grid Large G0; (red lines) in nested grids G1-G3. For 
point P4, yellow indicates surface elevation computed in G2, red indicates surface elevation computed in G3.
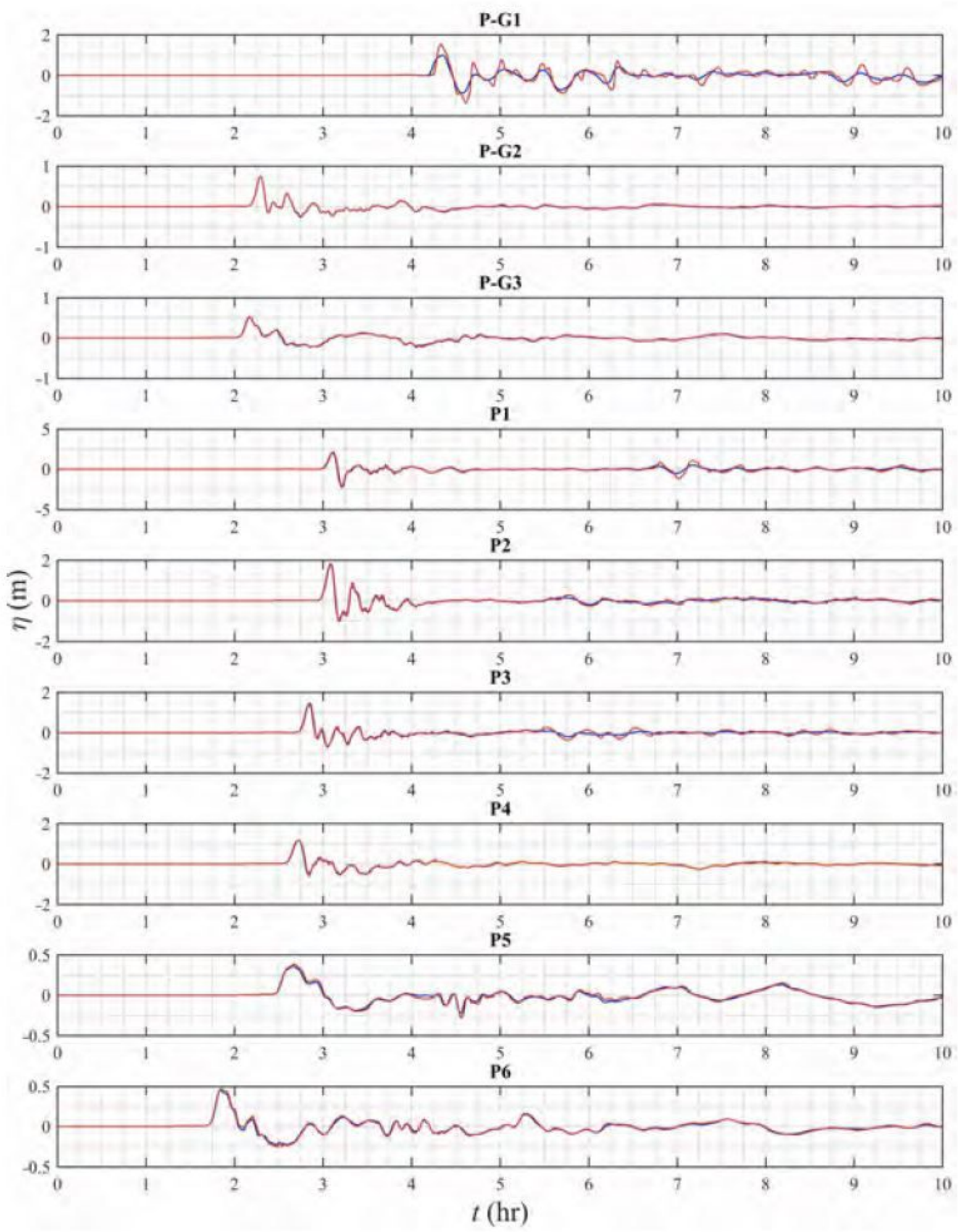

\section{Figure 11}

Time series of surface elevations computed for the M9-PRT3 source (Table 3), at the 9 save points defined in Table 5 (shown in Figs. 1): (blue lines) in grid Local G0; (red lines) in nested grids G1-G3. For 
point P4, black indicates surface elevation computed in G2, red indicates surface elevation computed in G3.

(a)

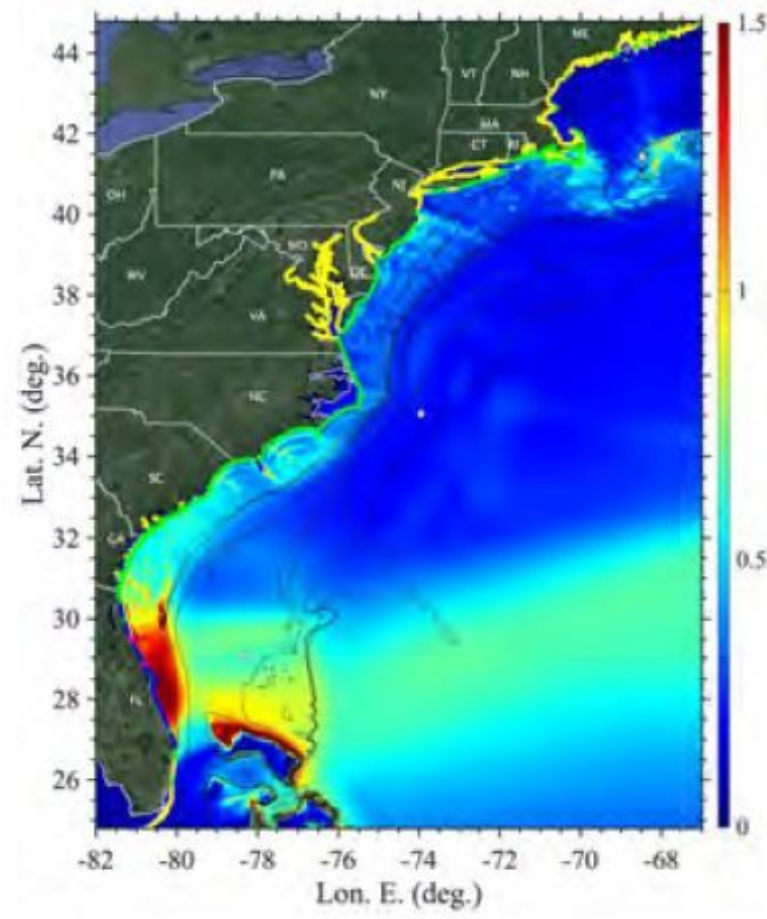

(b)

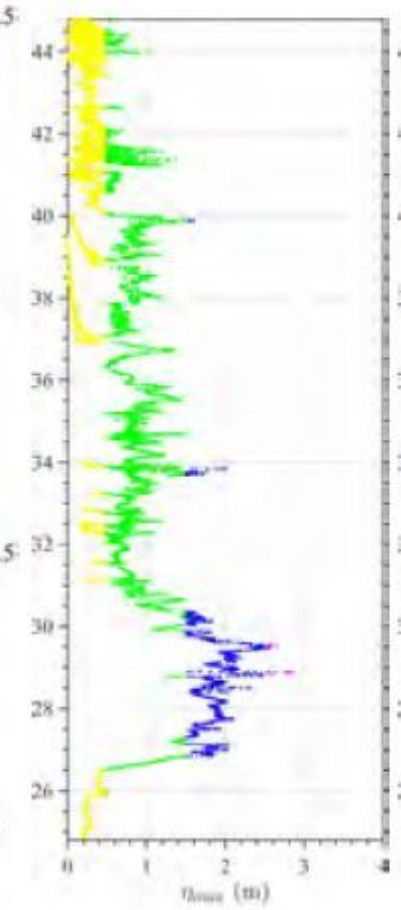

(c)

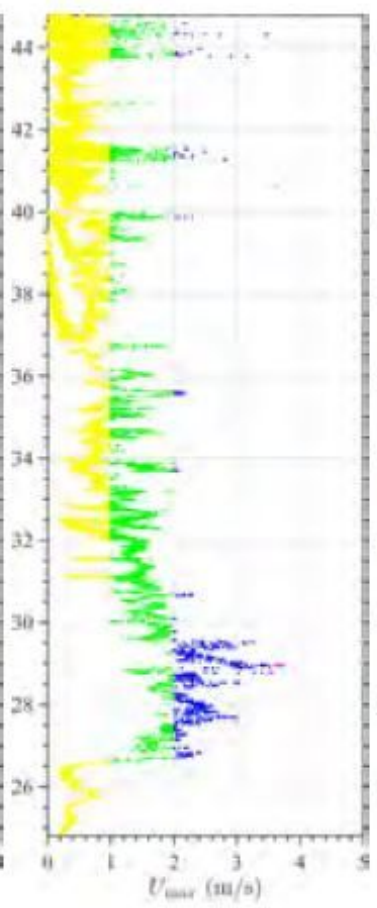

(d)

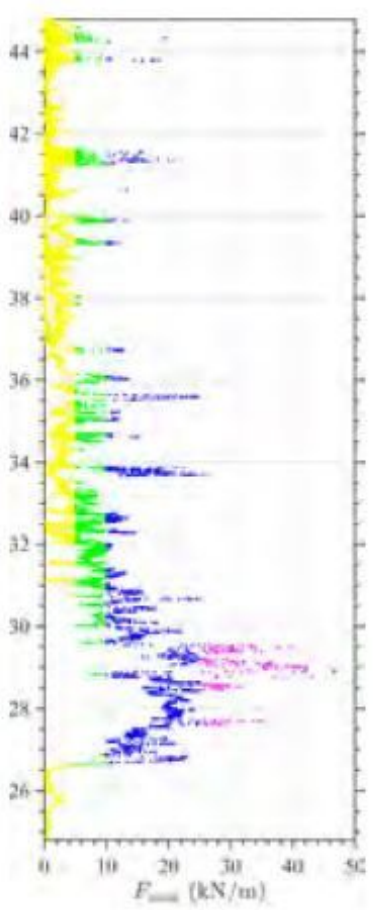

Figure 12

Hazard metrics along the USEC for the M9.0-HSP1 coseismic source (Table 1; Fig. 4i): (a) envelope of maximum surface elevations $₫$ max over grids G1,G2,G3 (color scale in meter; colored dots along the coast correspond to hazard intensity $\mathrm{C} 1 \mathrm{k}$ from $(\mathrm{b}))$; (b,c,d) color coded hazard intensity classes Cik ( $\mathrm{k}=1$; :..:; 5; yellow, green, blue, magenta and red; Table 6) with corresponding values of: $(i=1) \unrhd \max ,(i=2)$ Umax, and ( $\mathrm{i}=3$ ) Fmax, respectively, in the same grids; $\mathrm{J}=28$; 401 save points are defined along the $5 \mathrm{~m}$ isobath, here including large bays. Note: The designations employed and the presentation of the material on this map do not imply the expression of any opinion whatsoever on the part of Research Square concerning the legal status of any country, territory, city or area or of its authorities, or concerning the delimitation of its frontiers or boundaries. This map has been provided by the authors. 
(a)

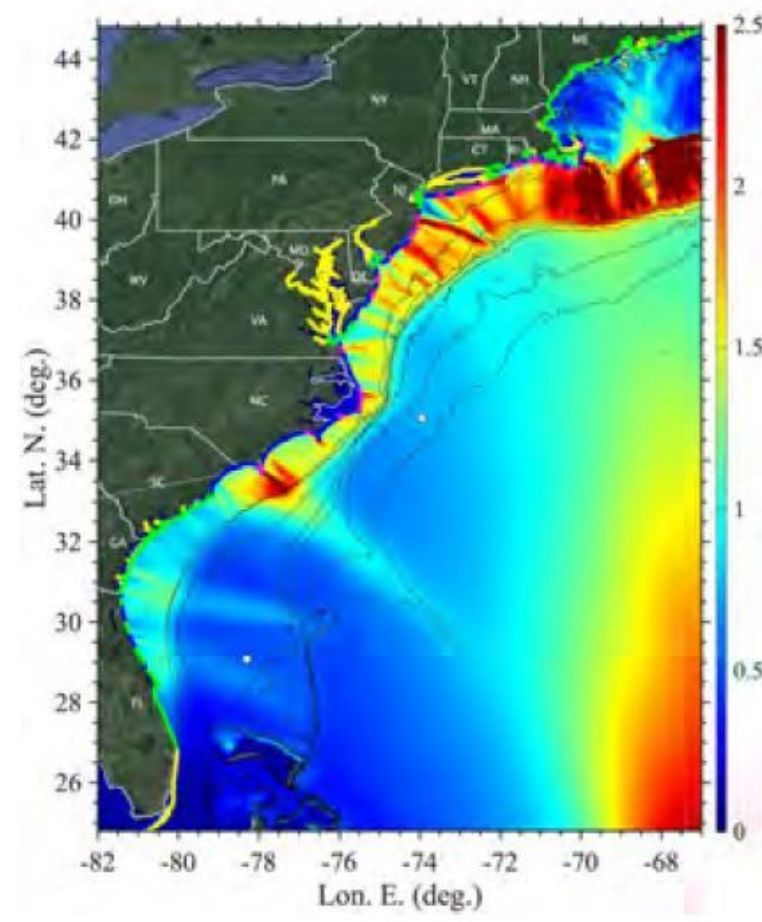

(b)

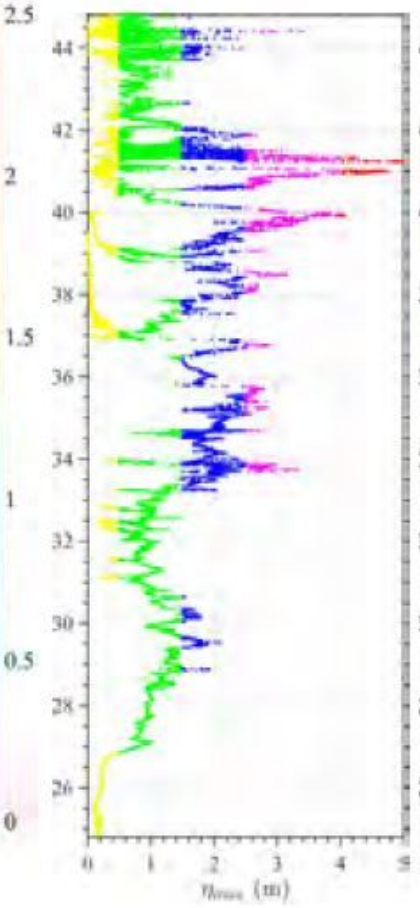

(c)

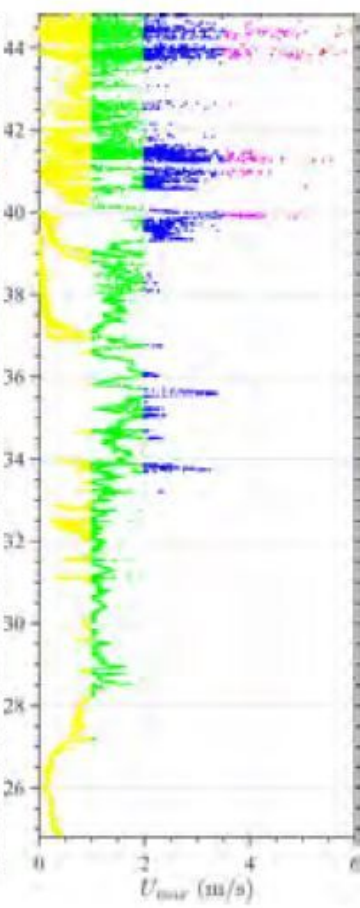

(d)

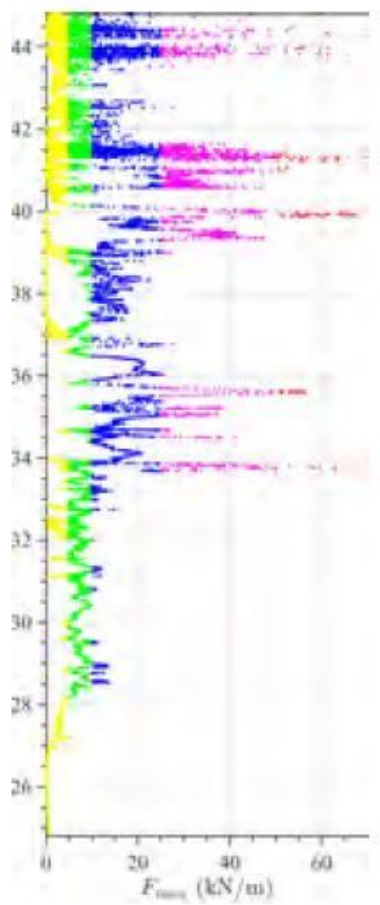

\section{Figure 13}

Same results as in Fig. 12, for the M9.0-PRT3 coseismic source (Table 3; Fig. 5h). Note: The designations employed and the presentation of the material on this map do not imply the expression of any opinion whatsoever on the part of Research Square concerning the legal status of any country, territory, city or area or of its authorities, or concerning the delimitation of its frontiers or boundaries. This map has been provided by the authors. 
(a)

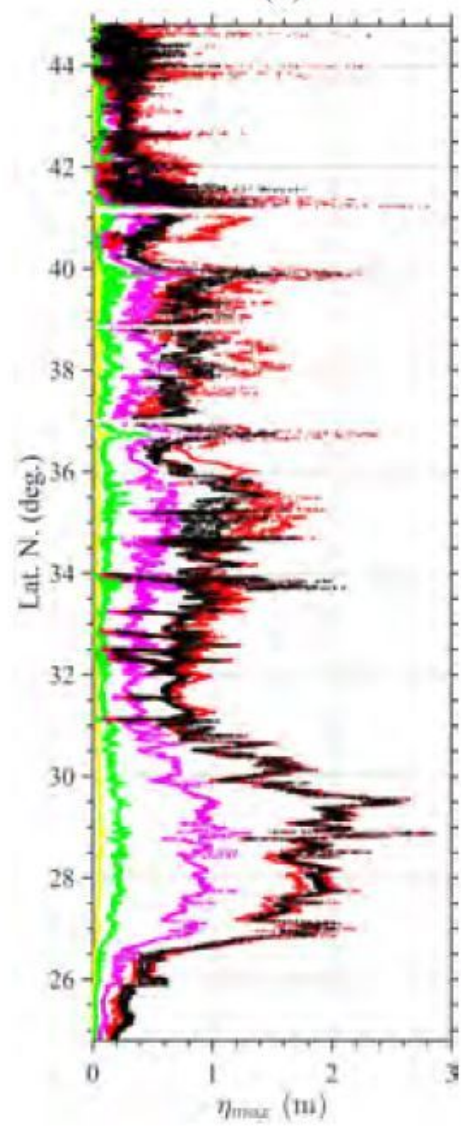

(b)

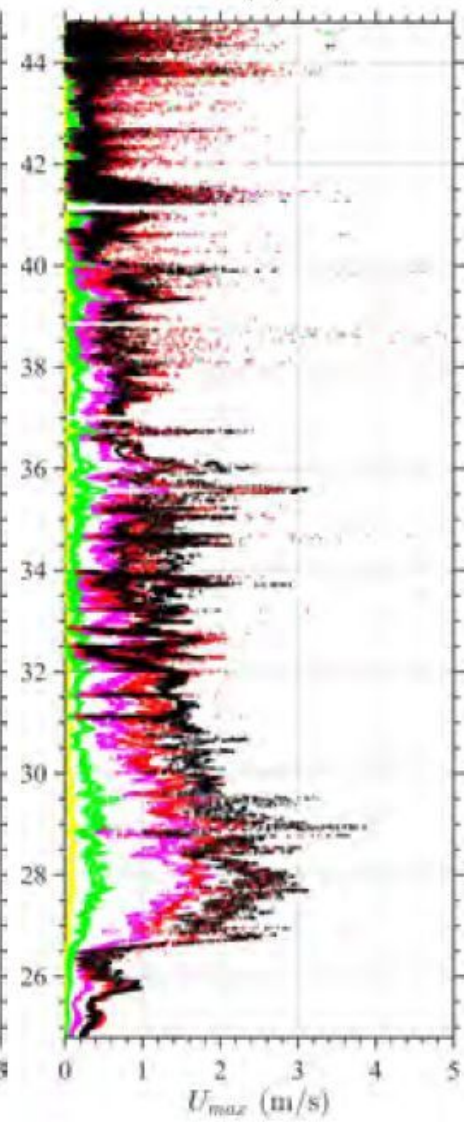

(c)

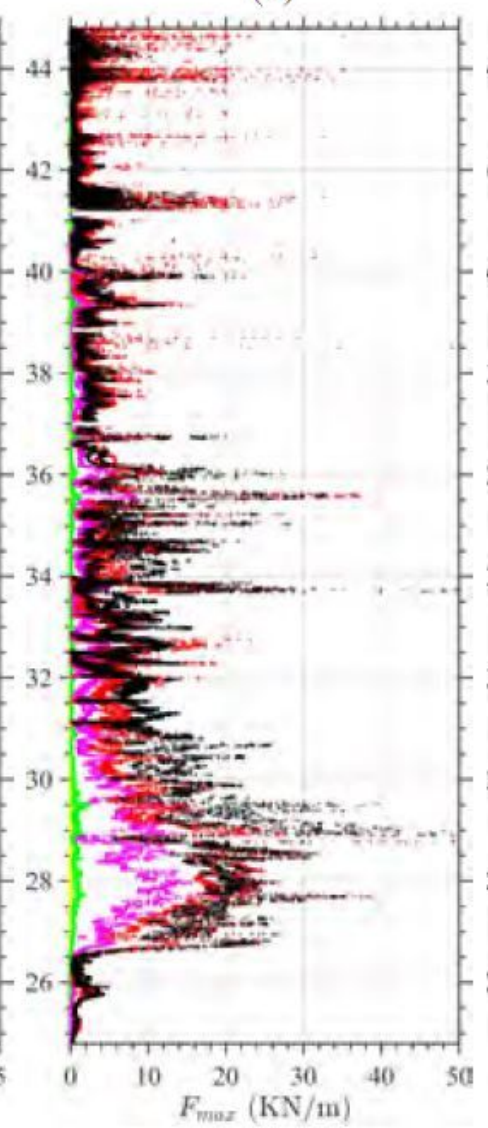

(d)

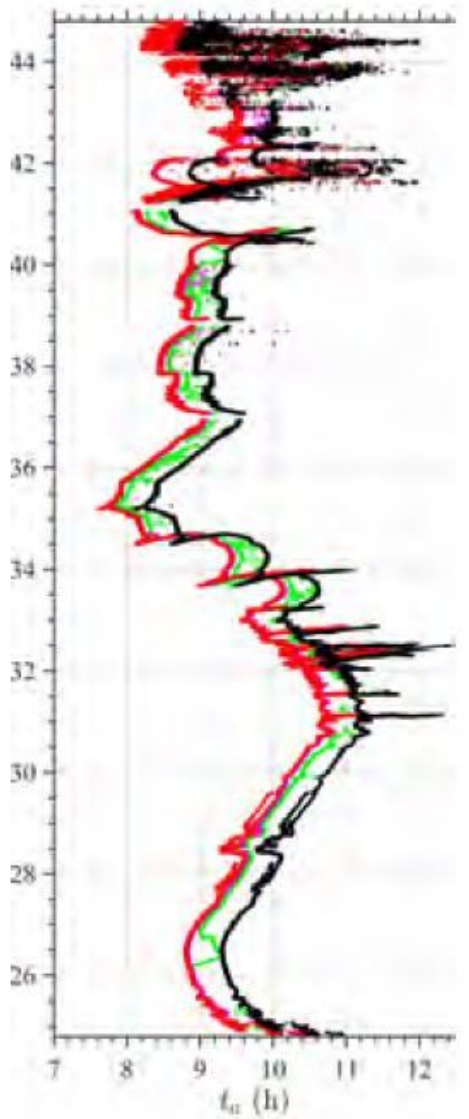

Figure 14

Envelope at the $5 \mathrm{~m}$ isobath ( $\mathrm{J}=18 ; 201$ save points excluding large bays) of maximum tsunami: (a) elevation; (b) current; (c) momentum force; and (d) arrival time, computed in grids G1,G2,G3, for the ten ACZ/LSB sources (Table 1; Fig. 4): (green) M8.0-MTR1, M8.0-MTR2; (yellow) M8.3-MTR1, M8.3- MTR2; (magenta) M8.7-MTR1, M8.7-MTR2; (red) M9.0-MTR1, M9.0-MTR2; and (black) M9.0-HSP1, M9.0-HSP2. Note, arrival times for the latter two sources, which are slightly longer, are not shown for clarity. 
(a)

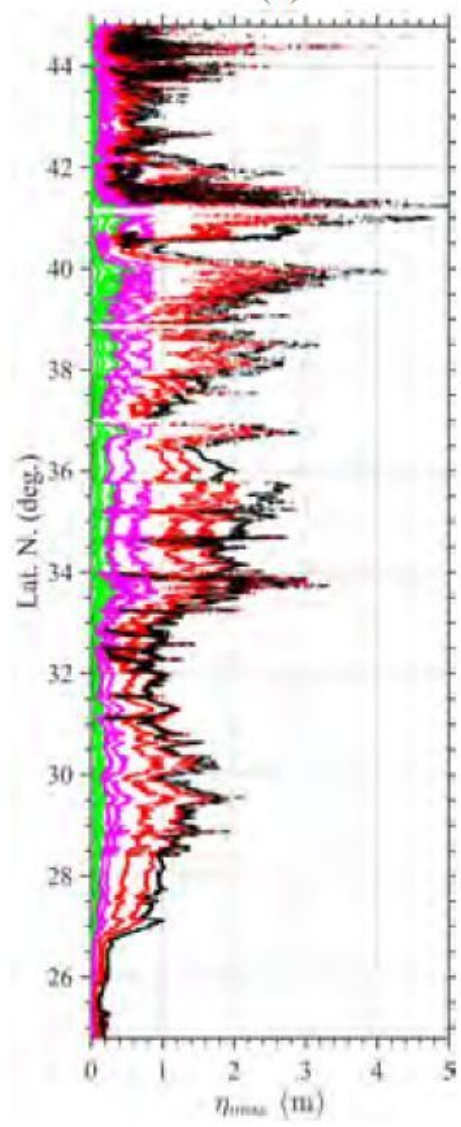

(b)

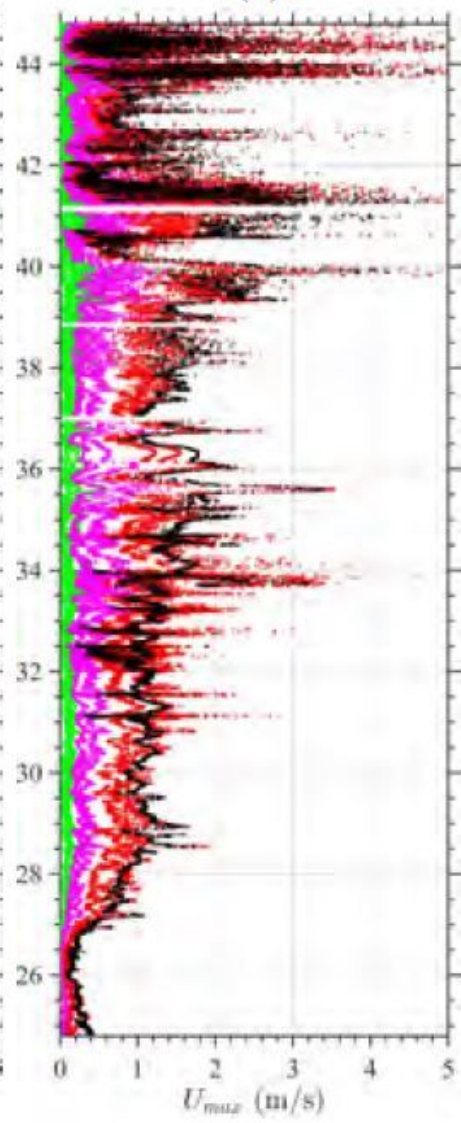

(c)

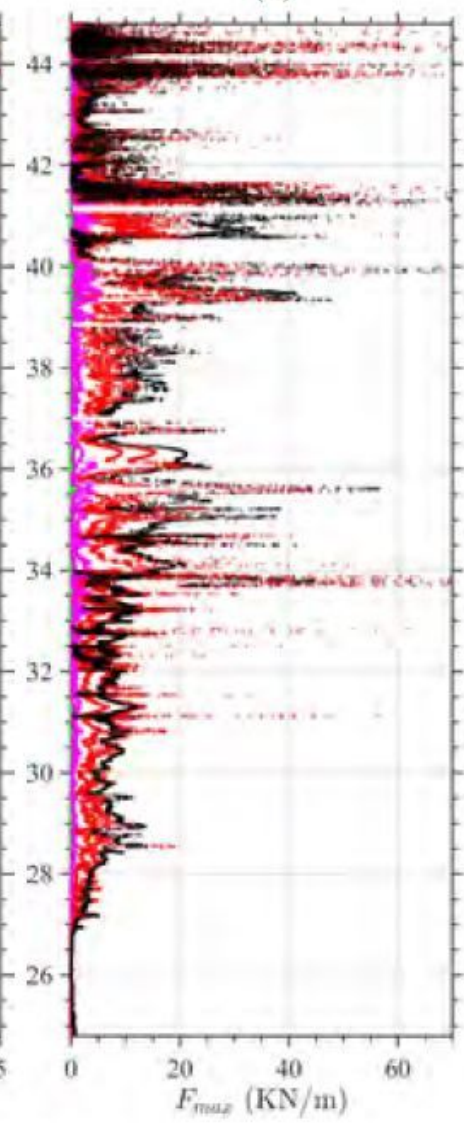

(d)

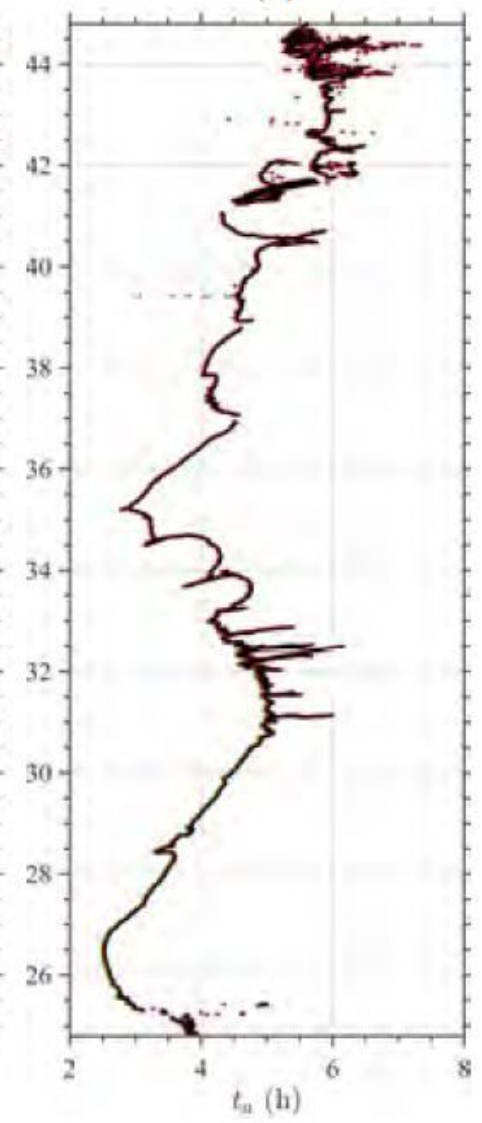

\section{Figure 15}

Envelope at the $5 \mathrm{~m}$ isobath ( $\mathrm{J}=18 ; 201$ save points excluding large bays) of maximum tsunami: (a) elevation; (b) current; (c) momentum force; and (d) arrival time, computed in grids $G 1, G 2, G 3$, for the eight PRT/Caribbean arc sources (Table 2.2; Fig. 5): (green) M8.3-PRT1, M8.3-PRT2, M8.3-PRT3; (magenta) M8.7-PRT1, M8.7-PRT2; (red) M9.0-PRT2, M9.0-PRT2; and (black) M9.0-PRT3. Note, arrival times for the latter source, which is similar, is not shown for clarity. 


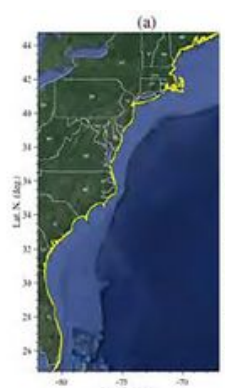

tencision

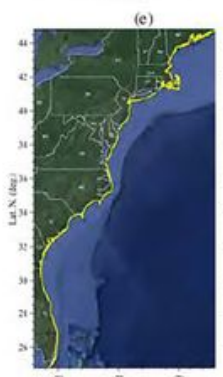

Linitiva

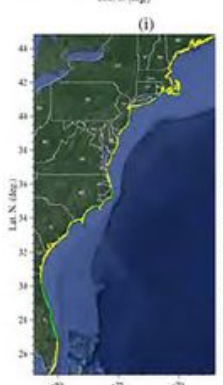

Le: 2 ives)

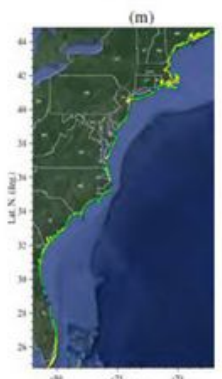

(ailian

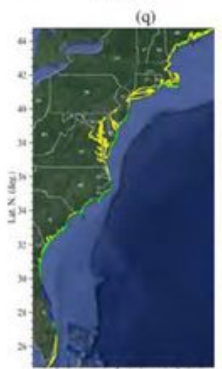

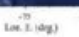

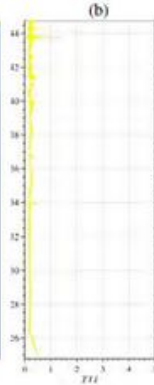

(f)

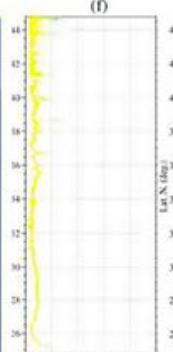

(j)

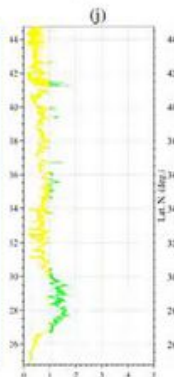

(n)
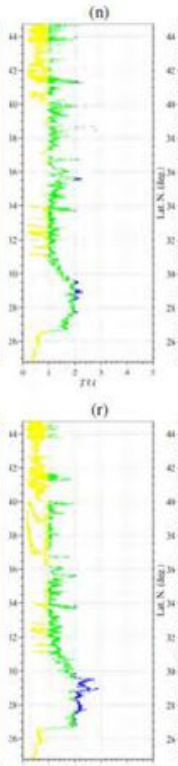

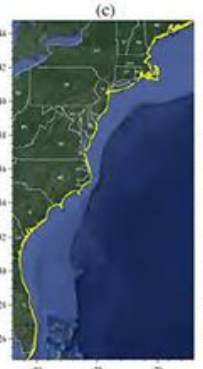

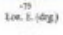

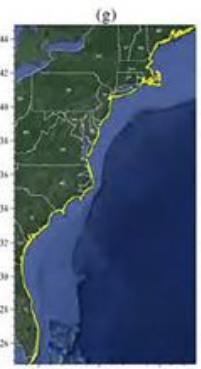

$\tan 2(x)$

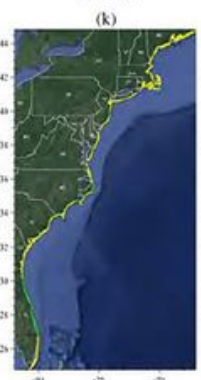

thetion

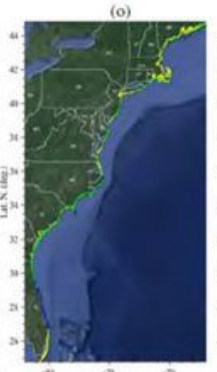

$\operatorname{lin} i \sin$

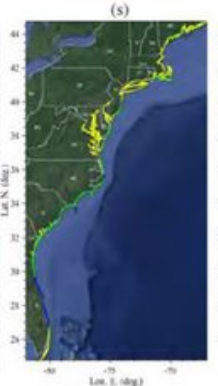

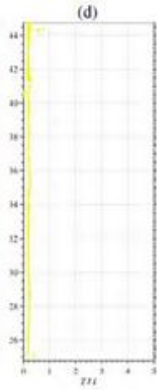
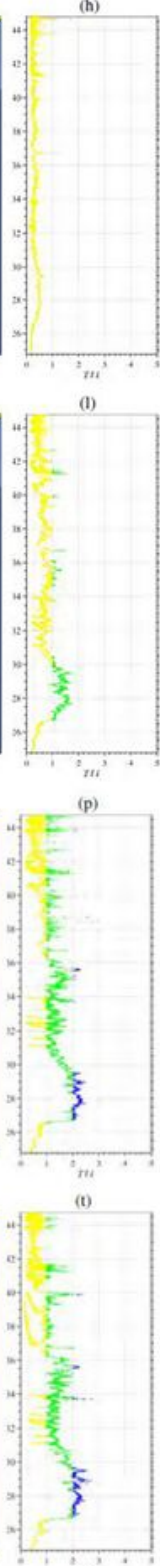

\section{Figure 16}

Tsunami intensity index computed for ten LSB/ACZ sources (Table 1; Fig. 4), based on results of the 4 hazard metrics in Fig. 14: (a,b) M8.0-MTR1; (c,d) M8.0-MTR2; (e,f) M8.3-MTR1; (g,h) M8.3-MTR2; (i,j) M8.7-MTR1; (k,l) M8.7-MTR2; (m,n) M9.0-MTR1; (o,p) M9.0-MTR2; (q,r) M9.0-HSP1; (s,t) M9.0-HSP2. TII metric weights are wi $=[0.40,0.30,0.15,0.15]$, and color coded hazard intensity classes are: (yellow) low; (green) medium low; (blue) medium (magenta) medium high, (red) heigh, and (black) extreme. Note, for 
the last 2 sources, the TII is also provided within the large bays. Note: The designations employed and the presentation of the material on this map do not imply the expression of any opinion whatsoever on the part of Research Square concerning the legal status of any country, territory, city or area or of its authorities, or concerning the delimitation of its frontiers or boundaries. This map has been provided by the authors.
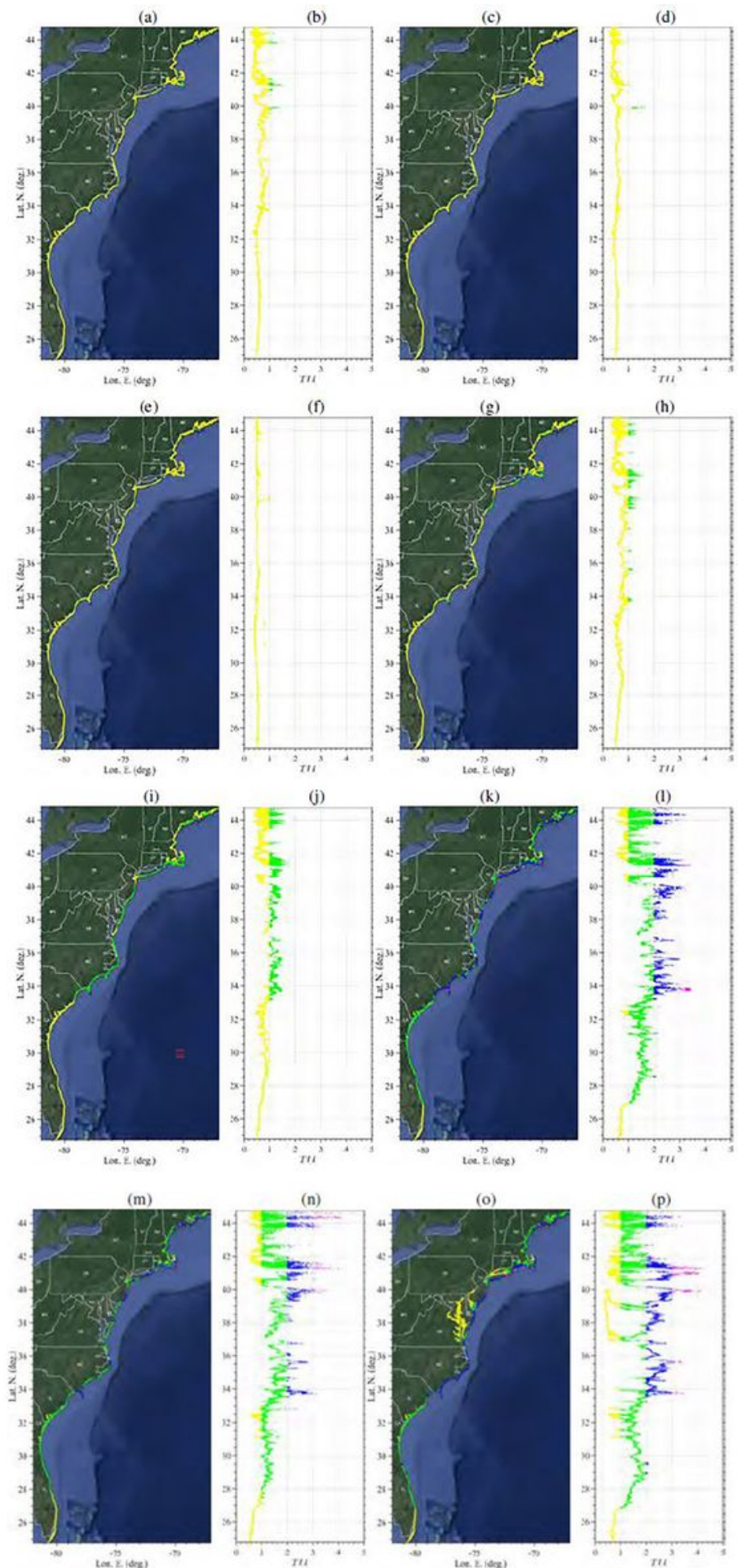

Figure 17 
Tsunami intensity index computed for eight PRT/Caribbean arc sources (Table 3; Fig. 5), based on results of the 4 hazard metrics in Fig. 15: (a,b) M8.3-PRT1; (c,d) M8.3-PRT2; (e,f) M8.3-PRT3; (g,h) M8.7- PRT1;

(i,j) M8.7-PRT2; $(\mathrm{k}, \mathrm{l})$ M9.0-PRT1; $(\mathrm{m}, \mathrm{n})$ M9.0-PRT2; $(0, \mathrm{p})$ M9.0-PRT3. TII metric weights are wi $=[0.40,0.30$, $0.15,0.15$ ], and color coded hazard intensity classes are: (yellow) low; (green) medium low; (blue) medium (magenta) medium high, (red) heigh, and (black) extreme. Note, for the last source, the TII is also provided within the large bays. Note: The designations employed and the presentation of the material on this map do not imply the expression of any opinion whatsoever on the part of Research Square concerning the legal status of any country, territory, city or area or of its authorities, or concerning the delimitation of its frontiers or boundaries. This map has been provided by the authors.

\section{Supplementary Files}

This is a list of supplementary files associated with this preprint. Click to download.

- HSP1.mp4

- PRT3.mp4 